University of Tennessee Health Science Center

UTHSC Digital Commons

\title{
$5-2019$
}

\section{Investigation of Cell-Type-Specific Effects and Synergistic Interactions Between Genes in Duplication 15q Syndrome}

Kevin A. Hope

University of Tennessee Health Science Center

Follow this and additional works at: https://dc.uthsc.edu/dissertations

Part of the Neurosciences Commons

\section{Recommended Citation}

Hope, Kevin A. (0000-0002-5722-5070), "Investigation of Cell-Type-Specific Effects and Synergistic Interactions Between Genes in Duplication 15q Syndrome" (2019). Theses and Dissertations (ETD). Paper 489. http://dx.doi.org/10.21007/etd.cghs.2019.0481.

This Dissertation is brought to you for free and open access by the College of Graduate Health Sciences at UTHSC Digital Commons. It has been accepted for inclusion in Theses and Dissertations (ETD) by an authorized administrator of UTHSC Digital Commons. For more information, please contact jwelch30@uthsc.edu. 


\title{
Investigation of Cell-Type-Specific Effects and Synergistic Interactions Between Genes in Duplication 15q Syndrome
}

\author{
Abstract \\ Duplication $15 q$ syndrome (Dup15q) is a genetic disorder caused by duplications of the 15q11.2-q13.1 \\ region and is characterized by developmental delay, autism spectrum disorder, and treatment resistant \\ epilepsy. Extra copies of the E3 ubiquitin ligase UBE3A and elevated levels of UBE3A expression in \\ neurons are thought to be the primary cause of Dup15q phenotypes. However, animal models \\ overexpressing UBE3A in neurons have not successfully recapitulated all aspects of Dup $15 q$ syndrome, \\ especially epilepsy. Here, we used Drosophila melanogaster (fruit flies) to investigate Dup $15 q$ syndrome. \\ In Chapter 2 we explored whether Dube3a, the Drosophila homolog of UBE3A, is imprinted in the fly brain. \\ In mammals, UBE3A undergoes complex imprinting and is expressed only from the maternal allele in \\ mature neurons. Prior to this work the imprinting status of Dube3a in flies was unclear. Here, we present \\ evidence that Dube3a is not imprinted and is biallelically expressed in the fly. Next, in Chapter 3 we \\ examined the interaction between Dube3a and HERC2. HERC2 is also an E3 ubiquitin ligase located in the \\ $15 q 11.2-q 13.1$ critical region and is duplicated in all Dup15q individuals. HERC2 physically interacts with \\ and stimulates the ubiquitin ligase activity of UBE3A in vitro. We found that Drosophila HERC2 appears to \\ stimulate the ubiquitin ligase function of Dube3a, and Dube3a and HERC2 interact synergistically to \\ impact phenotypes associated with Dup $15 q$ syndrome in vivo. Data presented in Chapter 3 suggests that \\ genes other than UBE3A are important in generating the Dup15q phenotypes and should not be ignored \\ when modeling Dup15q syndrome. In Chapter 4 we investigated how non-neuronal cells, specifically glial \\ cells, contribute to the seizure phenotype of Dup15q syndrome. We found that elevated levels of Dube3a \\ or UBE3A in glia causes seizures, while overexpression in neurons does not. These data are consistent \\ with mammalian models in which UBE3A elevation in neurons does not generate seizures. Furthermore, \\ overexpression of Dube3a in glia reduced protein levels of the Na+/K+ ATPase, ATP $\alpha$. ATP $\alpha$ down \\ regulation in glia is both necessary and sufficient to generate seizures. In Chapter 5 we further \\ characterized our Dup15q epilepsy model and investigated cell type specific effects of Dube3a \\ overexpression in glia compared to neurons using whole transcriptome and whole proteome analyses. We \\ found that elevation of Dube3a in glia caused a cell non-autonomous down regulation of synaptic \\ proteins in neurons while simultaneously causing a cell autonomous upregulation of glutathione S- \\ transferases (GSTs) in glial cells. Finally, we showed that the upregulation of GSTs is common to multiple \\ different Drosophila gliopathic seizure lines, not just our Dup15q epilepsy model. GSTs play a role in drug \\ metabolism, and elevation of these enzymes may underlie the treatment resistant nature of some \\ epilepsies including Dup15q syndrome. The results from these studies highlight the role that glial cell \\ dysfunction may play in generating seizures in Dup $15 q$ syndrome and could ultimately provide novel \\ avenues for epilepsy treatments.
}

\section{Document Type}

Dissertation

\section{Degree Name}

Doctor of Philosophy (PhD)

\section{Program}

Biomedical Sciences

Research Advisor

Lawrence T. Reiter, Ph.D. 


\section{Keywords}

Drosophila, Dube3a, Dup15q, Seizure, UBE3A

\section{Subject Categories}

Medicine and Health Sciences | Neurosciences 
Investigation of Cell-Type-Specific Effects and Synergistic Interactions Between Genes in Duplication 15q Syndrome

\author{
A Dissertation \\ Presented for \\ The Graduate Studies Council \\ The University of Tennessee \\ Health Science Center
}

\author{
In Partial Fulfillment \\ Of the Requirements for the Degree \\ Doctor of Philosophy \\ From The University of Tennessee
}

By

Kevin A. Hope

May 2019 
Chapter 4 (C) 2017 Elsevier Inc.

All other material (C) 2018 by Kevin A. Hope.

All rights reserved. 


\section{DEDICATION}

I dedicate this work to my parents, Michelle and Bob, and my wife, Francesca. 


\section{ACKNOWLEDGEMENTS}

Dr. Lawrence Reiter, thank you for your guidance throughout graduate school. You have taught me valuable lessons applicable to both inside and outside the lab.

The members of my committee, Dr. Janis O’Donnell, Dr. J. Paul Taylor, Dr. John Boughter, and Dr. Jim Sutcliffe, thank you for your support throughout this process. An additional thank you to Dr. O'Donnell and Dr. Sutcliffe for making the drive to Memphis for our meetings.

Thank you to Dr. Johnson at the Molecular Resource Center for assisting with the RNAseq data analysis and Dr. Lopez-Ferrer at ThermoFisher for assisting with the proteomics experiments.

The Duplication 15q Alliance, thank you for providing me with financial support over the past 4 years. I hope that I have given back to the Dup $15 \mathrm{q}$ community as much as you have given me.

Finally, thank you to all the flies for this work would not have been possible without them. 


\begin{abstract}
Duplication 15q syndrome (Dup15q) is a genetic disorder caused by duplications of the 15q11.2-q13.1 region and is characterized by developmental delay, autism spectrum disorder, and treatment resistant epilepsy. Extra copies of the E3 ubiquitin ligase $U B E 3 A$ and elevated levels of $U B E 3 A$ expression in neurons are thought to be the primary cause of Dup 15q phenotypes. However, animal models overexpressing $U B E 3 A$ in neurons have not successfully recapitulated all aspects of Dup15q syndrome, especially epilepsy. Here, we used Drosophila melanogaster (fruit flies) to investigate Dup 15q syndrome. In Chapter 2 we explored whether Dube $3 a$, the Drosophila homolog of $U B E 3 A$, is imprinted in the fly brain. In mammals, $U B E 3 A$ undergoes complex imprinting and is expressed only from the maternal allele in mature neurons. Prior to this work the imprinting status of Dube $3 a$ in flies was unclear. Here, we present evidence that Dube $3 a$ is not imprinted and is biallelically expressed in the fly. Next, in Chapter 3 we examined the interaction between Dube3a and HERC2. HERC2 is also an E3 ubiquitin ligase located in the 15q11.2-q13.1 critical region and is duplicated in all Dup15q individuals. HERC2 physically interacts with and stimulates the ubiquitin ligase activity of UBE3A in vitro. We found that Drosophila HERC2 appears to stimulate the ubiquitin ligase function of Dube3a, and Dube3a and HERC2 interact synergistically to impact phenotypes associated with Dup15q syndrome in vivo. Data presented in Chapter 3 suggests that genes other than $U B E 3 A$ are important in generating the Dup15q phenotypes and should not be ignored when modeling Dup15q syndrome. In Chapter 4 we investigated how non-neuronal cells, specifically glial cells, contribute to the seizure phenotype of Dup15q syndrome. We found that elevated levels of Dube3a or UBE3A in glia causes seizures, while overexpression in neurons does not. These data are consistent with mammalian models in which $U B E 3 A$ elevation in neurons does not generate seizures. Furthermore, overexpression of $D u b e 3 a$ in glia reduced protein levels of the $\mathrm{Na}^{+} / \mathrm{K}^{+}$ATPase, ATP $\alpha$. ATP $\alpha$ downregulation in glia is both necessary and sufficient to generate seizures. In Chapter 5 we further characterized our Dup15q epilepsy model and investigated cell type specific effects of Dube $3 a$ overexpression in glia compared to neurons using whole transcriptome and whole proteome analyses. We found that elevation of Dube $3 a$ in glia caused a cell non-autonomous downregulation of synaptic proteins in neurons while simultaneously causing a cell autonomous upregulation of glutathione S-transferases (GSTs) in glial cells. Finally, we showed that the upregulation of GSTs is common to multiple different Drosophila gliopathic seizure lines, not just our Dup15q epilepsy model. GSTs play a role in drug metabolism, and elevation of these enzymes may underlie the treatment resistant nature of some epilepsies including Dup $15 q$ syndrome. The results from these studies highlight the role that glial cell dysfunction may play in generating seizures in Dup15q syndrome and could ultimately provide novel avenues for epilepsy treatments.
\end{abstract}




\section{TABLE OF CONTENTS}

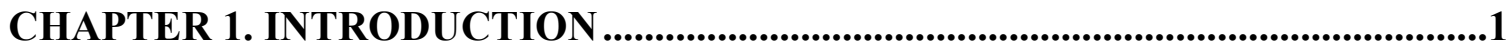

Duplication 15q Syndrome: Diagnosis and Subtypes.................................................

The Phenotypic Consequences of 15q11.2-q13 Duplications ......................................... 3

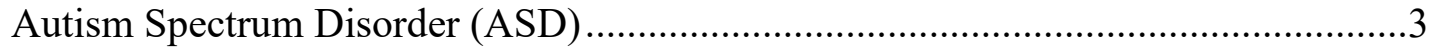

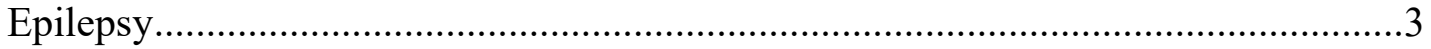

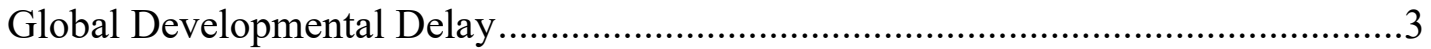

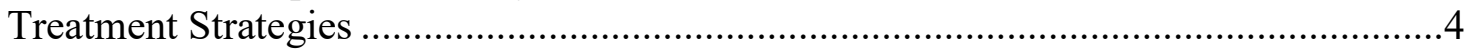

The Genetic and Molecular Basis of Dup15q Syndrome …….....................................

Genomic Origin of 15q Duplication ...................................................................

Genomic Imprinting Within the 15q11.2-q13.1 Locus.............................................

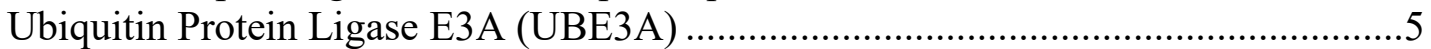

HECT and RLD Containing E3 Ubiquitin Ligase 2 (HERC2) ..................................

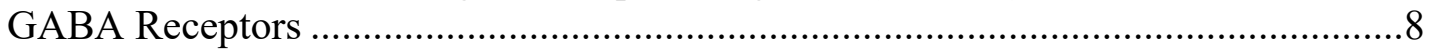

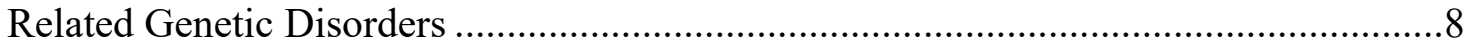

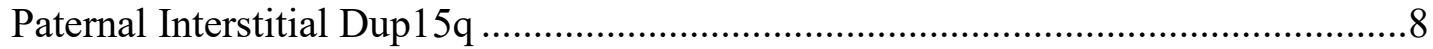

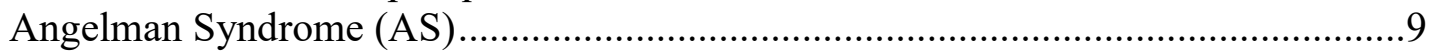

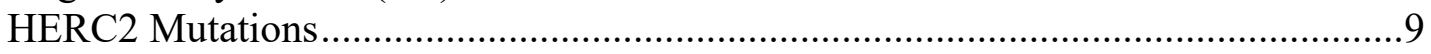

Dup15q Insights from Model Systems ………………............................................

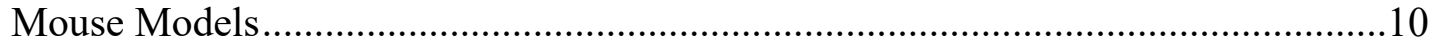

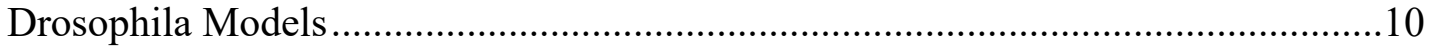

Hypothesis and Specific Aims .................................................................................

\section{CHAPTER 2. THE DROSOPHILA MELANOGASTER HOMOLOG OF UBE3A}

IS NOT IMPRINTED IN NEURONS...................................................................12

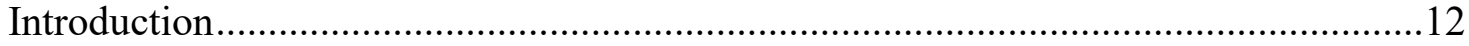

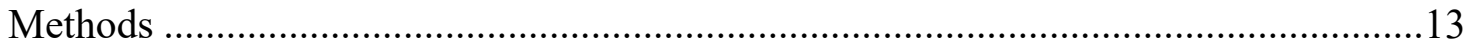

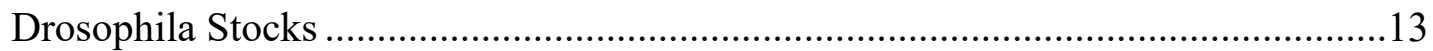

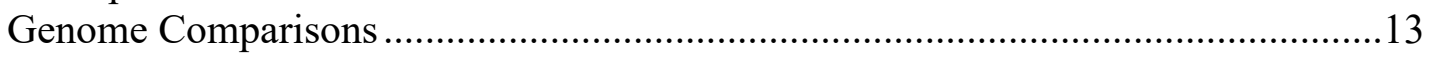

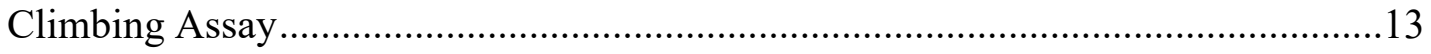

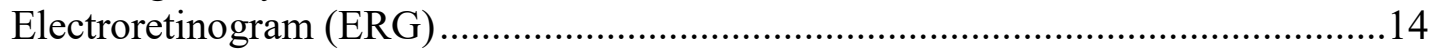

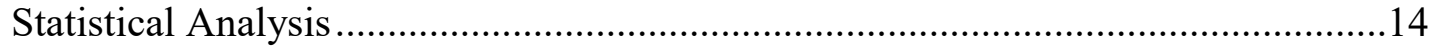

Allele-specific Dube3a Sequencing...................................................................14

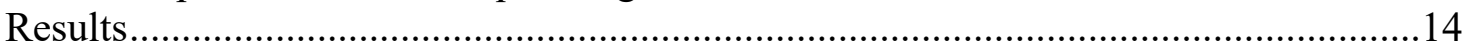

Genomic Regions Surrounding Fly and Human UBE3A are Not Syntenic ................14

Climbing Behavior is Not Dependent Upon Maternal Dube3a Expression ...............16

Synaptic Transmission Defects in Dube $3 a^{15 b}$ Mutants are Present Independent of

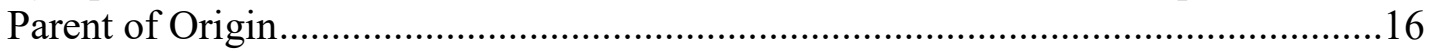

Allele-specific Expression of Dube $3 a$................................................................19

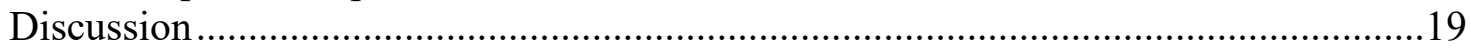




\section{CHAPTER 3. INVESTIGATING SYNERGISTIC INTERACTIONS BETWEEN DUBE3A AND HERC2}

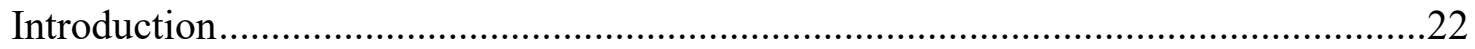

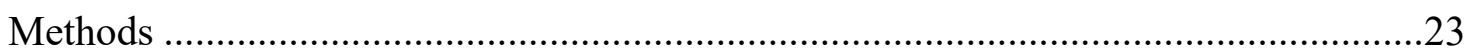

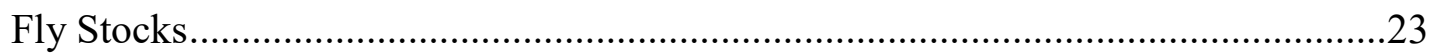

RLD2 Domain Comparison Between Human and Fly HERC2 .............................23

Quantitative Real-time Polymerase Chain Reaction (qRT-PCR) ..........................23

Quantitative Western Blot Analysis....................................................................23

Social Space Assay ....................................................................................24

Neuromuscular Junction Immunohistochemistry and Image Analysis.....................24

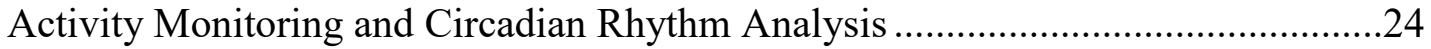

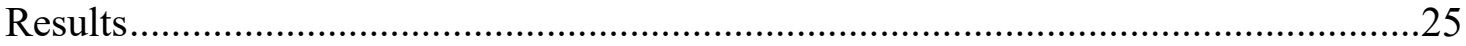

Human and Fly HERC2 Are Highly Conserved Within the RLD2 Domain .............25

Drosophila HERC2 Stimulates the Ubiquitin Ligase Activity of Dube3a................25

Simultaneous Overexpression of HERC2 and Dube $3 a$ Increases Social Spacing.....28

Overexpression of HERC2 and Dube3a Impairs Synaptic Morphology at the

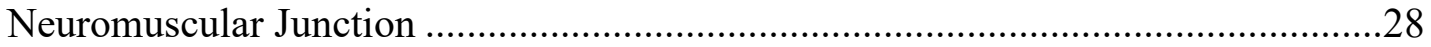

Co-overexpression of HERC2 and UBE3A in PDF Neurons Impairs Circadian

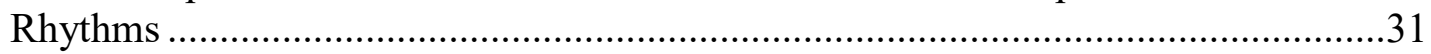

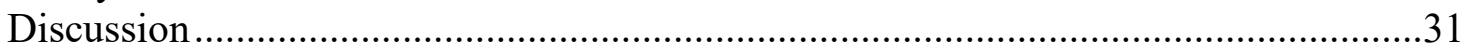

\section{CHAPTER 4. GLIAL OVEREXPRESSION OF DUBE3A CAUSES SEIZURES AND SYNAPTIC IMPAIRMENTS IN DROSOPHILA CONCOMITANT WITH DOWNREGULATION OF THE NA ${ }^{+} / \mathrm{K}^{+}$PUMP ATP-ALPHA .....................35}

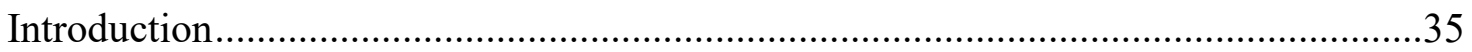

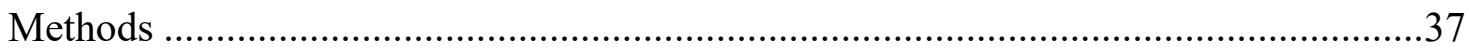

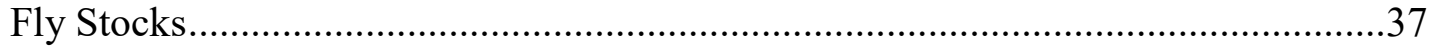

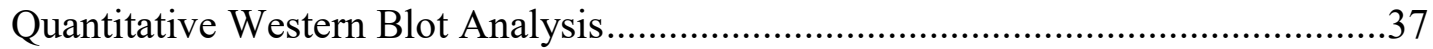

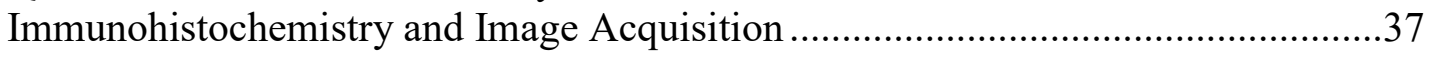

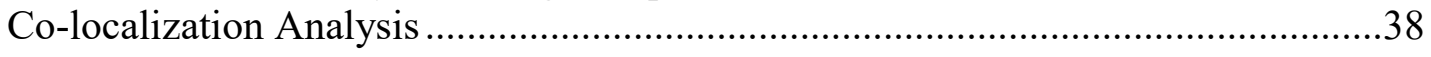

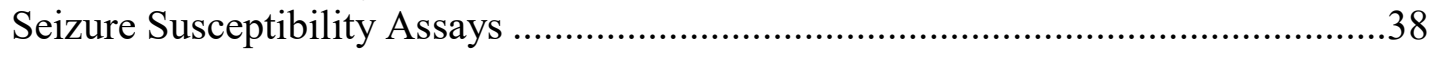

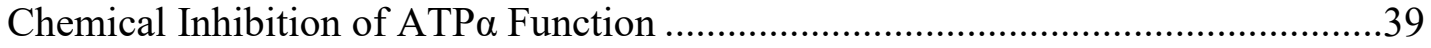

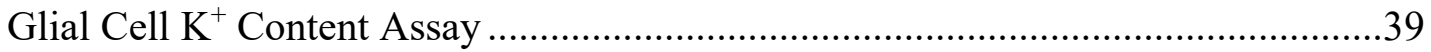

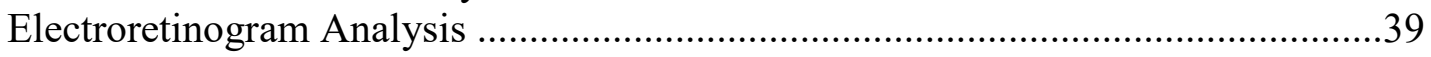

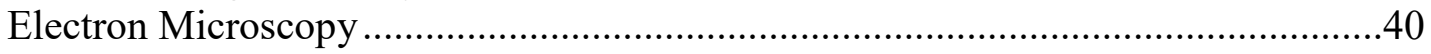

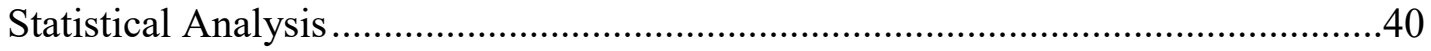

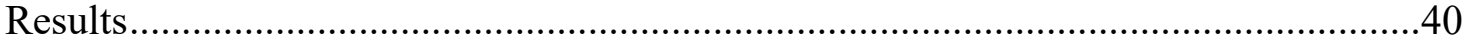

Glia Endogenously Express both Dube3a and ATP $\alpha$ Proteins .................................40

Overexpression of Dube $3 a$ in Glia Using repo-GAL4 Results in Seizure

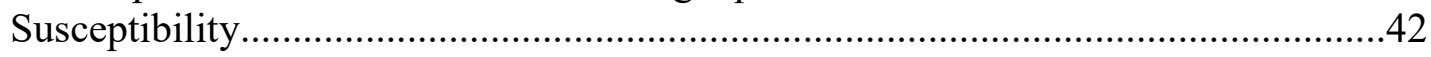

Genetic and Pharmacologic Manipulation of ATP $\alpha$ Modifies Seizure

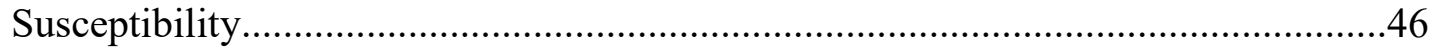

Glial Dube3a Overexpression Alters Neuronal Architecture .................................49

Overexpression of Dube $3 a$ in Glia, but Not Neurons, Impairs Photoreceptor

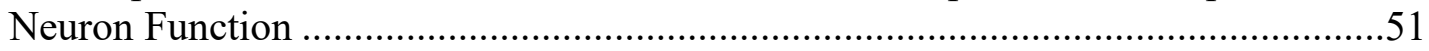


Glial-specific Overexpression of Dube3a Causes Reduced Intercellular $\mathrm{K}^{+}$in

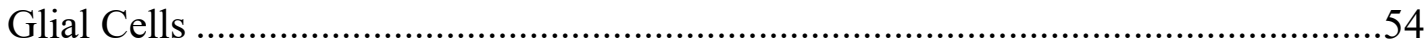

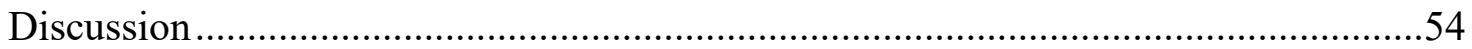

\section{CHAPTER 5. TRANSCRIPTOMIC AND PROTEOMIC PROFILING OF GLIAL VERSUS NEURONAL DUBE3A OVEREXPRESSION REVEALS COMMON MOLECULAR CHANGES IN GLIOPATHIC EPILEPSIES ................59}

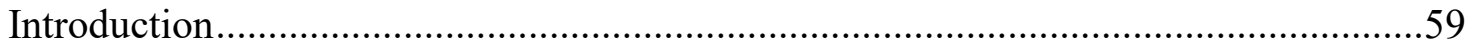

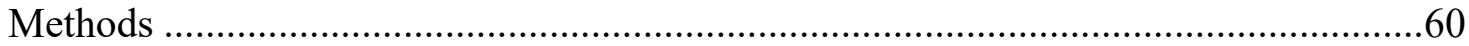

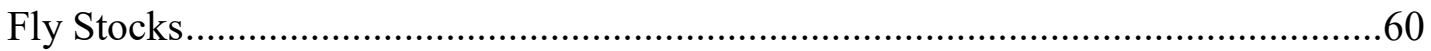

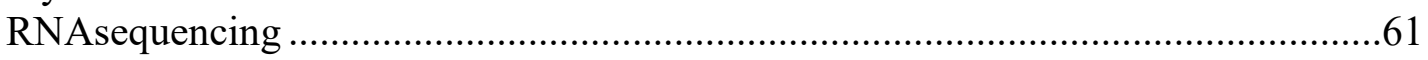

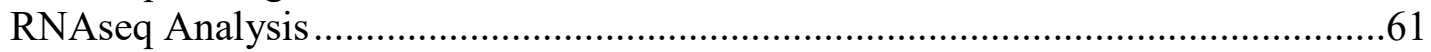

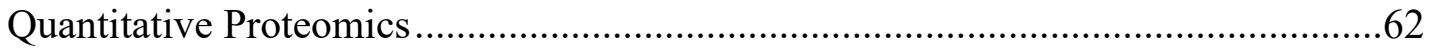

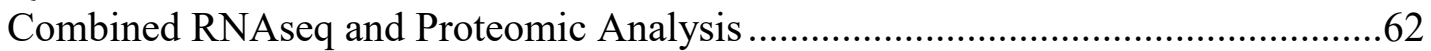

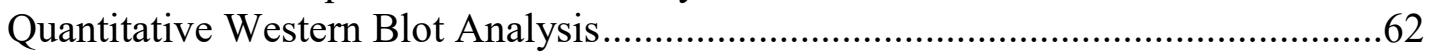

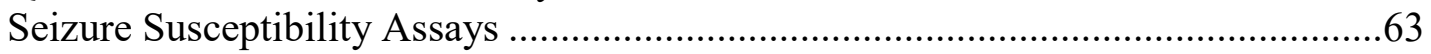

Microarray Analysis........................................................................................63

Quantitative Real-time Polymerase Chain Reaction (qRT-PCR) ……………….......64

Immunohistochemistry and Image Acquisition .......................................................64

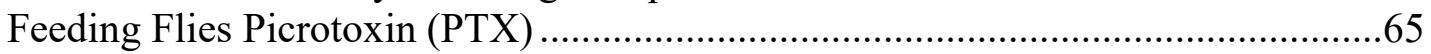

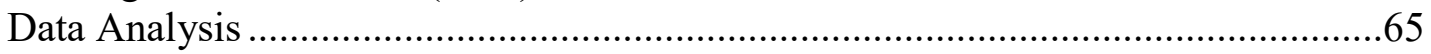

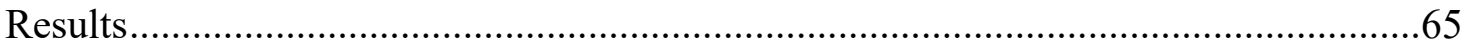

Transcriptomic Profiling Reveals an Upregulation of Glutathione S-transferases in repo > Dube 3 a Fly Heads ...............................................................................65

Proteomic Profiling Reveals Differential Expression of Neurotransmitter

Secretion Proteins in repo $>$ Dube $3 a$ Fly Heads .........................................................67

Comparison of the Transcriptome and the Proteome Reveals Downregulation of Synaptic Transmission Proteins in repo $>$ Dube $3 a$ Fly Heads

Glutathione S-transferase Upregulation is Common Among Gliopathic Seizure

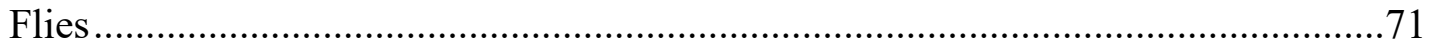

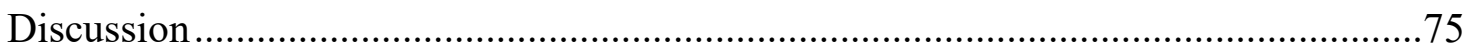

CHAPTER 6. DISCUSSION AND FUTURE DIRECTIONS .......................................77

Imprinting Status of UBE3A in Drosophila .............................................................77

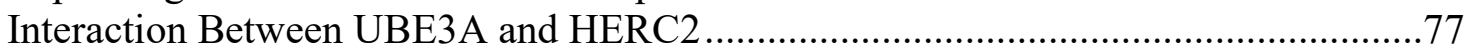

A Possible Role for Glia in Dup15q Epilepsy ............................................................78

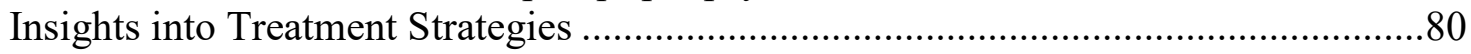

LIST OF REFERENCES .......................................................................................................82

VITA 


\section{LIST OF FIGURES}

Figure 1-1. Chromosome diagram of Dup15q subtypes............................................2

Figure 1-2. Mechanism of $U B E 3 A$ imprinting. .....................................................

Figure 2-1. The genomic region surrounding $U B E 3 A$ is not syntenic between flies

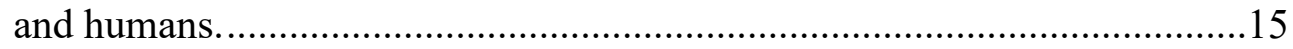

Figure 2-2. Only Dube $3 a^{15 b}$ homozygous LOF flies display climbing deficits..............17

Figure 2-3. Heterozygous Dube $3 a^{15 b}$ flies have reduced on/off transients in the ERG signal independent of parent of inheritance. ........................................... 18

Figure 2-4. Sanger sequencing chromatograms of different wild-type Dube3a coding SNPs does not indicate imprinting or preferential allele specific

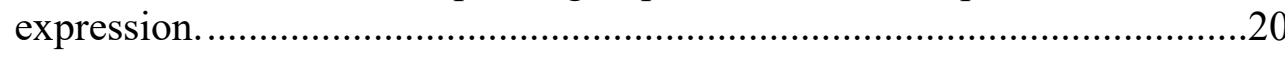

Figure 3-1. Human and Drosophila HERC2 proteins are homologous.........................26

Figure 3-2. Drosophila HERC2 stimulates the ubiquitin ligase activity of Dube3a. ......27

Figure 3-3. Simultaneous overexpression of $H E R C 2$ and Dube $3 a$ increases social spacing.

Figure 3-4. Overexpression of HERC2 and Dube3a impairs synaptic morphology at the neuromuscular junction.

Figure 3-5. Simultaneous HERC2 and Dube3a overexpression impairs circadian rhythms.

Figure 4-1. Glia endogenously express Dube $3 a$ and overexpression of Dube $3 a$ reduced ATP $\alpha$ protein levels in the brain.

Figure 4-2. Co-localization analysis results from Dube3a and ATP $\alpha$ signals in the fly optic lobe.

Figure 4-3. Elevated levels of Dube3a in glia result in seizure susceptibility in the bang-sensitivity seizure assay.

Figure 4-4. Seizures can be induced by heat or light in repo $>$ Dube3a animals.

Figure 4-5. Glial Dube3a overexpression in early development, but not adulthood, causes bang-sensitive seizures.

Figure 4-6. Genetic or pharmacologic manipulation of ATP $\alpha$ modulates seizure severity. 
Figure 4-7. Overexpression of Dube3a in glia alters mushroom body and optic lobe morphology.

Figure 4-8. Overexpression of Dube3a in glia alters ERG signals and reduces internal $\mathrm{K}^{+}$levels in glial cells.

Figure 4-9. Transmission electron microscopy of control (repo-GAL4) or glial Dube $3 a$ overexpression (repo $>$ Dube $3 a$ ) ommatidia of 5 day old flies.

Figure 4-10. Proposed mechanism of seizure in repo $>$ Dube $3 a$ animals. 56

Figure 5-1. Transcript profiling reveals an upregulation of GST genes in repo $>$ Dube3a flies.

Figure 5-2. Proteomic profiling reveals differential expression of neurotransmitter secretion proteins in repo $>$ Dube $3 a$ flies.

Figure 5-3. Cell non-autonomous downregulation of synaptic transmission proteins in repo $>$ Dube $3 a$ flies.

Figure 5-4. Glutathione S-transferase upregulation is common among multiple "gliopathic" seizure lines and occurs cell autonomously within glial cells

Figure 5-5. Glutathione S-transferase upregulation is not observed in flies fed PTX.....74

Figure 6-1. Unified working model of how elevated Dube3a in glia impacts the tripartite synapse. 


\section{LIST OF ABBREVIATIONS}

$\beta \mathrm{Gal}$

AED

ANOVA

APG-2

AS

ASD

AS-IC

$\mathrm{ATP} \alpha$

BDSC

BSA

cDNA

CNV

DAMS

DAVID

DD

DGRP

DMSO

DNA

DPSC

DSHB

Dube3a

Dup15

E6Ap

ERG

ES

FISH

GABA

GAL4

GAPDH

GFP

GO

GST

GstD1

HECT

HERC2

ID

iPSC

LCR

LOF

MB

mRNA

NMJ

PBS
Beta galactosidase

Antiepileptic drug

Analysis of variance

Asante potassium green 2

Angelman syndrome

Autism spectrum disorder

Angelman syndrome imprinting center

$\mathrm{Na}^{+} / \mathrm{K}^{+}$-ATPase $\alpha$-subunit

Bloomington drosophila stock center

Bang sensitivity assay

Complimentary deoxyribonucleic acid

Copy number variant

Drosophila activity monitoring system

Database for annotation, visualization, and integrated discovery

Developmental disability

Drosophila genetic reference panel

Dimethyl sulfoxide

Deoxyribonucleic acid

Dental pulp stem cells

Developmental studies hybridoma bank

Drosophila ubiquitin protein ligase E3A

Duplication $15 q$ syndrome

E6 associated protein

Electroretinogram

Enrichment score

Fluorescent in situ hybridization

Gaba-aminobutyric acid

Galactose-induced gene 4

Glyceraldehyde 3-phosphate dehydrogenase

Green fluorescent protein

Gene ontology

Glutathione S-transferase

Glutathione S-transferase D1

Homologous to the E6AP carboxyl terminus

HECT and RLD containing E3 ubiquitin ligase 2

Intellectual disability

Induced pluripotent stem cells

Low copy repeats

Loss of function

Mushroom body

Messenger ribonucleic acid

Neuromuscular junction

Phosphate buffered saline 


$\begin{array}{ll}\text { PDF } & \text { Pigment dispersing factor } \\ \text { PTX } & \text { Picrotoxin } \\ \text { PWACR } & \text { Prader-Willi/Angelman critical region } \\ \text { PWS-IC } & \text { Prader-Willi imprinting center } \\ \text { RCC1 } & \text { Regulator of chromosome condensation 1 } \\ \text { RIPA } & \text { Radio-immunoprecipitation assay } \\ \text { RLD } & \text { RCC1-like domain } \\ \text { RLD2 } & \text { RCC1-like domain 2 } \\ \text { RNA } & \text { Ribonucleic acid } \\ \text { SNP } & \text { Single nucleotide polymorphism } \\ \text { TBP } & \text { Tata-binding protein } \\ \text { UAS } & \text { Upstream activation sequence } \\ \text { UBE3A } & \text { Ubiquitin protein ligase E3A } \\ \text { UBE3A-AS } & \text { Ubiquitin protein ligase E3A antisense transcript }\end{array}$




\section{CHAPTER 1. INTRODUCTION}

\section{Duplication 15q Syndrome: Diagnosis and Subtypes}

The first cases of Duplication 15q syndrome (Dup15q) were reported in the early 1990 's. A young girl who presented clinically with delayed language development, ataxic gait, and global developmental delay was found to carry extra genetic material located on the q arm of her maternally inherited chromosome 15 (Clayton-Smith, Webb et al. 1993). At the same time a second group characterized a cohort of individuals carrying extra genetic material on chromosome $15 \mathrm{q}$, and based on the inverted nature of the chromosomal duplication the chromosomal abnormality was termed inv dup(15) (Robinson, Binkert et al. 1993), which is now broadly recognized as Dup15q syndrome.

Following these initial studies, the specific region of chromosome 15q that is duplicated has been identified. Dup15q is now known to be caused by the presence of at least one extra copy of the Prader Willi/Angelman critical region (PWACR) located within the 15q11.2-q13.1 region (Finucane, Lusk et al. 2016). Estimates of Dup15q prevalence rates vary. In individuals referred for clinical genetic testing by chromosomal microarrays due to hypotonia, motor delays, speech delays, autism spectrum disorder (ASD), or seizures, 15q11.2-q13.1 copy number gains are detected at a frequency of 1 in 508 (Moreno-De-Luca, Sanders et al. 2013). Multiple subtypes exist so further testing is required to identify the exact subtype of Dup15q. Fluorescent in situ hybridization (FISH) or cytogenetic analysis can determine whether a duplication resides within chromosome 15, termed interstitial Dup15q, or whether there is a supernumerary chromosome, termed isodicentric Dup15q. Parent of origin testing is useful in cases of interstitial Dup15q, however isodicentric Dup 15q appears to be only maternally derived and evidence for familial inheritance has not been reported. In cases of interstitial Dup15q, further genetic testing can reveal whether the duplication event was inherited maternally or paternally. Paternally inherited interstitial Dup $15 \mathrm{q}$ presents with a less severe phenotype and set of symptoms different from maternally derived duplications. Maternal interstitial Dup15q can be inherited familially if the mother carries a paternally derived interstitial duplication within her genome, so identifying parent of origin is important to determine if other relatives are at risk. See Figure 1-1 for a diagram of Dup15q subtypes.

Dup15q syndrome results in a number of disease phenotypes and both the presence and severity of these phenotypes differs among the Dup 15q subtypes, and even among individuals with the same genetic subtype. The phenotypes discussed in the following sections apply primarily to maternally derived duplications. Paternal interstitial Dup15q phenotypes will be discussed in a section on related genetic disorders. 

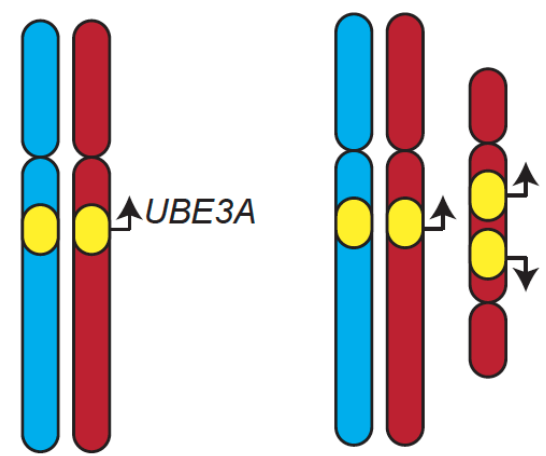

Typical

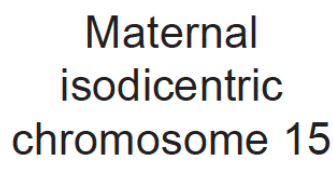

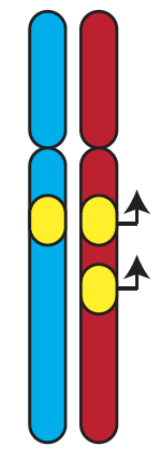

Maternal
interstitial
duplication

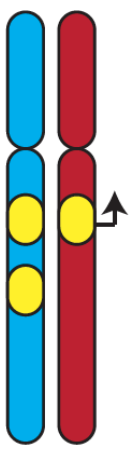

Paternal interstitial duplication

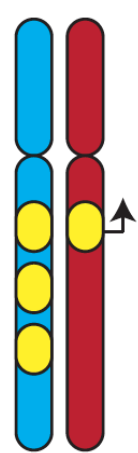

Paternal interstitial triplication

Figure 1-1. Chromosome diagram of Dup15q subtypes.

Typical individuals carry one 15q11.2-q13.1 region (yellow) on the paternal chromosome (blue) and one on the maternal chromosome (red). In cases of maternal isodicentric chromosome 15, a supernumerary chromosome is present that contains two extra copies of 15q11.2-q13.1. Maternal and paternal interstitial duplications can also occur creating one extra copy of the 15q11.2-q13.1 region within the chromosome. Additionally, paternal triplication cases can occur carrying two extra paternally derived copies of $15 \mathrm{q} 11.2-\mathrm{q} 13.1$. In this figure, $U B E 3 A$ is illustrated to be expressed only from the maternal chromosome, which occurs in neurons. In non-neuronal cell types, $U B E 3 A$ is expressed from both the maternal and paternal copies. 


\section{The Phenotypic Consequences of 15q11.2-q13 Duplications}

\section{Autism Spectrum Disorder (ASD)}

According to the DSM-V, ASD is defined by two core components. The first is impairments in social communication and social interaction across a broad range of contexts, which includes deficits in social-emotional reciprocity, nonverbal communication, and maintaining and understanding relationships. The second component is repetitive and restrictive behaviors consisting of repetitive movements, behavioral inflexibility, and fixated interests (American Psychiatric Association 2013). Greater than $50 \%$ of maternal interstitial duplications and greater than $80 \%$ of maternal isodicentric duplication individuals meet ASD diagnostic criteria (Hogart, Wu et al. 2010, Urraca, Cleary et al. 2013, Al Ageeli, Drunat et al. 2014). ASD in Dup15q shares many features with non-syndromic idiopathic ASD, the exception being one category of the ADOS-R test whereby responsive social smile and directed facial expressions are less impaired in the Dup15q population than the average in idiopathic ASD individuals (DiStefano, Gulsrud et al. 2016).

\section{Epilepsy}

Epilepsy is present at high rates in Dup15q syndrome and is one of the top concerns for the caregivers of Dup15q individuals. Seizure prevalence and severity is positively correlated with $15 \mathrm{q} 11.2-\mathrm{q} 13.1$ copy number with $25 \%$ of maternal interstitial, $64 \%$ of maternal isodicentric, and $100 \%$ of complex duplications (up to 6 extra copies) having seizures (Conant, Finucane et al. 2014). The majority of Dup15q cases present with multiple seizure types including tonic-clonic, atonic, myoclonic, and focal seizures, making epilepsy difficult to treat in this syndrome. Approximately half of Dup $15 q$ individuals with epilepsy develop treatment resistant refractory seizures (Conant, Finucane et al. 2014), severely impacting their quality of life. The mechanism of seizure in Dup15q remains poorly understood, and treatment options are limited.

\section{Global Developmental Delay}

Global developmental delay (DD) and impairment in fine motor skills is a feature that distinguishes Dup15q from other forms of nonsyndromic ASD (Hogart, Wu et al. 2010, DiStefano, Gulsrud et al. 2016). Dup15q is also characterized by walking impairments including increased stride width and a more varied gait pattern (Bhatt, Dickinson et al. 2018). Individuals with seizures present with lower IQ scores and greater impairments in motor function compared to Dup15q individuals without seizures (DiStefano, Gulsrud et al. 2016), suggesting that seizures impact cognitive and motor function beyond what is typically observed in the syndrome. However, the causal relationship remains unclear. 


\section{Treatment Strategies}

Due to the broad nature of the disease phenotypes a multidisciplinary approach is needed to effectively manage Dup $15 q$ syndrome. Physical therapy and augmented communication methods can be useful to manage hypotonia and speech impairments. Periodic seizure monitoring is recommended, as are broad spectrum antiepileptic drugs (AEDs) to manage seizures and avoiding known seizure triggers (reviewed in Finucane, Lusk et al. 2016). Some AEDs exacerbate seizures in the Dup15q population, making the epilepsy aspect of Dup15q particularly difficult to manage (Conant, Finucane et al. 2014). Unfortunately, treatment strategies are designed to address features of Dup15q, and mechanistic insights into the underlying causes of Dup15q phenotypes are needed in order to develop more effective, targeted treatments.

\section{The Genetic and Molecular Basis of Dup15q Syndrome}

\section{Genomic Origin of 15q Duplication}

There are several complex low copy repeats (LCRs) located on chromosome 15 that significantly increase inter- and intrachromosomal duplication events in this region (Bailey, Gu et al. 2002). The 15q11.2-q13.3 region contains 5 distinct complex LCRs, generating 5 distinct breakpoints for most duplication and deletion events. During meiosis I, unequal nonallelic homologous recombination can occur between these LCRs, generating the common deletions and duplications observed in this region (reviewed in $\mathrm{Gu}$, Zhang et al. 2008). Breakpoints 2-3 within the 15q11.2-q13.1 region contain the PWACR and this is the critical region that is duplicated in all Dup15q individuals. As predicted by the presence of LCRs, reciprocal deletions of the 15q11.2-q13.1 region also can occur. Depending on the parent of origin of the deletion, these events cause two distinct neurodevelopmental disorders, Angelman syndrome (AS) or Prader-Willi syndrome (PWS) (Hogart, Wu et al. 2010).

\section{Genomic Imprinting Within the 15q11.2-q13.1 Locus}

The Dup15q phenotype is variable depending on both copy number and the parent of origin of the duplication. Maternally derived duplications display a more severe phenotype than more rare paternal duplications (Cook, Lindgren et al. 1997, Schroer, Phelan et al. 1998, Urraca, Cleary et al. 2013), suggesting that critical genes in the region are subject to allele specific expression. Genomic imprinting refers to the process by which DNA is epigenetically marked, in this case through DNA methylation, resulting in differential gene expression dependent upon the parental origin of the DNA sequence. Two imprinting centers lie within the 15q11.2-q13.1 locus. The Angelman syndrome imprinting-center (AS-IC) is located upstream of the Prader-Willi syndrome imprintingcenter (PWS-IC) and regulates the methylation status of the PWS-IC. While the exact 
mechanism remains unclear, transcriptional activity at the AS-IC leads to DNA methylation at the PWS-IC within oocytes (Dittrich, Buiting et al. 1996, Kelsey and Feil 2013, Lewis, Brant et al. 2015), rendering the PWS-IC methylated and silenced on the maternally derived chromosome. The SNURF/SNRPN locus resides within the PWS-IC. The $S N U R F / S N R P N$ transcript is only expressed from the paternal allele due to the unmethylated status of the PWS-IC on the paternal chromosome. In mature mammalian neurons, the $S N U R F / S N R P N$ transcript progresses into the Ubiquitin protein ligase E3A $(U B E 3 A)$ gene and becomes the $U B E 3 A$-antisense transcript (UBE3A-AS). The $U B E 3 A$ $A S$ is on the opposite DNA strand of $U B E 3 A$. Expression of $U B E 3 A-A S$ sterically interferes with expression of $U B E 3 A$ on the paternal chromosome, rendering paternal $U B E 3 A$ imprinted and silenced in mature mammalian neurons (reviewed in LaSalle, Reiter et al. 2015). In non-neuronal cell types, the $S N U R F / S N R P N$ transcript does not progress into the $U B E 3 A$ gene and both the maternal and paternal copy of $U B E 3 A$ are expressed. See Figure 1-2 for a diagram of $U B E 3 A$ imprinting.

\section{Ubiquitin Protein Ligase E3A (UBE3A)}

The maternally imprinted $U B E 3 A$ gene, located within the $15 \mathrm{q} 11.2-\mathrm{q} 13.1$ critical region, is thought to play a primary role in the etiology of Dup15q syndrome phenotypes. $U B E 3 A$, also known as E6 associated protein (E6AP), was originally identified as the ubiquitin ligase that interacts directly with the human papillomavirus (HPV) E6 oncoprotein to cause the degradation of p53 during viral infection (Huibregtse, Scheffner et al. 1993). The primary role of UBE3A as an E3 ubiquitin ligase is to build K48 linked polyubiquitin chains on substrate proteins (Kim and Huibregtse 2009), signaling these proteins for degradation by the 26S proteasome (Zheng and Shabek 2017). HPV-16 causes cervical cancer by introducing the E6 viral protein into a cell, resulting in ubiquitination and degradation of the tumor suppressor p53 by UBE3A and leading to unchecked cell growth and carcinogenesis (Scheffner, Huibregtse et al. 1993). It is important to note that when E6 viral oncoprotein is not present, UBE3A does not target p53 for ubiquitination.

$U B E 3 A$ is the founding member of the homologous to the E6AP carboxyl terminus (HECT) domain E3 ligase family of ubiquitin ligase proteins. The C-terminus of UBE3A contains the active site responsible for substrate protein ubiquitination. All HECT domain protein family members contain a region of 350 amino acids homologous to the $\mathrm{C}$-terminus of UBE3A. The $\mathrm{C}$-terminus of $\mathrm{UBE} 3 \mathrm{~A}$ is responsible for interacting with cognate $\mathrm{E} 2$ ligases that carry activated ubiquitin, while the $\mathrm{N}$-terminus is involved in substrate protein identification. Human $U B E 3 A$ exists in at least three different isoforms and is predicted to be expressed in both the nucleus and cytoplasm (reviewed in LaSalle, Reiter et al. 2015). Immunohistochemistry on human brain tissue indicates that UBE3A is present in both the nucleus and cytoplasm of neurons, including synaptic terminals and dendrites, and is also detected in oligodendrocytes and astrocytes in human brain tissue (Burette, Judson et al. 2018). Studies from postmortem Dup15q brain tissue (Scoles, Urraca et al. 2011) and Dup15q patient derived dental pulp stem cell neuron cultures 

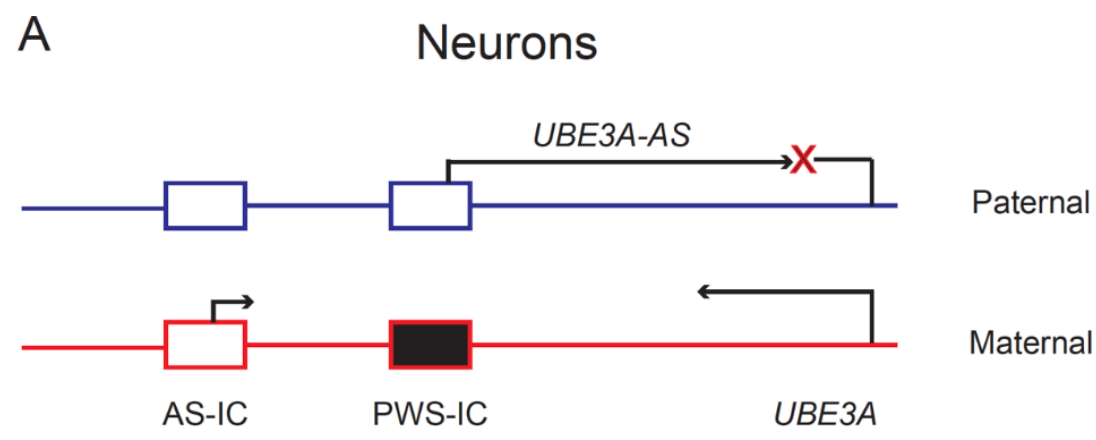

B Non-neuronal cells

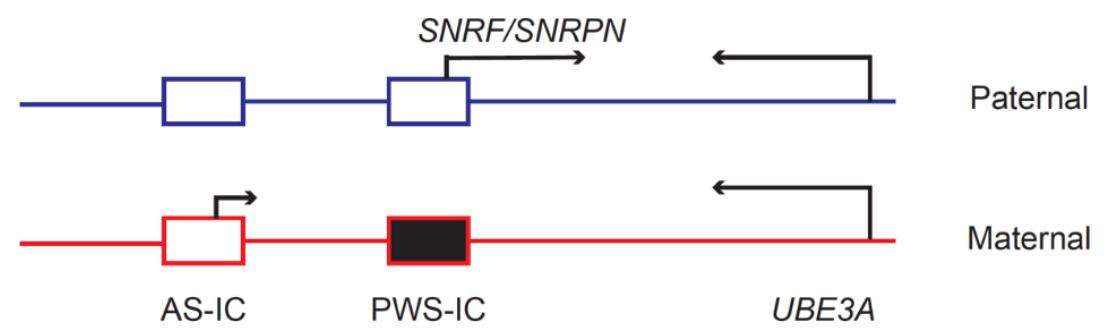

Figure 1-2. Mechanism of $U B E 3 A$ imprinting.

A) In neurons, $U B E 3 A$ is paternally silenced and only expressed from the maternal allele of chromosome 15. Transcriptional activity at the maternal Angelman syndrome-

imprinting center (AS-IC) leads to methylation of the Prader-Willi syndrome-imprinting center (PWS-IC) which shuts down expression of the $U B E 3 A-A S$ on the maternal copy. However, on the paternal chromosome, the PWS-IC is unmethylated and the $U B E 3 A-A S$ sterically interferes with expression of $U B E 3 A$, leading to $U B E 3 A$ silencing. B) In nonneuronal cells, the $S N R F / S N R P N$ transcript expressed from the PWS-IC on the paternal chromosome does not progress into the $U B E 3 A$ gene and, therefore, no $U B E 3 A-A S$ is present. As such, $U B E 3 A$ is biallelically expressed in many non-neuronal cell types. 
(Urraca, Hope et al. 2018) indicate increased levels of $U B E 3 A$ transcript and protein in the brains of Dup15q individuals, suggesting increased UBE3A-dependent protein degradation as the primary cause of Dup15q phenotypes.

The primary role of $\mathrm{UBE} 3 \mathrm{~A}$ is to function an E3 ubiquitin ligase that targets particular substrate proteins in the cell for degradation by the ubiquitin proteasome. In the presence of an E2 ligase and a substrate protein, the catalytic cysteine residue in the UBE3A HECT domain forms a thioester bond with ubiquitin before transferring the ubiquitin to lysine residues on the substrate protein. UBE3A preferentially forms K48 linked polyubiquitin chains on substrate proteins in a stepwise manner, targeting these proteins for degradation by the 26S proteasome (reviewed in Scheffner and Kumar 2014). Consistent with UBE3A being present in both the cytosol and the nucleus, ubiquitin substrates of UBE3A have been reported from both cellular compartments including RING1B/RNF2 from the nucleus and RPN10/PSMD4 and Ephexin V from the cytosol, among other proteins (LaSalle, Reiter et al. 2015). Additionally, UBE3A has been reported to ubiquitinate substrate proteins without targeting them for degradation, possibly altering their subcellular localization or function (Reiter, Seagroves et al. 2006, Ramirez, Lectez et al. 2018). In another role independent of its ubiquitin ligase function, UBE3A can also act as a transcriptional co-activator. In the nucleus, UBE3A induces transcription of nuclear hormone receptors (Nawaz, Lonard et al. 1999) and GTP cyclohydrolase 1 (Ferdousy, Bodeen et al. 2011), an enzyme regulating monoamine synthesis. Initial disease focused investigations of UBE3A proposed the concept that a single UBE3A substrate may be identified to explain the pathology of both AS and Dup15q. Despite a growing list of known UBE3A interacting proteins, no one substrate can fully explain both disorders and the complexity of UBE3A regulation remains enigmatic.

\section{HECT and RLD Containing E3 Ubiquitin Ligase 2 (HERC2)}

A second ubiquitin ligase, HERC2, is also located within PWACR and is duplicated in all individuals with Dup15q. The rjs (runty jerky sterile) mouse, named for its jerky gait, reduced growth, sterility, and maternal behavioral defects carries a loss of function mutation in Herc2 (Lehman, Nakatsu et al. 1998), demonstrating a role for HERC2 in normal neuronal development. HERC2 is a large protein consisting of 4834 amino acids and multiple protein domains and is expressed throughout the brain, including the cortex, hippocampus, and cerebellum (Cubillos-Rojas, Schneider et al. 2016). Similar to UBE3A, the C-terminus of HERC2 contains a catalytic HECT domain responsible for transferring ubiquitin moieties to substrate proteins. HERC2 also functions in the nucleus to facilitate DNA repair in response to DNA double strand breaks (Bekker-Jensen, Rendtlew Danielsen et al. 2010), ubiquitinates and causes the degradation of the deubiquitinating enzyme USP33 (Chan, den Besten et al. 2014), and regulates the integrity of the centrosome (Al-Hakim, Bashkurov et al. 2012). In addition to the HECT domain, HERC2 contains 3 RCC1-like domains (RLD), consisting of regulator of chromosome condensation-1 (RCC1) repeats. HERC2 is known to physically interact with UBE3A at the protein level through the RLD2 domain and stimulates 
UBE3A ubiquitin ligase activity independent of the HECT domain of HERC2 (Kuhnle, Kogel et al. 2011). The interaction between UBE3A and HERC2 has largely been studied in vitro using biochemical methods, so how these two proteins interact to generate Dup15q phenotypes is still unknown.

\section{GABA Receptors}

A cluster of GABA receptors, including GABRA5, GABRB3, and GABRG3 are present within breakpoints 2-3 in the 15q11.2-q13.1 region and are duplicated in all cases of Dup15q. Spontaneous beta oscillations detected in electroencephalograms (EEGs) of Dup15q individuals, reminiscent of an EEG pattern generated by GABAergic promoting benzodiazepines and barbiturates, suggests altered GABAergic signaling in Dup15q syndrome (Urraca, Cleary et al. 2013, Frohlich, Senturk et al. 2016). Researchers often implicate the duplication of these GABA receptors in Dup15q epilepsy, yet robust evidence is lacking. Missense variants that impair GABRA5 and GABRB3 receptor activity are associated with early onset epilepsy (Butler, Moody et al. 2018), however missense variants are not the same as extra gene copies. Furthermore, 15q11.2-q13.1 copy number does not correlate with GABA receptor transcript levels (Scoles, Urraca et al. 2011, Urraca, Hope et al. 2018), making it unclear how extra copies of these receptors tie into Dup15q syndrome phenotypes.

\section{Related Genetic Disorders}

\section{Paternal Interstitial Dup15q}

Individuals carrying paternally derived interstitial duplications present clinically with a more variable phenotype compared to maternal duplications. In addition, paternally derived duplications display incomplete penetrance, as some completely unaffected individuals have been identified in pedigrees where this duplication was inherited and not de novo in origin (Cook, Lindgren et al. 1997). Parasomnias are a consistent phenotype observed in paternal Dup15q individuals, however ASD and IQ levels can be more variable (Urraca, Cleary et al. 2013). In a cohort of 522 ASD patients, one individual was identified carrying a paternal interstitial duplication of 15q11.2-q13.1 while no maternally derived interstitial cases were found (Depienne, Moreno-De-Luca et al. 2009), implying that paternal duplications may also cause ASD, although with variable expressivity and penetrance. Cases of paternally derived triplications have also been reported. These individuals display phenotypes more consistent with maternal duplications, including developmental delay, hypotonia, language deficits, and seizures (Hogart, Wu et al. 2010), suggesting that genes within the duplication, other than the neuronally maternally imprinted $U B E 3 A$ gene, can contribute to the disease phenotype when elevated at higher levels. Additionally, glial cells biallelically express $U B E 3 A$ and glial $U B E 3 A$ overexpression may contribute to the seizure phenotype of paternal interstitial triplication cases where glia, but not neurons, express two extra copies of 
$U B E 3 A$. Seizure prevalence increases with 15q11.2-q13.1 copy number regardless of the parent of origin, implying that elevated levels of UBE3A in glia could be associated with the seizure phenotype. However, the impact of glial $U B E 3 A$ overexpression has gone largely unexplored.

\section{Angelman Syndrome (AS)}

Maternal deletions of 15q11.2-q13.1, the reciprocal non-homologous allelic recombination event to the duplications resulting in Dup15q syndrome, are the most common cause of AS. However, maternally inherited loss of function mutations in $U B E 3 A$, paternal uniparental disomy of chromosome 15, or imprinting defects rendering the PWSIC unmethylated can also cause AS (LaSalle, Reiter et al. 2015). Regardless of the molecular genetic event, the underlying molecular cause of AS is the loss of UBE3A expression in neurons. AS is clinically characterized by microcephaly, hypotonia and motor delay, sleep disturbances, and the specific behavioral phenotype of excessive laughter (Buiting, Williams et al. 2016). Similar to Dup15q, AS individuals have high rates of epilepsy, although these seizures are distinct by EEG from the types of seizure observed in Dup15q syndrome and can be managed well with certain anti-epileptics (Pelc, Boyd et al. 2008, Shaaya, Grocott et al. 2016). Seizures are less severe in UBE3A loss-of-function, uniparental disomy, or methylation defect cases compared to deletion cases (Minassian, DeLorey et al. 1998, Frohlich, Miller et al. 2019) suggesting that genes other than $U B E 3 A$ within the 15q11.2-q13.1 region impact the epilepsy phenotype.

\section{HERC2 Mutations}

Loss of function HERC2 mutations can also generate AS-like phenotypes. Three separate cohorts with developmental delay, ASD, and Angelman-like features have been identified and individuals carried homozygous mutations in HERC2 that either reduced HERC2 protein stability or deleted large sections of HERC2 including the RLD2 domain that stimulates UBE3A ubiquitin ligase activity (Puffenberger, Jinks et al. 2012, Harlalka, Baple et al. 2013, Morice-Picard, Benard et al. 2016)). The overlapping phenotypes between $H E R C 2$ loss of function mutations and AS provides further evidence for the interaction between UBE3A and HERC2 in vivo and implies that HERC2 protein may be necessary for the normal function of UBE3A. The physical location of the HERC2 gene within the PWACR that is duplicated in Dup15q and deleted in most cases of AS further implies that HERC2 may be a modifier of the phenotypes observed in both of these disorders. 


\section{Dup15q Insights from Model Systems}

\section{Mouse Models}

The animal model with the best face validity of Dup15q is a mouse carrying an interstitial duplication of $6.3 \mathrm{Mb}$ of mouse chromosome 7 , a region syntenic to the 15q11.2-q13.1 region in humans. Mice carrying paternally inherited duplications displayed ASD-like behaviors including reduced social interactions, behavioral inflexibility, and lower levels of serotonin in the cortex (Nakatani, Tamada et al. 2009, Nakai, Nagano et al. 2017). However, these phenotypes were not observed in maternally inherited duplications which is inconsistent with the parent of origin effect in humans. This mouse model does however suggest that genes other than Ube3a or elevation of Ube3a in non-neuronal cell types impacts the phenotypes associated with Dup15q.

Mouse models overexpressing only Ube $3 a$ have also been generated. The first model carried extra copies of C-terminal FLAG tagged Ube3a and recapitulated ASDlike behaviors (Smith, Zhou et al. 2011), but the addition of a FLAG tag to the Cterminus of Ube3a rendered the protein catalytically inactive, and this data has not been reproducible by other groups. A second mouse was generated by the same group overexpressing untagged Ube $3 a$ and again they observed sociability deficits. These mice had decreased sociability when seizures were chemically induced, however these mice did not show spontaneous seizures nor reduced seizure threshold (Krishnan, Stoppel et al. 2017). A second group generated mice overexpressing Ube $3 a$ under control of the Camk2a promoter and mice displayed anxiety-like behavior, learning deficits, and reduced seizure threshold to pentylenetetrazol. However, ASD-like behaviors and spontaneous seizures were not observed (Copping, Christian et al. 2017). Taken as a whole, data from mouse models suggests that elevated levels of $U b e 3 a$ in neurons may underlie the ASD-like and cognitive impairment aspects of Dup15q syndrome, however neuronal Ube3a elevation does not appear to be responsible for epilepsy in Dup15q.

\section{Drosophila Models}

Despite the divergence of Homo sapiens and Drosophila melanogaster over 700 million years ago, $46 \%$ of fly genes have an ortholog to at least one gene in the human genome (Shih, Hodge et al. 2015) and approximately $75 \%$ of human disease genes have a clear fly ortholog (Reiter, Potocki et al. 2001). Based on the high degree of conservation of disease genes, quick generation time, and the number of tools available (Ugur, Chen et al. 2016), fruit flies are an attractive system to investigate human disease. Drosophila have a single ortholog of $U B E 3 A$, termed Dube $3 a$, which is $40 \%$ conserved across the whole protein and greater than $70 \%$ identical in the HECT domain compared to human UBE3A (Reiter, Seagroves et al. 2006). Based on the conservation between Dube3a and UBE3A, Drosophila is a suitable model system and multiple fly models have been generated to investigate UBE3A function. 
By taking advantage of the GAL4/UAS system (Duffy 2002), a handful of direct ubiquitin ligase substrates of Dube3a have been identified using flies, including the RhoGEF Pbl (Reiter, Seagroves et al. 2006), the $\mathrm{Na}^{+} / \mathrm{K}^{+}$ATPase ATP $\alpha$ (Jensen, Farook et al. 2013), and the proteasomal proteins Rpn10 (Lee, Ramirez et al. 2014) and Rngo (Ramirez, Lectez et al. 2018). Elevated levels of Dube3a in motor neurons results in increased excitability and reduced capacity to maintain high firing frequencies (Valdez, Scroggs et al. 2015), and neuronal overexpression throughout the fly brain results in learning defects in an aversive phototaxis suppression assay (Chakraborty, Paul et al. 2015). Insights have also been made from Dube3a null mutant flies. Loss of Dube $3 a$ results in impaired locomotion and circadian rhythm defects (Wu, Bolduc et al. 2008) and decreased arborization in the dendrites of peripheral neurons (Lu, Wang et al. 2009), recapitulating some aspects of Angelman syndrome. Additionally, a lesser known transcriptional co-activation function of Dube3a has been investigated in Drosophila in which even a ubiquitin ligase defective form of Dube3a elevates levels of tetrahydrobiopterin, the rate limiting cofactor in the synthesis of monoamines (Ferdousy, Bodeen et al. 2011). Despite a number of models that focus on neuronal functions for $U B E 3 A$ and the substrates that have been identified, no model has yet successfully recapitulated the seizure phenotype observed in Dup15q syndrome.

\section{Hypothesis and Specific Aims}

$U B E 3 A$ plays a key role in Dup15q syndrome, yet the mechanism underlying Dup 15q phenotypes, particularly seizures, remains poorly understood. First, we wanted to determine the imprinting status of Dube3a in Drosophila melanogaster. Next, we hypothesized that genes located within the 15q11.2-q13.1 duplication region other than $U B E 3 A$ act alone or synergistically with $U B E 3 A$ to impact Dup15q phenotypes. Based on the known interaction between UBE3A and HERC2, we decided to investigate the interaction between these two proteins. Finally, we hypothesized that cells other than neurons underlie aspects of Dup15q syndrome, particularly the seizure phenotype. To test these hypotheses, we proposed the following specific aims:

1. Determine if Dube3a is imprinted in Drosophila melanogaster. Data from these experiments are presented in Chapter 2.

2. Investigate if Dube $3 a$ and HERC2 interact synergistically to generate Dup $15 \mathrm{q}$ phenotypes. Data from these experiments are presented in Chapter 3.

3. Identify if non-neuronal cells, specifically glia, are involved in the seizures observed in Dup15q syndrome. Data from these experiments are presented in Chapters 4 and 5. 


\section{CHAPTER 2. THE DROSOPHILA MELANOGASTER HOMOLOG OF UBE3A IS NOT IMPRINTED IN NEURONS ${ }^{1}$}

\section{Introduction}

Angelman syndrome (AS) results from neuronal loss of maternally expressed ubiquitin protein ligase E3A (UBE3A) in neurons (Kishino, Lalande et al. 1997). Maternal deletions of chromosome 15q11.2-q13.1 encompassing $U B E 3 A$ are the most common cause of AS. However, maternally-inherited loss-of-function (LOF) point mutations in $U B E 3 A$, imprinting defects, or paternal uniparental disomy also cause AS (LaSalle, Reiter et al. 2015). The first evidence for imprinted expression of $U B E 3 A$ in neurons arose from the observation that maternally derived deletions of 15q11.2-q13.1 cause AS (Knoll, Nicholls et al. 1989) and that UBE3A displays maternal allele-specific expression in human brain samples (Rougeulle, Glatt et al. 1997). In mice, Ube3a is expressed from the maternal allele in hippocampal, cerebellar, and olfactory bulb mitral neurons (Albrecht, Sutcliffe et al. 1997). Ube3a is biallelically expressed in neurons born from stem cells in the adult mammalian brain and immature neurons early in development (Dindot, Antalffy et al. 2008). Ube3a expression shifts from biallelic to maternal allele specific as neurons mature (Judson, Sosa-Pagan et al. 2014). UBE3A is biallellically expressed in human induced pluripotent stem cells and neuronal differentiation results in paternal silencing of $U B E 3 A$ (Chamberlain, Chen et al. 2010). In contrast to neurons, glial cells in the mammalian brain biallelically express $U B E 3 A$ (Yamasaki, Joh et al. 2003, Dindot, Antalffy et al. 2008, Judson, Sosa-Pagan et al. 2014).

Imprinted expression of $U B E 3 A$ in neurons is a complex mechanism mediated by the expression of a $U B E 3 A$-antisense transcript $(U B E 3 A-A S)$ that interferes with expression of the sense $U B E 3 A$ full-length transcript. The Prader-Willi syndrome imprinting center (PWS-IC) is maternally methylated and silenced due to an upstream Angelman syndrome imprinting center (AS-IC) that epigenetically alters the maternal PWS-IC in the female germline via a transcriptional mechanism, leaving maternal PWSIC nonfunctional post-fertilization (Kelsey and Feil 2013). Downstream from the PWSIC is the $S N U R F-S N R P N$ locus, and the $S N R P N$ transcript progresses through a cluster of snoRNAs and spliced host genes, terminating as $U B E 3 A-A S$. The expression of $U B E 3 A-$ $A S$ on the opposite strand of $U B E 3 A$ interferes with $U B E 3 A$ expression on the paternal allele and is the mechanism for paternal $U B E 3 A$ silencing (LaSalle, Reiter et al. 2015).

Although it is well-established that $U B E 3 A$ is imprinted in the mature neurons of human and mouse brain, it is unclear if $U B E 3 A$ is imprinted in the brains of nonmammalian species including the model invertebrate organism Drosophila melanogaster. Flies have a single $U B E 3 A$ homolog (Reiter, Seagroves et al. 2006), Dube $3 a$, and mutations in this gene give rise to various phenotypes independent of the parental origin

\footnotetext{
${ }^{1}$ Adapted with open access permission from final submission. Hope, K.A., LeDoux, M.S., and Reiter, L.T. (2016). The Drosophila melanogaster homolog of UBE3A is not imprinted in neurons. Epigenetics. 11(9), 637-642. DOI: 10.1080/15592294.2016.1214783.
} 
of the mutation (Wu, Bolduc et al. 2008). Recently, it was reported that Dube3a is preferentially expressed from the maternal allele in flies (Chakraborty, Paul et al. 2015). However, there is a lack of synteny between fly and human genomes at the $U B E 3 A / D u b e 3 a$ loci and there is no prior evidence for allele specific expression in flies. Given that flies do not have a documented imprinting center, differentially methylated region or $U B E 3 A-A S$, we set out to definitively determine if Dube $3 a$ is, in fact, preferentially expressed from the maternal allele in fly brain. Using behavioral analysis, electroretinograms (ERGs) and allele-specific coding single nucleotide polymorphism (SNP) expression studies, we found no evidence for Dube3a imprinting in flies. Both maternal and paternal Dube $3 a$ alleles are co-expressed in fly brain.

\section{Methods}

\section{Drosophila Stocks}

All flies were maintained at $25^{\circ} \mathrm{C}$ on a 12-hour light/dark cycle and raised on standard corn meal media (Bloomington Stock Center). Dube $3 a^{15 b}$ is a null loss-offunction (LOF) allele that produces a truncated transcript that does not make functional Dube3a protein (Reiter, Seagroves et al. 2006). $w^{1118}$ flies were used as controls. The following Drosophila Genetic Reference Panel (DGRP) lines (Mackay, Richards et al. 2012) were also obtained from the Bloomington Drosophila Stock Center: RAL-21, RAL-239, RAL-313, and RAL-517 (stock numbers 28112, 28161, 25180, and 25197, respectively).

\section{Genome Comparisons}

Genomic regions surrounding Dube $3 a$ in Drosophila melanogaster were compared to human UBE3A using the UCSC genome browser (http://genome.ucsc.edu/) (Kent, Sugnet et al. 2002). We used fly BDGP Release 6 + ISO1 MT/dm6 assembly and human GRCh38/hg38 assembly for comparisons.

\section{Climbing Assay}

Climbing assays were performed as previously described (Hatfield, Harvey et al. 2015). In brief, 3 flies of each genotype at $5 \mathrm{~d}$ post-eclosion were transferred to empty vials and allowed $1 \mathrm{~min}$ to acclimate. Flies were tapped to the bottom of the vial and the time was measured for the first fly to climb past a $4 \mathrm{~cm}$ vertical mark. Three trials were averaged per group of flies with 1 min intervals. 


\section{Electroretinogram (ERG)}

ERGs were performed as previously described (Vilinsky and Johnson 2012), with modifications. Flies were immobilized on ice for $2 \mathrm{~min}$, transferred to a glass slide, and held in place with dental wax. Tungsten wire electrodes were gently inserted into the right eye (recording electrode) and abdomen (ground electrode). Data was recorded using a Model 1800 Microelectrode AC Amplifier (A-M Systems), digitized with a Micro3 1401 digitizer (Cambridge Electronic Design), and analyzed with Spike2 software (Cambridge Electronic Design). Fly eyes were stimulated using 1-s light pulses from a 5 mm white LED (Radioshack).

\section{Statistical Analysis}

Significance testing was performed by 1-way ANOVA analysis. All statistics were performed using Prism version 6.0 (GraphPad).

\section{Allele-specific Dube3a Sequencing}

Using the DGRP and the UCSC Genome browser of DGRP2 data (dgrp2.gnets.ncsu.edu), we selected 3 lines with single nucleotide polymorphisms (SNPs) in coding exons of Dube3a. RAL-21 was used as a reference line, and lines RAL-313, RAL-239, and RAL-517 harbored G78T, G117T, and C171A SNPs, respectively. We performed reciprocal crosses and extracted RNA from heads for cDNA synthesis. Totals of 20-30 fly heads from each cross were removed and homogenized in TRI Reagent Solution (Ambion). Total RNA was extracted using Directzol RNA MiniPrep Plus (Zymo Research Corp) according to the manufacturer's instructions, which include a DNAse step to remove genomic DNA. RNA was converted to cDNA using Transcriptor First Strand cDNA Synthesis Kit (Roche). A 950 bp fragment of Dube3a encompassing our chosen SNPs was PCR amplified with Phusion polymerase (Thermofisher Scientific) using the following forward and reverse primers, respectively: 5'ATGAACGGTGGCGGGG-3', and 5'-CGCTGCTTTGGGATGAACAC-3'. PCR products were sent for Sanger sequencing (GENEWIZ) using the following sequencing primer: 5'-AATGAAGCTCTTGCCCAGTC-3'. Sequence data was analyzed using CodonCode Aligner (CodonCode Corporation).

\section{Results}

\section{Genomic Regions Surrounding Fly and Human $U B E 3 A$ are Not Syntenic}

The UCSC genome browser was used to examine genomic features surrounding fly $D u b e 3 a$ and human $U B E 3 A$ (Figure 2-1). In flies, the 2 genes flanking Dube $3 a$ are 

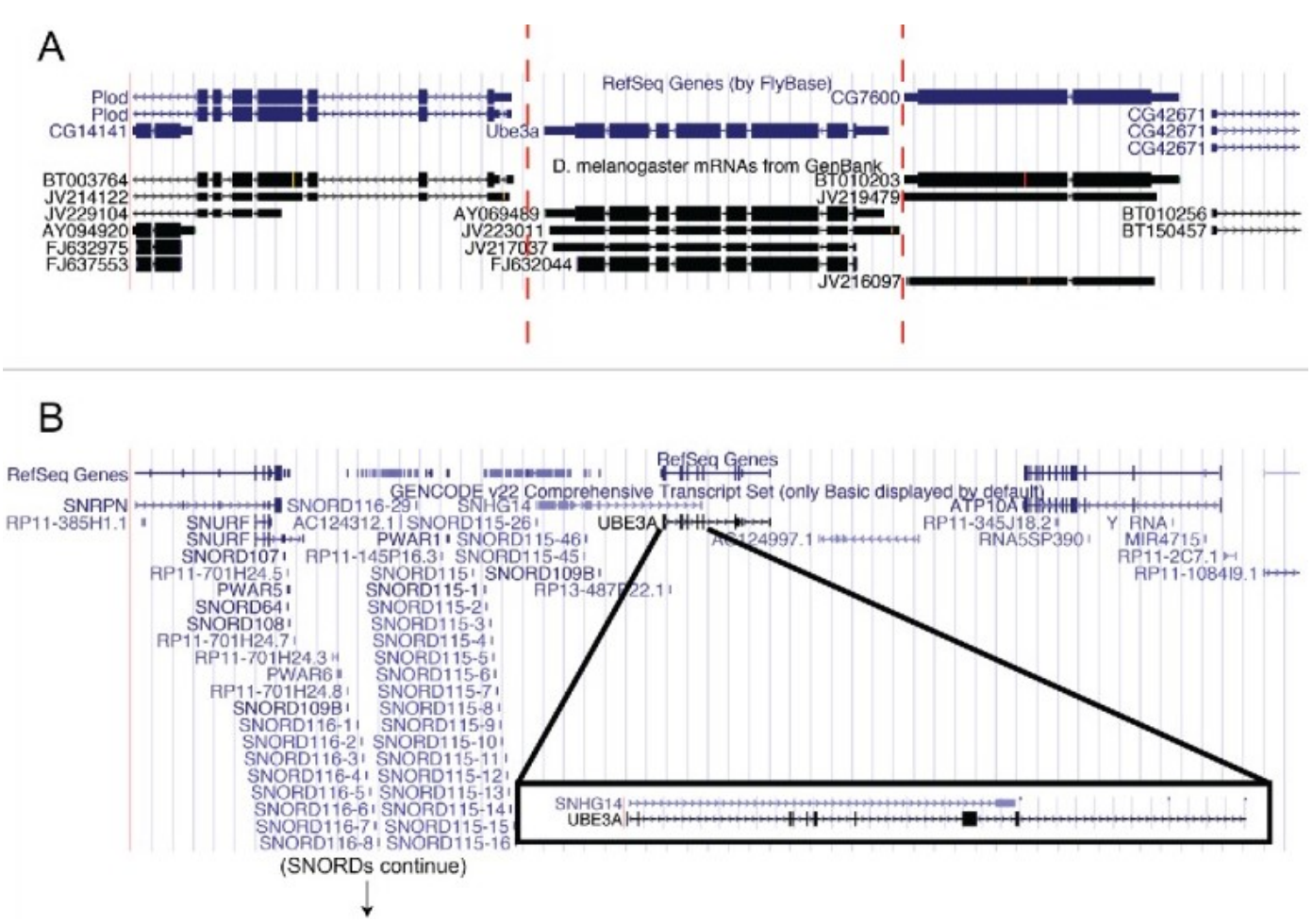

Figure 2-1. The genomic region surrounding $U B E 3 A$ is not syntenic between flies and humans.

Images from the UCSC genome browser (http://genome.ucsc.edu/) for the $10 \mathrm{~kb}$ region surrounding Dube $3 a$ in flies $(\mathbf{A})$ and $1 \mathrm{Mb}$ surrounding $U B E 3 A$ in humans $(\mathbf{B}$; note: the $S N O R D$ cluster has been truncated). In flies, there is no cluster of snoRNAs or the presence of $S N U R F / S N R P N$ upstream or downstream of Dube3a. There is also no RNA transcript that overlaps with Dube $3 a$ (A; red bars). In humans, note the presence of $S N H G 14(U B E 3 A-A S)$ that overlaps with $U B E 3 A$ transcript (B, boxed region). 
Plod and CG7600, which have human homologs located at 7q22.1 and 8q24.13, respectively. No antisense transcript has been reported to interfere with Dube3a expression at this locus (Figure 2-1A). In contrast, the human $S N H G 14$ (UBE3A-AS) transcript clearly overlaps with the $U B E 3 A$ transcript (Figure 2-1B). Based on these data, it appears unlikely that fly Dube $3 a$ is preferentially expressed from the maternal allele as the surrounding genomic region is not syntenic to the human $U B E 3 A$ region. There are no antisense transcripts detected across the Dube $3 a$ locus.

\section{Climbing Behavior is Not Dependent Upon Maternal Dube3a Expression}

Previous work demonstrated climbing deficiencies in homozygous Dube $3 a^{15 b}$ (Dube $3 a$ null) flies, as significantly fewer Dube $3 a^{15 b}$ flies climbed $3 \mathrm{~cm}$ within $3 \mathrm{~s}(\mathrm{Wu}$, Bolduc et al. 2008). Our experiments showed a significant effect of $D u b e 3 a^{15 b}$ allele on climbing ability (One-way ANOVA, $\mathrm{F}_{(3,20)}=6.99, P=0.0021$ ). Tukey's multiple comparisons test indicated that $D u b e 3 a^{15 b}$ homozygous flies took significantly longer to climb to a height of $4 \mathrm{~cm}$ compared to control $w^{1118}$ flies, confirming previous reports of climbing defects in homozygous Dube $3 a^{15 b}$ flies. Flies that inherited this $D u b e 3 a^{15 b}$ allele through either the paternal or maternal germline displayed no differences in climbing behavior from controls (Figure 2-2). These data indicate that a single copy of functional $D u b e 3 a$ of either paternal or maternal origin is sufficient for normal climbing behavior and provide evidence that Dube3a is neither imprinted nor preferentially expressed from the maternal allele.

\section{Synaptic Transmission Defects in $D u b e 3 a^{15 b}$ Mutants are Present Independent of Parent of Origin}

We previously demonstrated impaired synaptic transmission in $D u b e 3 a^{15 b}$ flies at the neuromuscular junction (Valdez, Scroggs et al. 2015). Here we investigate neuronal activity and synaptic transmission in photoreceptor neurons of the fly eye using ERGs. In the ERG signal, the "on" and "off" transients are indirect measures of synaptic transmission, while the photoreceptor potential is a measure of photoreceptor neuron depolarization (Ugur, Chen et al. 2016). Control $w^{1118}$ flies displayed robust on/off transients and large photoreceptor potentials (Figure 2-3, top trace). The Dube3a $a^{15 b}$ mutation significantly affected both on transients (One-way ANOVA, $\mathrm{F}_{(3,32)}=11.75, P \leq$ $0.0001)$ and off transients (One-way ANOVA, $\mathrm{F}_{(3,32)}=22.63, P \leq 0.0001$ ). Tukey's post hoc multiple comparisons testing indicated that the presence of the Dube $3 a^{15 b}$ allele (either homozygous or heterozygous) reduced both on and off transients regardless of the parent of origin of the mutant allele as compared to control flies. No significant differences were observed in on/off transients among $D u b e 3 a^{15 b}$ allele carrying flies (heterozygous or homozygous). We observed no effect of this mutation on photoreceptor potentials (One-way ANOVA, $\mathrm{F}_{(3,32)}=2.88, P=0.057$ ). Thus, the Dube $3 a^{15 b}$ allele appears to effect synaptic transmission components of the ERG signal without interfering with the photoreceptor neuron depolarization. Haploinsufficiency for Dube3a is also 


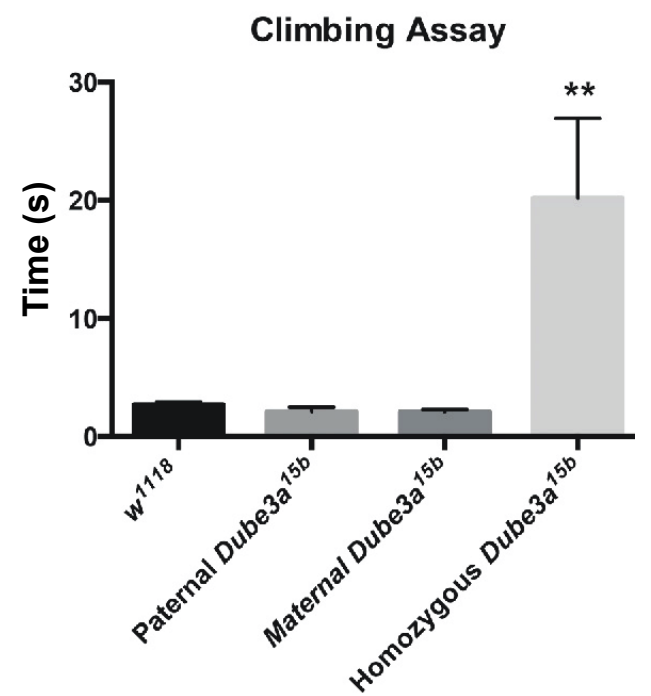

Figure 2-2. Only Dube3a $a^{15 b}$ homozygous LOF flies display climbing deficits. At 5 days of age, flies were tested for motor function. Homozygous Dube $3 a^{15 b}$ flies had significantly impaired climbing ability compared to control $w^{1118}$ flies $(\mathrm{P}=0.0021)$.

Paternally or maternally inherited $D u b e 3 a^{15 b}$ heterozygotes did not differ from controls in climbing ability. Data are presented as mean with standard error, $\mathrm{n}=6$ for all groups. 

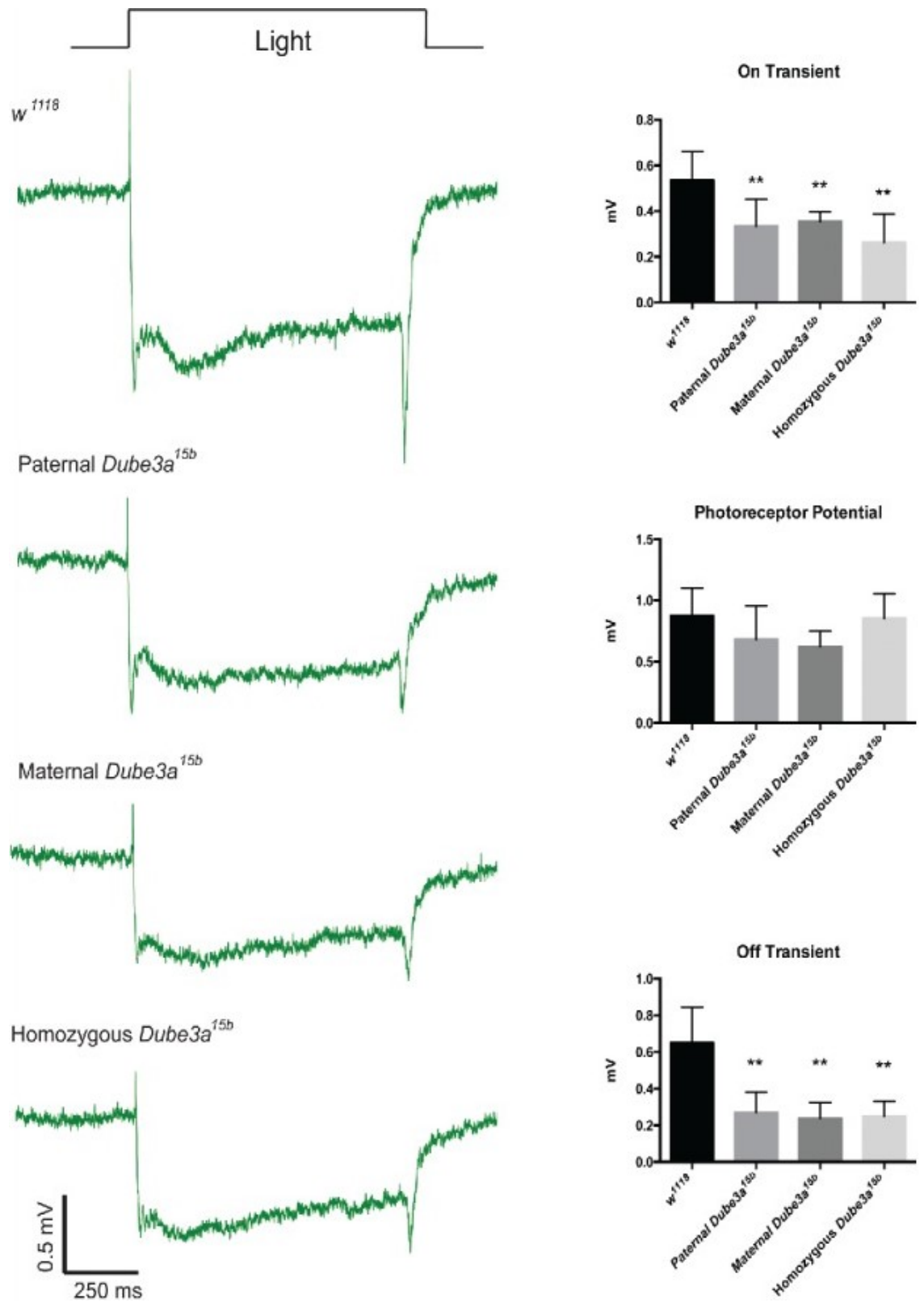

Figure 2-3. Heterozygous Dube $3 a^{15 b}$ flies have reduced on/off transients in the ERG signal independent of parent of inheritance.

Control $w^{1118}$ flies $(\mathrm{n}=12)$ display robust on/off transients that are significantly reduced in paternal $(\mathrm{n}=6)$, maternal $(\mathrm{n}=7)$, and homozygous $\operatorname{Dube}^{15 b}(\mathrm{n}=11)$ flies (On Transient, $\mathrm{P}<0.0001$; Off transient, $\mathrm{P}<0.0001)$. Note that the Dube $3 a^{15 b}$ mutation does not impair photoreceptor potentials. Data are presented as means with standard error. 
sufficient to decrease synaptic transmission regardless of the parental origin of the mutation, thus indicating that $D u b e 3 a$ is not preferentially expressed from either allele in the brain.

\section{Allele-specific Expression of Dube3a}

To investigate Dube $3 a$ allele specific expression at the molecular level, we performed reciprocal crosses with flies from the DGRP containing coding SNPs in Dube3a. Using the UCSC genome browser of the DGRP2 data (Mackay, Richards et al. 2012) located at dgrp2.gnets.ncsu.edu, we selected coding SNPs located in Dube3a. Three separate DGRP lines were used; RAL-239(G117T), RAL-313(G78T), and RAL517(C171A), which each contain coding SNPs in Dube3a as compared to the reference line RAL-21. Female RAL-239 crossed to male RAL-21 and female RAL-21 crossed to male RAL-239 showed 2 similarly sized ( $\sim 50 \%)$ peaks in the chromatogram at position 117 in the Dube3a cDNA sequence (Figure 2-4A). Similar results were observed between reciprocal crosses of RAL-313 crossed to RAL-21 at position 78 (Figure 2-4B), and RAL-517 and RAL-21 at position 171 (Figure 2-4C). These data align with our previous experiments and support the conclusion that Dube $3 a$ is biallelically expressed in the fly brain, and neither imprinted nor preferentially expressed from the maternal allele.

\section{Discussion}

Our behavioral and molecular analyses indicate that neural expression of Dube $3 a$ alleles is independent of parental origin. Using the same Dube $3 a^{15 b}$ allele as Wu et al., we confirmed climbing deficiency in Dube3a homozygous LOF flies (Wu, Bolduc et al. 2008). Here we demonstrated that paternally or maternally-deficient $D u b e 3 a^{15 b}$ flies do not display climbing difficulties, indicating that one functional copy of Dube $3 a$ is sufficient for normal motor function independent of parent of origin. Our lab previously demonstrated synaptic transmission deficits at the neuromuscular junction in Dube $3 a^{15 b}$ homozygous flies (Valdez, Scroggs et al. 2015), and we confirmed synaptic transmission deficits here using ERGs. In our previous work at the $3^{\text {rd }}$ instar neuromuscular junction, we reported that excitatory junction potentials decreased in amplitude faster in Dube $3 a$ LOF flies in comparison with controls (Valdez, Scroggs et al. 2015), possibly due to a depletion of synaptic vesicles in the readily releasable pool (Desai-Shah and Cooper 2009). Here we show reductions in on/off transients in the ERG signal, which may also be due to a depletion of synaptic vesicles. Regardless, on/off transients were reduced in Dube $3 a$ homozygous and reciprocal heterozygous Dube $3 a^{15 b}$ flies, supporting the conclusion that Dube $3 a$ is neither imprinted nor preferentially expressed and that Dube $3 a$ haploinsufficiency is sufficient to cause synaptic transmission defects. The strongest piece of evidence indicating that Dube $3 a$ is not imprinted in flies comes from sequencing several different wild-type alleles of Dube3a. Reciprocal crosses for 3 separate wild-type Dube $3 a$ alleles indicated similar, if not identical, expression of both maternally and paternally-inherited alleles. 
A

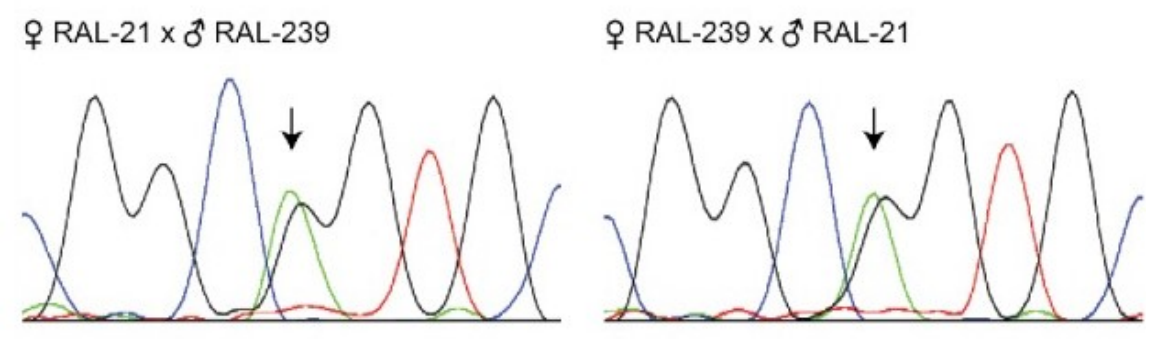

B

† RAL-21 $\times$ ठ̛ RAL-313

ㅇ RAL-313 $\times \sigma^{\top}$ RAL-21
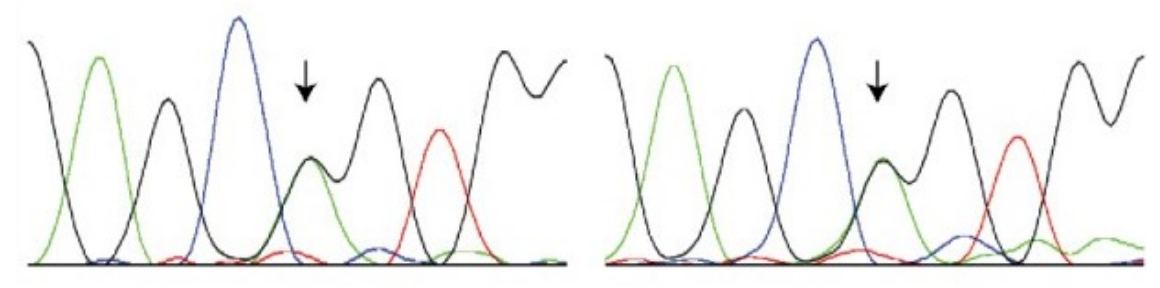

C

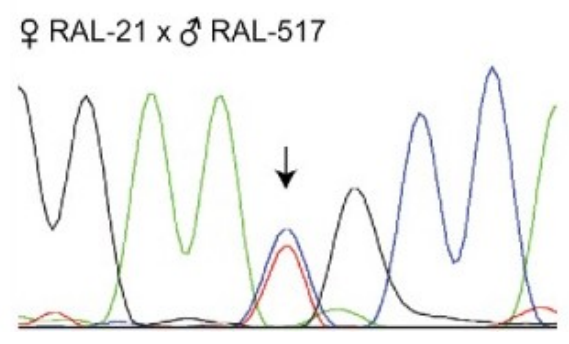

○ RAL-517 $\times \sigma^{\wedge}$ RAL-21

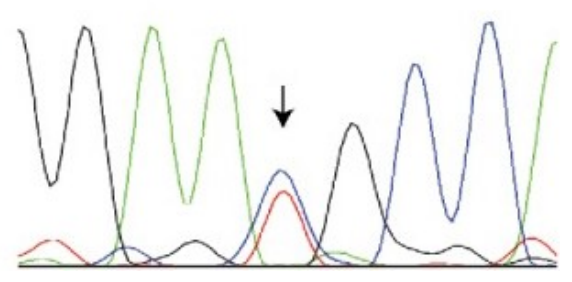

Figure 2-4. Sanger sequencing chromatograms of different wild-type Dube3a coding SNPs does not indicate imprinting or preferential allele specific expression. Total fly head RNA from reciprocal crosses with different coding SNPs present in Dube $3 a$ was DNase I treated and converted to cDNA. Sanger sequencing was performed on amplified cDNA using primers flanking the SNP to identify the expression of paternally or maternally inherited Dube $3 a$ alleles. In all alleles tested, we found similar expression levels of maternally and paternally inherited coding region alleles as indicated by 2 peaks at each SNP of the same height (arrows). 
In contrast to Chakraborty et al. (Chakraborty, Paul et al. 2015), we found no evidence that $D u b e 3 a$ is imprinted or preferentially expressed from the maternal allele in Drosophila melanogaster. On the other hand, our data is compatible with previously reported learning deficits in Dube3a maternally-deficient flies since we found that Dube3a haploinsufficiency results in ERG on/off transient deficiencies regardless of parental origin. As the $U B E 3 A$ imprinting mechanism in mature mammalian neurons is complex and similar PWS/AS-ICR or antisense transcripts have not been detected in Drosophila, our behavioral, electrophysiological, and Dube $3 a$ allele-specific expression data suggest that $D u b e 3 a$ is neither imprinted nor preferentially expressed in fruit flies. 


\section{CHAPTER 3. INVESTIGATING SYNERGISTIC INTERACTIONS BETWEEN DUBE3A AND HERC2}

\section{Introduction}

Autism spectrum disorder (ASD) has a prevalence of 1 in 59 (Baio, Wiggins et al. 2018) and the identification of causal genes has been limited. The heritability of ASD is estimated to be $83 \%$ (Sandin, Lichtenstein et al. 2017), indicating that ASD is highly heritable. However, causal genes have only been identified in approximately $25 \%$ of individuals and each gene mutation/variant is individually rare and only explains $1-2 \%$ of cases (Yoo 2015). Perturbation of multiple genes in the same biological pathway may be necessary to generate ASD phenotypes (Noh, Ponting et al. 2013), which may contribute to the lack of identified ASD genes. De novo copy number variations (CNVs) resulting from genomic duplications and deletions are prevalent in ASD (Sebat, Lakshmi et al. 2007, Pinto, Pagnamenta et al. 2010, Moreno-De-Luca, Sanders et al. 2013). CNVs harboring multiple genes known to interact with one another that cause elevated rates of ASD are, therefore, good candidates to identify genes contributing to ASD risk.

Recurrent de novo duplications of the 15q11.2-q13.1 region are associated with increased risk for ASD (Cook, Lindgren et al. 1997, Sanders, Ercan-Sencicek et al. 2011, Malhotra and Sebat 2012, Moreno-De-Luca, Sanders et al. 2013). Two genes within the $15 q$ duplication, ubiquitin protein ligase E3A (UBE3A), an E3 ubiquitin ligase involved in protein degradation and HECT and RLD domain containing E3 ubiquitin protein ligase 2 (HERC2), encode proteins that physically interact with one another. HERC2 stimulates the ubiquitin ligase activity of UBE3A, independent of its own ubiquitin ligase function, through an interaction between the RCC1-like domain 2 (RLD2) of HERC2 with the Nterminus of UBE3A (Kuhnle, Kogel et al. 2011, Galligan, Martinez-Noel et al. 2015). Individuals with loss-of-function point mutations in HERC2 present clinically with Angelman syndrome-like phenotypes (Puffenberger, Jinks et al. 2012, Harlalka, Baple et al. 2013, Morice-Picard, Benard et al. 2016), providing further evidence that UBE3A and HERC2 function in similar biological pathways. Aside from these in vitro biochemical studies the downstream molecular consequences of the interaction between UBE3A and HERC2 has not be thoroughly investigated.

To investigate the interaction between UBE3A and HERC2, and to determine if they act synergistically to cause Dup15q-associated phenotypes including ASD, we turned to Drosophila and the GAL4/UAS expression system (Duffy 2002). In this chapter, we present data from flies overexpressing Dube3a alone, HERC2 alone (the fly $U B E 3 A$ and $H E R C 2$ orthologs, respectively), or both genes simultaneously to reveal the contributions of each gene alone and synergistically to several behavioral phenotypes. By taking a whole organism approach we are able to assay the interaction between Dube3a and HERC2 in a fully intact organism which accounts for developmental effects and interactions among different cell types and allows for the analysis of complex behaviors that are not possible to investigate in vitro. 


\section{Methods}

\section{Fly Stocks}

Flies were raised on standard corn meal media (Blooming Drosophila Stock Center) and maintained on a 12-h light/dark cycle at $25^{\circ} \mathrm{C}$. The UAS-HERC2 enhancer piracy line (BDSC \# 33296) and PDF-GAL4 (BDSC \#6900) were obtained from the Bloomington Drosophila Stock Center (Bloomington, IN). The UAS-Dube3a line was described previously (Reiter, Seagroves et al. 2006), and c155-GAL4 was generously donated by Dr. Hugo Bellen. Since the $c 155-G A L 4$ driver and the HERC2 enhancer piracy element are both present on the $\mathrm{X}$ chromosome, all experiments were performed on female flies.

\section{RLD2 Domain Comparison Between Human and Fly HERC2}

Amino acid sequences of the RLD2 domain from human and fly HERC2 were compared using the Clustal Omega multiple sequence alignment tool (Sievers, Wilm et al. 2011). The RLD2 domain of human HERC2 consists of amino acids 2958-3326 (UniProt Accession \#O95714) and the RLD2 domain of fly HERC2 consists of amino acids 2985-3352 (UniProt Accession \#Q9VR91).

\section{Quantitative Real-time Polymerase Chain Reaction (qRT-PCR)}

Total head mRNA was extracted in TRI Reagent (Applied Biosystems) and RNA was extracted and purified using the Directzol RNA Miniprep Plus kit (Zymo Research Corp) according to the manufacturer's instructions. Purity and concentration were verified using a NanoDrop spectrophotometer (ThermoFisher Scientific). Assays were performed in triplicate on a Roche LC480 thermocycler with the following cycling parameters: $95^{\circ} \mathrm{C}$ for $5 \mathrm{~m}$ followed by 40 cycles of $95^{\circ} \mathrm{C}$ for $10 \mathrm{~s}, 60^{\circ} \mathrm{C}$ for $30 \mathrm{~s}$, and $72^{\circ} \mathrm{C}$ for $10 \mathrm{~s}$ during which fluorescence was measured. Crossing point $(\mathrm{Cp})$ values were calculated with the absolute quantification algorithm (Roche), and average $\mathrm{Cp}$ values for three technical replicates were calculated, normalized to TATA-binding protein (tbp), and fold change was calculated with the $(2)^{-\Delta \Delta C p}$ method. The following primers were used with probe \#25 from the Universal Probe Library (UPL) for HERC2: F 5'TCACCTGGTTCTGGGAGGT-3', R 5'-CGAACCGCAGGAATAGAGAG-3'. For tbp, the following primers were used with UPL probe \#109: F: 5'ACAGGGGCAAAGAGTGAGG-3', R 5'-CTTAAAGTCGAGGAACTTTGCAG-3'.

\section{Quantitative Western Blot Analysis}

Western blots were performed as previously described (Hope, LeDoux et al. 2017). Fly heads were removed and homogenized in radio-immunoprecipitation assay 
(RIPA) buffer with complete protease inhibitor (Roche), and $20 \mu \mathrm{g}$ of fly head protein was loaded into each lane of a $1.5 \mathrm{~mm}$ NuPAGE Bis-Tris 4-12\% gradient gel (Invitrogen) and transferred to a PVDF membrane (Millipore). Membranes were blocked with 5\% milk, 3\% bovine serum albumin, and $0.1 \%$ Tween-20 in phosphate buffered saline. Primary antibodies used were rat $\alpha$-Dube3a at 1:4000 (gift from Dr. Janice Fischer) and goat $\alpha$-GAPDH at 1:5000 (IMGENEX Cat\# IMG-3073). $\alpha$-goat and $\alpha$-rat, infrared secondary antibodies were used at a dilution of 1:15,000. Blots were imaged using the Odyssey Infrared Imaging System (Li-Cor), analyzed using Image Studio Lite software (Li-Cor), and all lanes were normalized to GAPDH.

\section{Social Space Assay}

Flies 1-3 days of age were collected under $\mathrm{CO}_{2}$ anesthesia and allowed two days to recover prior to testing. For testing, flies were briefly immobilized by placing on ice for 2 minutes before transferring into the triangle testing apparatus described previously (Simon, Chou et al. 2012). Due to reported climbing defects observed in $c 155>$ Dube $3 a$ flies (Wu, Bolduc et al. 2008), we performed these assays with the triangles in a horizontal position. After 30 minutes in the triangle, an image was captured and ImageJ was used to calculate the nearest neighbor distance between each fly.

\section{Neuromuscular Junction Immunohistochemistry and Image Analysis}

NMJ analysis was performed on larvae 5 days post egg laying. Female larvae were selected by the absence of the developing gonad and dissected in phosphate buffered saline. Larvae were fixed for 15 minutes in $4 \%$ formaldehyde at room temperature. Larval fillets were blocked and permeabilized with PBS containing 1\% bovine serum albumin and $0.01 \%$ Triton $X-100$, followed by incubation with $\alpha$-discs large (DSHB, 4F3) at 1:100. Goat $\alpha$-mouse 594 secondary antibody (Invitrogen A11005) was used at 1:500. NMJs innervating muscles 6/7 from hemisegment A3 were imaged using a laser-scanning confocal microscope (Zeiss LSM 710). Z-stacks through each NMJ were obtained using a $63 \mathrm{X}$ oil-immersion objective at $1024 \times 1024$ resolution. NMJ length was calculated using Image $(\mathrm{NIH})$.

\section{Activity Monitoring and Circadian Rhythm Analysis}

Female flies were collected on the day of eclosion and entrained for 2 days under 12-hour light/dark cycle. On day 3, individual flies were loaded into glass tubes containing standard Drosophila corn meal media and stoppered with a small cotton plug. Locomotor activity was monitored using the Drosophila Activity Monitoring System (DAMS, Trikinetics). Activity was measured for 3 days during light/dark cycles, followed by 7 days of activity monitoring during complete darkness (dark/dark) to measure the freerunning period. 
Data were visualized and analyzed using the ActogramJ plugin for ImageJ (Schmid, Helfrich-Forster et al. 2011). Actograms were averaged for each genotype ( $\mathrm{n} \geq$ 4 per genotype), and period length was calculated using the ActogramJ built in Chisquare analysis with a window of 1000-2000 minutes to identify cycle length.

\section{Results}

\section{Human and Fly HERC2 Are Highly Conserved Within the RLD2 Domain}

The human HERC2 protein is 4834 amino acids in length and contains 8 distinct domains. Similarly, the Drosophila melanogaster HERC2 protein is 4912 amino acids in length and contains many of the same domains as human HERC2, including the RLD2 domain (Figure 3-1A). Across the whole protein, human and fly HERC2 are 46.1\% identical, however within the RLD2 domains they share $74.46 \%$ identity (Figure 3-1B). Based on the high conservation between RLD2 domains of human and Drosophila HERC2, we hypothesize that the RLD2 domain of Drosophila HERC2 should be able to stimulate Dube3a activity as previously shown in vitro for human HERC2 and UBE3A (Kuhnle, Kogel et al. 2011).

\section{Drosophila HERC2 Stimulates the Ubiquitin Ligase Activity of Dube3a}

In order to overexpress Drosophila $H E R C 2$ we obtained an enhancer piracy line that contains a P-element with a UAS sequence upstream of HERC2 (BDSC \#33296, referred to as UAS-HERC2). Quantitative real-time polymerase chain reaction (qRTPCR) was performed on total head mRNA from $c 155>H E R C 2$ flies. Compared to control c155-GAL4 alone, the $c 155>H E R C 2$ line had a $5.6 \pm 1.35$-fold elevation of HERC2 transcript, indicating that the UAS-HERC2 line does indeed overexpress HERC2 transcript when crossed to $c 155-$ GAL4.

Next, we tested whether fly HERC2 is capable of stimulating the ubiquitin ligase activity of Dube3a in vivo. There are many potential UBE3A substrates published, but no one substrate has been accepted in the field as a hallmark of UBE3A ubiquitin ligase function (LaSalle, Reiter et al. 2015). However, it is well-established that UBE3A autoubiquitinates itself in trans (Nuber, Schwarz et al. 1998), making Dube3a a viable target to test for Dube3a activity. We performed western blots for Dube3a levels in flies overexpressing Dube $3 a$ alone, HERC2 alone, or both Dube $3 a$ and HERC2 simultaneously with the pan-neuronal GAL4 driver c155-GAL4. Dube3a levels were significantly dependent upon genotype (Figure 3-2A, B, One-way ANOVA, $\mathrm{F}_{(3,8)}=228$, $\mathrm{p}<0.0001)$, and Tukey's multiple comparisons test $(\alpha<0.05)$ revealed that $c 155>$ Dube3a flies had elevated levels of Dube3a compared to $c 155-$ GAL4 alone flies, and this increase in Dube3a was reduced upon simultaneous overexpression of HERC2. Additionally, overexpression of $H E R C 2$ alone significantly reduced levels of Dube3a 
A

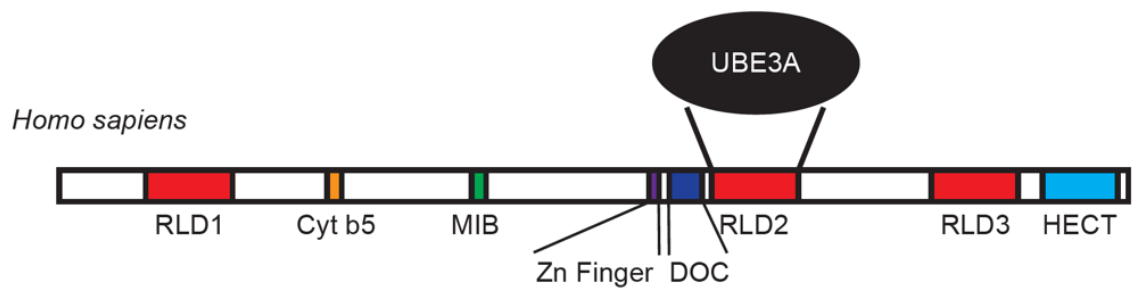

Drosophila melanogaster

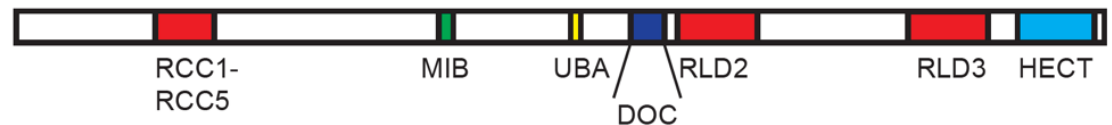

B

\begin{tabular}{|c|c|}
\hline Human_RLD2 & RTKVFVWGLNDKDQLGGLKGSKIKVPSFSETLSALNVVQVAGGSKSLFAVTVEGKVYACG \\
\hline Drosophila_RLD2 & $\begin{array}{l}\text { PCTVMVWGLNDKEQLGGLKGSKVKVPTFSQTISRLRPIHIAGGSKSLFIVSQDGKVYACG } \\
*^{*}: * * * * * * * * * * * * * * * * * *: * *: *:^{*} \cdot::: * * * * * * * *:: * * * * * *\end{array}$ \\
\hline $\begin{array}{l}\text { Human_RLD2 } \\
\text { Drosophila_RLD2 }\end{array}$ & 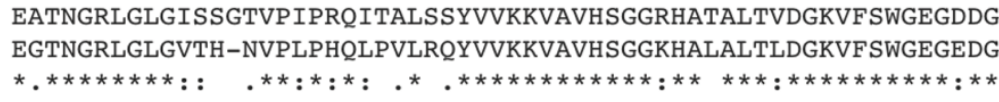 \\
\hline $\begin{array}{l}\text { Human_RLD2 } \\
\text { Drosophila_RLD2 }\end{array}$ & $\begin{array}{l}\text { KLGHFSRMNCDKPRLIEALKTKRIRDIACGSSHSAALTSSGELYTWGLGEYGRLGHGDNT } \\
\text { KLGHGNRTTLDKPRLVEALRAKKIRDVACGSSHSAAISSOGELYTWGLGEYGRLGHGDNT } \\
\star * * * * * * * * * * * *:: *: * * * * * * * * * *:: * * * * * * * * * * * * * * * * *\end{array}$ \\
\hline $\begin{array}{l}\text { Human_RLD2 } \\
\text { Drosophila_RLD2 }\end{array}$ & $\begin{array}{l}\text { TQLKPKMVKVLLGHRVIQVACGSRDAQTLALTDEGLVFSWGDGDFGKLGRGGSEGCNIPQ } \\
\text { TQLKPKLVTALAGRRVVQVACGSRDAQTLALTEDGAVFSWGDGDFGKLGRGGSEGSDTPH } \\
* * * * * * * . * * *: * *: * * * * * * * * * * * *:: * * * * * * * * * * * * * * * * *: * *\end{array}$ \\
\hline $\begin{array}{l}\text { Human_RLD2 } \\
\text { Drosophila_RLD2 }\end{array}$ & $\begin{array}{l}\text { NIERLNGQGVCQIECGAOF SLALTKSGVVWTWGKGDYFRLGHGSDVHVRKPQVVEGLRGK } \\
\text { EIERLSGIGVVQIECGAQFSLALTRAGEVWTWGKGDYYRLGHGGDQHVRKPQPIGGLRGR } \\
: * * * * * * * * * * * * * * * * *: * * * * * * * * * * * * * * * * * * \text { : } * * * *\end{array}$ \\
\hline $\begin{array}{l}\text { Human_RLD2 } \\
\text { Drosophila_RLD2 }\end{array}$ & $\begin{array}{l}\text { KIVHVAVGALHCLAVTDSGQVYAWGDNDHGQQGNGTTTVNRKPTLVQGLEGQKITRVACG } \\
\text { RVIHVAVGALHCLAVTDAGQVYAWGDNDHGQQGSGNTFVNKKPALVIGLDAVFVNRVACG } \\
::: * * * * * * * * * * *: * * * * * * * * * * * * * * * * * * * * * * \text { I }: * * * *\end{array}$ \\
\hline $\begin{array}{l}\text { Human_RLD2 } \\
\text { Drosophila_RLD2 }\end{array}$ & $\begin{array}{l}\text { SSHSVAWTT } \\
\text { SSHSIAWGL } \\
\star * * *: * *\end{array}$ \\
\hline
\end{tabular}

Figure 3-1. Human and Drosophila HERC2 proteins are homologous.

A) Domains from human (Homo sapiens, top) and fly (Drosophila melanogaster, bottom) are indicated across the HERC2 protein. The interaction domain between human HERC2 and UBE3A is called RLD2. B) Amino acid sequence alignment from the human and Drosophila RLD2 domain. The RLD2 domain that interacts with UBE3A shares a high degree of identity at $74.46 \%$. 
A

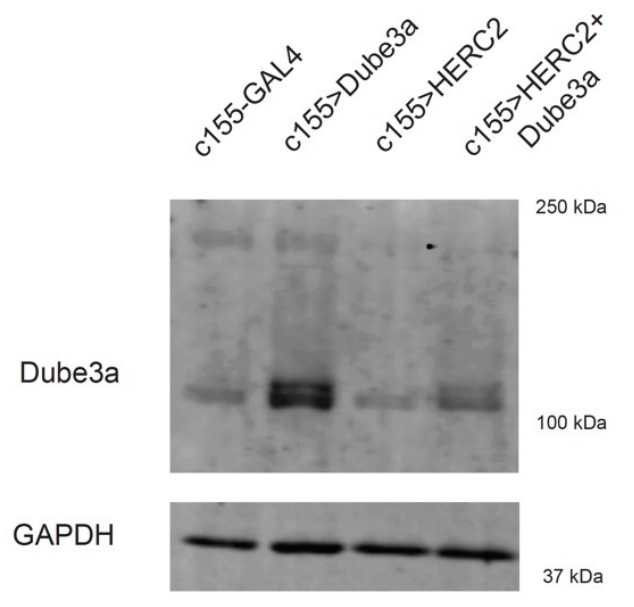

B

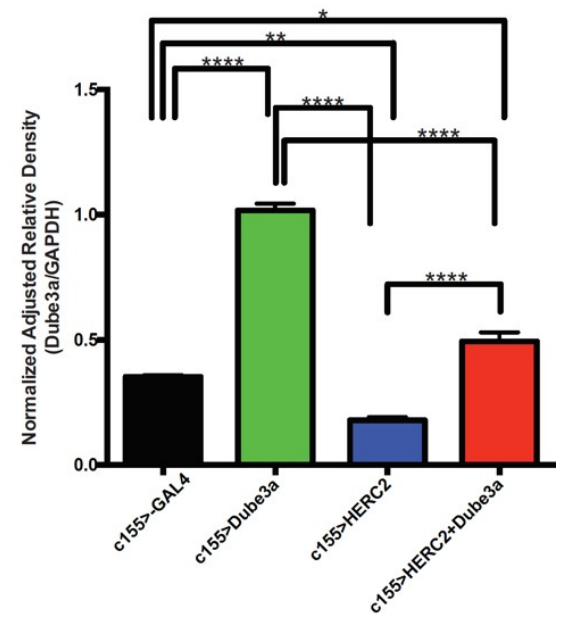

Figure 3-2. Drosophila HERC2 stimulates the ubiquitin ligase activity of Dube3a. UBE3A/Dube3a autoubiquitinates itself so Dube3a levels can be used as a measure of Dube3a activity. A) Western blot for levels of Dube3a protein following overexpression in neurons of Dube $3 a$ alone, HERC2 alone, or both HERC2+Dube $3 a$ with $c 155-$ GAL4. B) Quantification of western blot signals indicates that Dube3a levels were elevated in $c 155>$ Dube $3 a$ as predicted, however upon simultaneous overexpression of $H E R C 2$ we observed reduced levels of Dube3a protein (green bar vs. red bar). Additionally, we observed reduced Dube3a levels of endogenous Dube3a when only overexpressing $H E R C 2$ alone (black bar vs. blue bar). Data are presented as mean $\pm \mathrm{SEM}, \mathrm{n}=3$ for all samples. $*=p<0.05, * *=p<0.01, * * *=p<0.005$, and $* * * *=p<0.001$. 
compared to control c155-GAL4, indicating that overexpression of HERC2 alone reduces levels of endogenous Dube3a. These data suggest that Drosophila HERC2 is able to stimulate the ubiquitin ligase of Dube3a in vivo, similar to what occurs in vitro with mammalian UBE3A/HERC2.

\section{Simultaneous Overexpression of HERC2 and Dube3a Increases Social Spacing}

In order to assay ASD-like behavior in flies we performed two assays to measure social interaction and repetitive/restrictive interests. First, we used a previously published assay that measures social interaction in which flies are placed in a triangular shaped apparatus (Figure 3-3A) and allowed to equilibrate their spacing among each other within a group (Simon et al., 2012). While this assay is typically performed with the triangle in a vertical orientation, we performed the assay in a horizontal orientation to avoid confounds from known climbing defects observed in c155>Dube3a flies (Wu, Bolduc et al. 2008). We observed a significant effect of genotype on the average social space for each genotype (Figure 3-3B, One-way ANOVA, $\mathrm{F}_{(3,298)}=2.69, \mathrm{p}<0.05$ ). A Tukey's multiple comparisons test $(\alpha<0.05)$ indicated that the only genotype significantly different from controls was $c 155>H E R C 2+D u b e 3 a$. Next, we performed a grooming assay to measure repetitive/restrictive interests. Flies were placed into a chamber and the time spent grooming during the 5-minute assay period was recorded. We observed a significant effect of genotype on time spent grooming (One-way ANOVA, $\left.\mathrm{F}_{(3,20)}=12.78, \mathrm{p}<0.0001\right)$, and $c 155>$ HERC2 flies displayed a significant increase total grooming time (Tukey's multiple comparisons test, $\alpha<0.005$ ). These data suggest a synergistic interaction between Dube3a and HERC2 in generating defects in social interaction and that elevated levels of only HERC2 impact repetitive/restrictive behaviors.

\section{Overexpression of $\mathrm{HERC} 2$ and Dube3a Impairs Synaptic Morphology at the Neuromuscular Junction}

The Drosophila neuromuscular junction (NMJ) has been used successfully to dissect how multiple genes located within a CNV contribute to disease pathology (Grice, Liu et al. 2015). Here, we took a similar approach and upon visualization of the NMJ with $\alpha$-DLG staining we observed a significant effect of genotype on NMJ length (Figure 3-4A, B, One-way ANOVA, $\left.\mathrm{F}_{(3,21)}=8.79, \mathrm{p}<0.0001\right)$. Tukey's multiple comparisons test $(\alpha<0.05)$ indicated that overexpression of HERC2 alone or Dube3a alone did not significantly affect NMJ morphology. However, simultaneous overexpression of both HERC2 and Dube3a resulted in significantly reduced NMJ length. These data indicate that HERC2 and Dube3a act synergistically to influence synaptic phenotypes, while overexpression of each gene alone does not result in appreciable differences at the NMJ. 
A

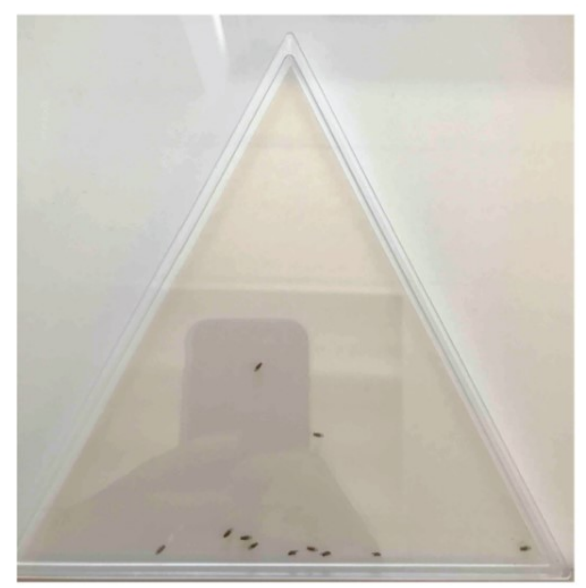

B

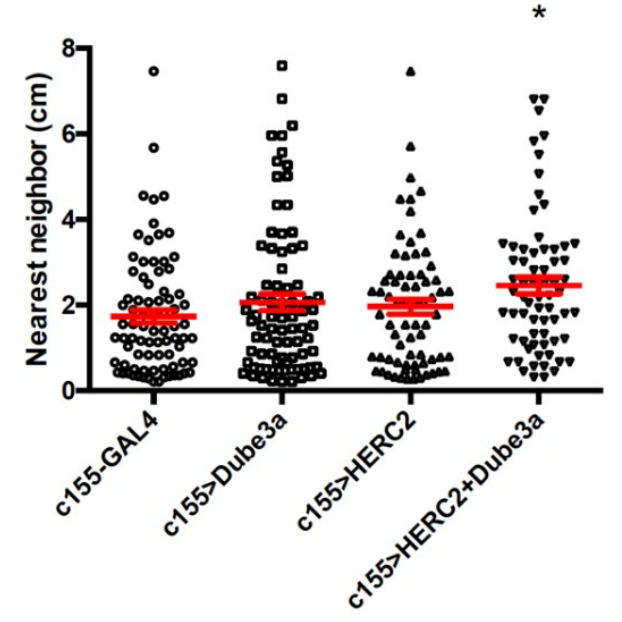

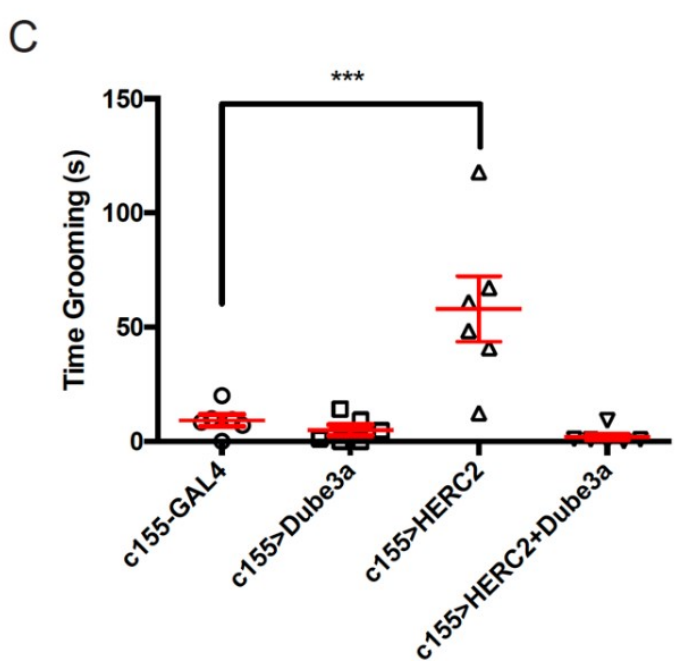

Figure 3-3. Simultaneous overexpression of HERC2 and Dube3a increases social spacing.

To investigate how Dube $3 a$ and $H E R C 2$ contribute to ASD-like behaviors we used a social space assay and a grooming assay to measure the social interaction and repetitive behavior components of ASD. A) Image of the triangle apparatus used to measure social spacing in flies. B) Average nearest neighbor for each genotype of flies in the social space assay. The average social space was significantly increased in $c 155>$ HERC2 + Dube $3 a$ flies compared to control c155-GAL4 alone flies (One-way ANOVA, $\mathrm{p}<0.05, \mathrm{n} \geq 68$ per genotype). C) Average time spent grooming per genotype during the 5-minute grooming assay. Grooming time was significantly increased in $c 155>$ HERC2 flies compared to all other groups (One-way ANOVA, $\mathrm{p}<0.001, \mathrm{n}=6$ per genotype). Data are presented as mean \pm SEM. 
A

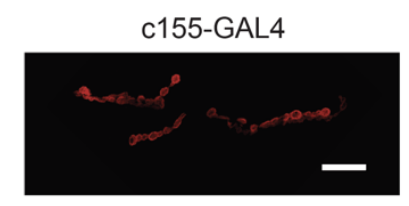

c155>HERC2

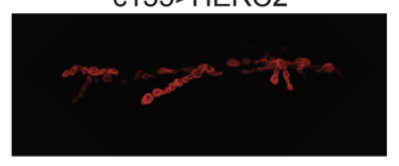

c155>Dube3a

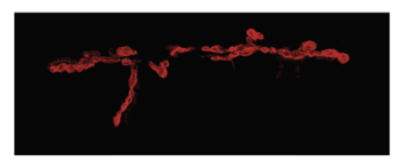

c155>HERC2+Dube3a

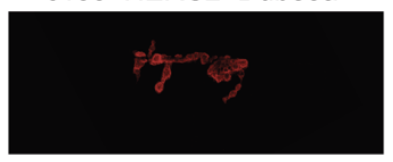

B

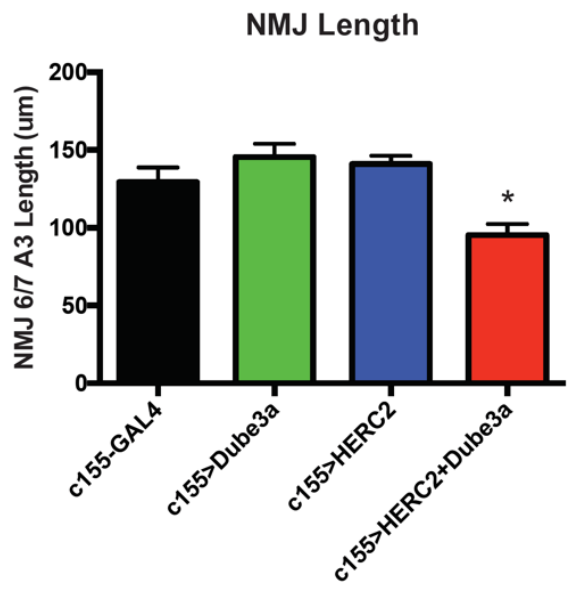

Figure 3-4. Overexpression of HERC2 and Dube3a impairs synaptic morphology at the neuromuscular junction.

A) Disks large (DLG) staining at the neuromuscular junction (NMJ) of segment A3, muscles 6/7 in $3^{\text {rd }}$ instar larva (scale bar is $25 \mu \mathrm{m}$. B) Quantification of NMJ length for each genotype. $c 155>H E R C 2+D u b e 3 a$ NMJs displayed significantly reduced NMJ length compared to control NMJs (One-way ANOVA, $\mathrm{p}<0.001, \mathrm{n}=6$ per genotype). 


\section{Co-overexpression of $H E R C 2$ and $U B E 3 A$ in PDF Neurons Impairs Circadian Rhythms}

Individuals with Dup15q often suffer from sleep problems and the AS deletion is associated with defects in BMAL1, a protein known to regulate circadian rhythm (Gossan, Zhang et al. 2014, Shi, Bichell et al. 2015). Here we investigated whether elevated levels of HERC2 or Dube $3 a$ impaired circadian rhythms in Drosophila. In these experiments we restricted overexpression to pigment dispersing factor $(P D F)$ positive neurons using the $P D F$-GAL4 driver since these cells are known to critically regulate circadian rhythms in flies (Renn, Park et al. 1999). Flies were entrained to a 12-hour light/dark cycle for 3 days and subsequently placed in complete darkness to monitor their freerunning period. Control PDF-GAL4 alone flies exhibited robust circadian rhythms in the light/dark cycle and the dark/dark cycle, with period lengths averaging 1440 minutes and 1410 minutes, respectively (Figure 3-5A, B). $P D F>D u b e 3 a$ flies presented a robust period length averaging 1440 minutes in both the light/dark cycle and in the dark/dark cycles, with no shortening of the freerunning period observed (Figure 3-5B).

$P D F>H E R C 2$ flies displayed similar periods to the control group, with periods of 1440 minutes in length in the light/dark cycle and periods of 1410 minutes in the dark/dark cycle (Figure 3-5B). In $P D F>H E R C 2+D u b e 3 a$ flies, periods were of similar length compared to the control group, averaging 1440 minutes in length, however in the dark/dark cycle no significant periodicity was detected (Figure 3-5B). These data suggest that simultaneous co-overexpression of both HERC2 and Dube 3 a interact synergistically to significantly alter circadian rhythms in Drosophila during the dark/dark freerunning period.

\section{Discussion}

Here, we investigated the interaction between Dube3a and HERC2 in vivo and the role that neuronal overexpression of each gene individually or simultaneously plays in generating phenotypes associated with Dup15q syndrome. Drosophila and human HERC2 proteins share a high degree of sequence identity and the data presented here suggests that Drosophila HERC2 increases the ubiquitin ligase function of Dube3a in vivo, just as predicted by in vitro studies measuring UBE3A activity in the presence of HERC2 or the HERC2 RLD2 domain (Kuhnle, Kogel et al. 2011). In measures of ASDlike behavior, simultaneous overexpression of both Dube $3 a$ and HERC2 increased social spacing, while overexpression of HERC2 alone increased the amount of time flies spent grooming. Simultaneous overexpression of Dube $3 a$ and $H E R C 2$ altered synaptic morphology at the NMJ, and overexpression of both genes in $P D F+$ neurons in the Drosophila brain altered the freerunning period in circadian rhythm analysis. Taken together, these data suggest a complex interplay and synergistic interactions in vivo between UBE3A and HERC2 in generating Dup15q phenotypes.

Our finding that elevated HERC2 reduces Dube3a levels are consistent with experiments in in vitro studies in which HERC2 physically interacts with and stimulates 
A
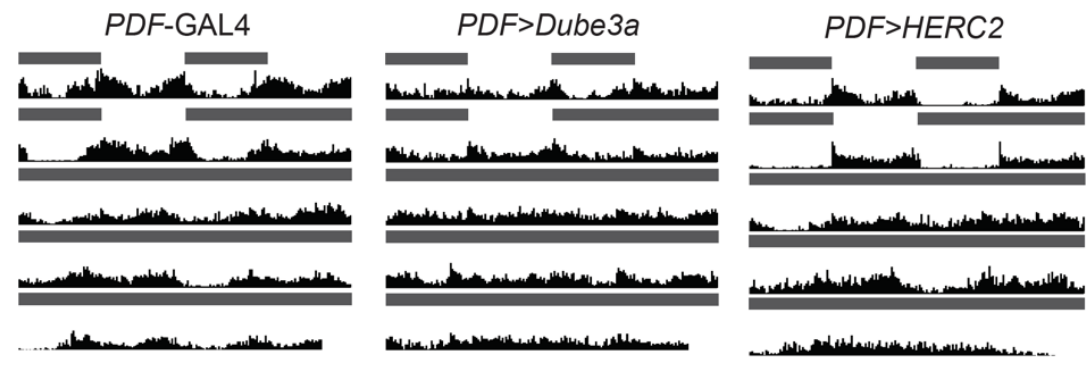

PDF>HERC2+Dube3a

B
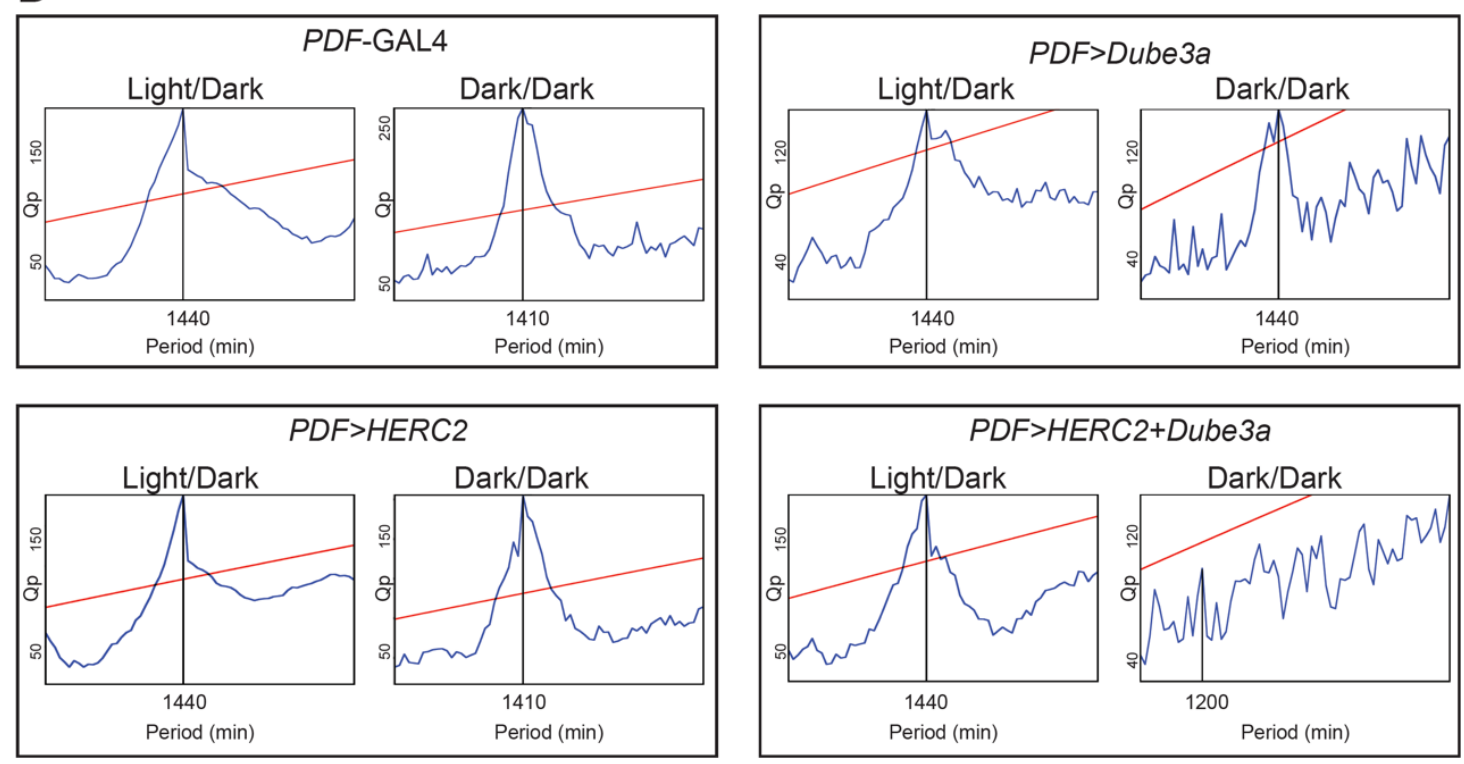

Figure 3-5. Simultaneous $H E R C 2$ and Dube3a overexpression impairs circadian rhythms.

A) Average activity plots ( $\mathrm{n} \geq 4$ per genotype) of control flies ( $P D F$-GAL4) or flies overexpressing Dube3a (PDF $>$ Dube3a), HERC2 (PDF $>$ HERC2), or both HERC2 and Dube3a $(P D F>H E R C 2+D u b e 3 a)$ in PDF+ neurons. Black horizontal bars indicate periods of darkness. Lights cycled on/off on a 12-hour period for 3 days, followed by 7 days of complete darkness. B) Period length analysis for average activity across each genotype in light/dark cycles and dark/dark cycles. In $P D F>H E R C 2+D u b e 3 a$, periodicity was completely abolished during the dark/dark period. 
the ubiquitin ligase activity of UBE3A (Kuhnle, Kogel et al. 2011). In both $c 155>H E R C 2$ and $c 155>H E R C 2+D u b e 3 a$ compared to their respective controls we observed reduced levels of Dube3a protein, suggesting increased autoubiquitination of Dube3a through elevated HERC2 levels. HERC2 consists of multiple protein domains, yet the RLD2 domain specifically is responsible for binding to and stimulating UBE3A, and increased activity of UBE3A is independent of the catalytic HECT domain of HERC2 (Kuhnle, Kogel et al. 2011). Human and Drosophila HERC2 proteins share a high degree of sequence conservation, especially within the RLD2 domain. While it is unlikely that the HECT domain of HERC2 is contributing to the increase in Dube3a activity, further experiments, such as expressing only the RLD2 domain of Drosophila HERC2 protein, should be conducted to establish that the in vitro activity paradigm holds in vivo under multiple conditions.

The NMJ morphology defects that we observed upon simultaneous overexpression of Dube $3 a$ and HERC2 are consistent with other studies using the Drosophila NMJ to identify causal genes in ASD-associated CNVs. For example, in cases of de novo CNV loss of chromosome $3 \mathrm{dlg}$ and pak double mutants (fly orthologs to genes located in the chromosome $3 \mathrm{CNV}$ ) displayed alterations in NMJ morphology, while single mutants displayed no NMJ phenotype (Grice, Liu et al. 2015). Copy number loss or gain of 16p11.2, a region containing approximately 30 genes, consistently generates an ASD phenotype and may account for up to 1\% of ASD cases (Marshall, Noor et al. 2008, Weiss, Shen et al. 2008), and CNVs that impact multiple genes are estimated to be found in up to $10 \%$ of the ASD population (Shishido, Aleksic et al. 2014). Sequencing known ASD genes from individuals in the Autism Clinical and Genetic Resources in China (ACGC) revealed that individuals carrying multiple de novo mutations in known ASD genes had more severe ASD phenotypes while parents carrying only a single variant were relatively unaffected (Guo, Wang et al. 2018). Our data fits into the two-hit, multifactorial model of ASD where altered function or expression of multiple genes confer increased ASD risk.

Consistent with previous reports, data presented here indicate that Dube $3 a$ is a regulator of Drosophila circadian rhythms. UBE3A ubiquitinates the key clock protein BMAL1 (cycle in Drosophila), and overexpression of Dube $3 a$ in PDF+ neurons caused impaired rhythms during dark/dark cycles in previous studies (Gossan, Zhang et al. 2014). In our experiments $P D F>D u b e 3 a$ flies remained rhythmic, however period length was altered. It is possible that our line expressed Dube $3 a$ at lower levels, resulting in a reduced phenotype. In line with the hypothesis that HERC2 protein stimulates the ubiquitin ligase function of Dube3a, we observed a complete lack of rhythmicity in $P D F>H E R C 2+D u b e 3 a$ flies indicating that HERC2 stimulates Dube3a activity. Sleep disturbances have been reported in Dup15q, particularly in cases of interstitial duplications (Urraca, Cleary et al. 2013), and our data implies that the interaction between UBE3A and HERC2 may underlie this aspect of Dup15q.

In conclusion, we used biochemical and behavioral assays to probe the interaction between Dube3a and HERC2 in vivo for the first time. We found that in some instances HERC2 elevation is the predominant driver of the observed phenotype, yet in other 
instances the synergistic interaction between HERC2 and Dube3a resulted in the phenotype. Our results highlight the use of Drosophila for investigating how multiple genes within a CNV may be contributing to disease pathology and suggest that the interaction between UBE3A and HERC2 is important in generating the phenotypes associated with Dup15q syndrome. 


\section{CHAPTER 4. GLIAL OVEREXPRESSION OF DUBE3A CAUSES SEIZURES AND SYNAPTIC IMPAIRMENTS IN DROSOPHILA CONCOMITANT WITH DOWNREGULATION OF THE NA ${ }^{+} / \mathrm{K}^{+}$PUMP ATP-ALPHA ${ }^{2}$}

\section{Introduction}

Duplication $15 q$ syndrome (Dup15q) is caused by either interstitial or isodicentric duplications of the chromosomal region 15q11.2-q13 .1. Dup15q is characterized by autism, developmental delay, mental retardation, hypotonia, and epilepsy. The most devastating feature of Dup15q is difficult to control seizures (Finucane, Lusk et al. 2016). Approximately $65 \%$ of isodicentric Dup $15 q$ individuals have seizures that often begin as infantile spasms that progress to Lennox-Gastaut type syndrome (Battaglia 2008, Finucane, Lusk et al. 2016). Poorly controlled epilepsy severely impacts the quality of life of both affected individuals and their caregivers. Current treatment options for Dup15q-associated epilepsy are often ineffective. GABAergic promoting antiepileptics are typically ineffective while broad-spectrum antiepileptic medications such as valproic acid and rufinamide provide some relief (Conant, Finucane et al. 2014). Elevated levels of $U B E 3 A$ in neurons or duplication of a cluster of GABA receptors (GABRA5, GABRB3, and $G A B R G 3$ ) located within Chr 15q11.2-q13.1 have been proposed as the etiology of Dup15q epilepsy, although 15q copy numbers do not correlate with GABA receptor transcript levels (Scoles, Urraca et al. 2011), and there is no robust experimental proof connecting increased expression of GABA receptors to seizure susceptibility.

Multiple mouse models have been generated to investigate Dup15q pathobiology, yet no model has recapitulated the seizure phenotypes observed in Dup15q individuals. Mice harboring a 6.3 Mb duplication of Chr 7 (syntenic to the 15q11.2-q13.1 region in humans) displayed social abnormalities and behavioral inflexibility when duplications were paternally inherited (Nakatani, Tamada et al. 2009), although Dup15q syndrome results primarily from maternally inherited duplications. Another mouse model duplicating only Ube3a recapitulated deficits in social interaction, social communication, and increased repetitive behavior but it is likely that the addition of a 3xFLAG tag to the C-terminus of Ube3a rendered the ubiquitin ligase activity of Ube3a-FLAG protein nonfunctional (Smith, Zhou et al. 2011). More recently, overexpression of Ube3a limited to neurons was not associated with seizures in mice but was linked to seizure-induced decreased sociability (Krishnan, Stoppel et al. 2017). Elevated levels of Ube3a in excitatory neurons in mice caused increased anxiety-like behaviors, cognitive impairments, and reduced seizure threshold to pharmacologically induced seizures with pentylenetetrazol, however spontaneous seizures have not been observed in these mice (Copping, Christian et al. 2017). In summary, mouse models of Dup15q which focus on

\footnotetext{
${ }^{2}$ Reprinted from final submission with permission. Hope, K.A., LeDoux, M.S., and Reiter, L.T. (2017). Glial overexpression of Dube3a causes seizures and synaptic impairments in Drosophila concomitant with down regulation of the $\mathrm{Na}^{+} / \mathrm{K}^{+}$pump ATP $\alpha$. Neurobiology of Disease, 108, 238-248. DOI: 10.1016/j.nbd.2017.09.003.
} 
neuronal overexpression of Ube3a have failed to recapitulate the spontaneous Dup $15 q$ seizure phenotype.

In contrast to neurons, glial cells biallelically express Ube3a (Yamasaki, Joh et al. 2003, Dindot, Antalffy et al. 2008, Judson, Sosa-Pagan et al. 2014, Grier, Carson et al. 2015). Analysis of post-mortem brain tissue from Dup15q individuals found elevated levels of $U B E 3 A$ transcript and protein (Scoles, Urraca et al. 2011). Even though glia play a key role in epileptogenesis through regulation of ion homeostasis (Chvatal and Sykova 2000, D'Ambrosio 2004, Devinsky, Vezzani et al. 2013), the functional consequence of increased glial UBE3A has been unexplored in Dup15q syndrome. Due to the absence of seizures in Dup15q mouse models, which have primarily focused on elevating Ube3a expression in neurons, we hypothesized that increased glial expression of UBE3A may contribute to the pathogenesis of epilepsy in Dup15q syndrome.

A related neurogenetic disorder, Angelman syndrome (AS), is also characterized by a high rate of epilepsy (Williams, Beaudet et al. 2006, Thibert, Conant et al. 2009). AS is caused by reciprocal deletions of the maternal 15q11.2-q13.1 region, methylation defects, or loss-of-function point mutations which result in non-functional UBE3A (LaSalle, Reiter et al. 2015). Due to the well-established link between UBE3A loss and seizures in AS, we predict that UBE3A may similarly underlie seizures in Dup15q.

The primary role of UBE3A, an E3 ubiquitin ligase, is to transfer ubiquitin moieties from an E2 ligase to substrate proteins, targeting them for degradation by the ubiquitin proteasome system (Finley 2009, Zheng and Shabek 2017). Several investigative teams, including our own, have long been searching for UBE3A substrates relevant to Dup15q and AS phenotypes. However, due to the transient nature of the interaction between an E3 ubiquitin ligase and its substrate proteins, only a handful of confirmed UBE3A substrates have emerged thus far (LaSalle, Reiter et al. 2015). As a result of the limited number of UBE3A substrates currently identified, the molecular mechanism of seizure production in Dup15q (and AS) individuals remains largely unknown.

Our laboratory previously used the genetically tractable model organism Drosophila melanogaster to identify Dube3a (the fly homolog of UBE3A) substrates in whole head fly extract following a transient elevation of Dube3a levels in all cell types (Jensen, Farook et al. 2013). We identified approximately 50 proteins whose expression was altered by increased $D u b e 3 a$ expression. One of these proteins was the sodium/potassium $\left(\mathrm{Na}^{+} / \mathrm{K}^{+}\right)$exchanger ATP $\alpha$, and we confirmed that ATP $\alpha$ is ubiquitinated in a Dube3a-dependent manner in vitro (Jensen, Farook et al. 2013). Prior work demonstrated that Drosophila with reduced levels of ATP $\alpha$ protein display a bangsensitive seizure phenotype (Schubiger, Feng et al. 1994, Sun, Xu et al. 2001, Palladino, Bower et al. 2003). Glial specific knockdown of the $\beta$ subunit of the $\mathrm{Na}^{+} / \mathrm{K}^{+}$pump $n r v 2$ also results in seizure-like behaviors ( $\mathrm{Ng}$, Sengupta et al. 2016), thereby supporting the idea that impaired glial-dependent regulation of extracellular ion concentration contributes to the pathogenesis of seizures. $\mathrm{Na}^{+} / \mathrm{K}^{+}$homeostasis is critically important for proper central nervous system function, while perturbation of $\mathrm{Na}^{+} / \mathrm{K}^{+}$balance has been 
identified as an underlying cause of epilepsy in humans (Somjen 2002, Scharfman 2007). Here, we follow up on the consequences of the interaction between Dube3a and ATP $\alpha$ in an attempt to understand the underlying mechanism of seizures in our novel fly model of Dup15q epilepsy.

\section{Methods}

\section{Fly Stocks}

All flies were maintained at $25^{\circ} \mathrm{C}$ on a 12 -hour light/dark cycle and raised on standard corn meal media (Bloomington Drosophila Stock Center). The following stocks were obtained from the Bloomington Drosophila Stock Center (Bloomington, IN): UASATP $\alpha$-RNAi (BDSC\# 32913), 10XUAS-IVS-myr::tdTomato (referred to as UAStdTomato, BDSC\#32222) and repo-GAL4 (BDSC\# 7415). The ATP $\alpha:$ GFP reporter line was generated by the FlyTRAP project (Morin, Daneman et al. 2001), and the UASATP $\alpha$ line was obtained from FlyORF (Bischof, Bjorklund et al. 2013). The following Dube3a and UBE3A lines were used in this study and described previously (Reiter, Seagroves et al. 2006): UAS-Dube $3 a$, UAS-Dube $3 a C / A$, and UAS-UBE3A. elav-GAL4 was provided by Dr. Hugo Bellen and UAS-GFP was provided by Dr. Cynthia Hughes.

\section{Quantitative Western Blot Analysis}

30-40 fly heads were removed and homogenized in RIPA buffer containing complete protease inhibitor cocktail (Roche). Samples were spun at 9,500 x g for 1 minute to remove debris and the supernatant containing protein was used for analysis. Samples were resolved by loading $20 \mu \mathrm{g}$ of protein into each lane of a $1.5 \mathrm{~mm}$ NuPAGE Bis-Tris 4-12\% gradient gel (Invitrogen) and transferred to Immobilon-FL PVDF membrane (Millipore). Membranes were blocked with 5\% bovine serum albumin, 3\% milk, and $0.1 \%$ Tween-20 in phosphate buffered saline (PBS). Primary antibodies used were $\alpha$-GAPDH (1:5000, IMGENEX Cat\# IMG-3073), $\alpha$-ATP $\alpha$ (1:5000, Developmental Studies Hybridoma Bank Cat\# A5-S) and $\alpha$-ELAV (1:200, Developmental Studies Hybridoma Bank Cat\# 7E8A10). $\alpha$-goat (LI-COR Cat\# 925-68074), $\alpha$-rat (LI-COR Cat\# 926-68076), and $\alpha$-mouse (LI-COR Cat\# 926-32212) infrared secondary antibodies were used at 1:7500. The blot was imaged using the Odyssey Infrared Imaging System (LiCor) and all lanes were normalized to a GAPDH loading control as the reference for calculating adjusted relative signal intensities.

\section{Immunohistochemistry and Image Acquisition}

Flies were briefly anesthetized with $\mathrm{CO} 2$, heads were removed and submerged in a dissecting dish containing PBS. Brains were dissected and fixed in $4 \%$ formaldehyde for 1 hour. Following 3 washes for 5 minutes in PBS, brains were blocked and 
permeabilized with $5 \%$ bovine serum albumin and $0.1 \%$ Triton X-100. Primary antibodies used in this study were mouse $\alpha$-repo (Developmental Studies Hybridoma Bank Cat\# 8D12, 1:100), mouse $\alpha$-brp (Developmental Studies Hybridoma Bank Cat\# NC82, 1:100), rat $\alpha$-Dube3a (Gift from Dr. Janice Fischer, 1:100), mouse $\alpha$-FasII (Developmental Studies Hybridoma Bank Cat\# 1D4, 1:100), and rabbit $\alpha$-GFP (Abcam Cat\# ab6556, 1:1000). Secondary antibodies used were AlexaFluor 594 goat $\alpha$-mouse secondary antibody (ThermoFisher Cat\# A11005, 1:500), AlexaFluor 488 goat $\alpha$-rat (ThermoFisher Cat\# A11006, 1:500), and AlexaFluor 488 goat $\alpha$-rabbit (ThermoFisher Cat\# A11008, 1:500). Slides were mounted with Vectashield mounting medium (Vector Labs Cat\# H-1200).

Images were captured on a Zeiss 710 confocal microscope (Zeiss) in the UTHSC Neuroscience Institute Imaging Core at 1024 x 1024 resolution with a $63 \mathrm{X}$ objective, numerical aperture 1.4. Z-sections were acquired at $1 \mu \mathrm{m}$ optical section thickness. Detector gain and offset were optimized to the control group and were not changed between brains once the configuration was set to allow for direct comparisons. Images were acquired with ZEN Software (Zeiss).

\section{Co-localization Analysis}

Co-localization analysis was performed using the ImageJ plugin Coloc 2 (Schneider, Rasband et al. 2012). A $50 \mu \mathrm{M}$ x $50 \mu \mathrm{M}$ section was selected from ATP $\alpha:$ :GFP brains stained with $\alpha$-GFP and $\alpha$-Dube3a antibodies for analysis.

\section{Seizure Susceptibility Assays}

For all behavioral tests, flies were not exposed to $\mathrm{CO}_{2}$ for at least two days prior to testing. BSA was performed as previously described (Benzer 1971, Ganetzky and Wu 1982, Stone, Burke et al. 2014). Flies were transferred via mouth pipette to empty standard fly vials and subjected to mechanical stress ("bang") by vortexing the vial on a standard laboratory vortexer (LabNet) at top speed for 10 seconds. At the end of the "bang", a timer was started and the duration of paralysis and uncontrolled movements was recorded. Flies were considered "recovered" when they righted themselves and were able to walk or groom.

For heat induced paralysis assays, flies were transferred to empty plastic fly vials and the vials were submerged in a $39^{\circ} \mathrm{C}$ water bath. The number of immobile flies was recorded in 30-s intervals and the assay continued for a total of $6 \mathrm{~min}$.

For photogenic-induced paralysis, flies were transferred to empty plastic fly vials and placed in a dark room for 30 min prior to testing. Vials were placed $5 \mathrm{~cm}$ from a strobe light (C4162 High Power Strobe, Chaney Electronics) and subjected to $10 \mathrm{~Hz}$ stimulation for $10 \mathrm{~s}$. Recovery time was recorded and was defined by the same parameters as for BSA. 


\section{Chemical Inhibition of ATPa Function}

Flies were fed ouabain (Sigma, 03125) using a previously described method (Sun, $\mathrm{Xu}$ et al. 2001). Briefly, three-day old flies were fed $10 \mathrm{mM}$ ouabain dissolved in water containing $5 \%$ sucrose for three hours by placing flies in an empty vial with containing a Kimwipe saturated with ouabain solution. Green food coloring (1\%) was included in the feeding solution to ensure flies consumed the ouabain solution, as indicated by their abdomens turning green. Following three hours of feeding, flies were tested for seizure susceptibility in the BSA.

\section{Glial Cell K $\mathrm{K}^{+}$Content Assay}

Stock solutions of $1 \mathrm{mM}$ Asante Potassium Green 2 (APG-2, TEFlabs, a fluorescent cell membrane permeable $\mathrm{K}+$ indicator) were prepared in DMSO. Three-day old repo $>$ tdTomato or repo $>$ Dube $3 a+t d$ Tomato flies were briefly anesthetized with $\mathrm{CO} 2$ and their heads were removed and submerged in room temperature Drosophila saline consisting of the following (in $\mathrm{mM}$ ): $128 \mathrm{NaCl}, 1.8 \mathrm{CaCl} 2,2 \mathrm{KCl}, 5 \mathrm{MgCl} 2,36$ sucrose, 5 HEPES, $\mathrm{pH}$ 7.2. Brains were dissected from the head case and incubated with $7.5 \mu \mathrm{M}$ APG-2 in Drosophila saline at room temperature with gentle agitation for 1 hour. Brains were washed $2 \mathrm{X}$ with Drosophila saline and wet mounted on a microscope slide with Drosophila saline.

Images were captured from the optic lobe on a Leica DM6000B microscope (Leica) using a 63X oil immersion lens using the N3 and L5 filters for tdTomato and APG-2 respectively. Exposure times were calibrated on repo $>$ tdTomato brains and microscope settings remained constant between repo $>$ tdTomato and repo $>$ Dube $3 a+t d$ Tomato groups to allow for direct comparison of fluorescence intensity. APG-2 fluorescence intensity was calculated offline using ImageJ (Schneider, Rasband et al. 2012). The APG-2 channel was converted to 8-bit greyscale, and tdTomato-positive glial cells were selected for analysis. Cells were selected using the "freeform" tool to outline the cell body in the APG-2 channel. Using the "measure" command, the mean fluorescence value for each cell was recorded. A total of 40 cells was analyzed per group from at least 3 images per group and 3 brains per group.

\section{Electroretinogram Analysis}

Electroretinograms (ERGs) were performed as previously described (Hope, LeDoux et al. 2016). Briefly, custom-made tungsten wire electrodes were gently inserted into the right eye (recording) and abdomen (ground) of immobilized flies. Fly eyes were stimulated using 1-sec light pulses from a 5-mm LED (RadioShack). Data was collected with a Model 1800 Microelectrode AC Amplifier (A-M Systems), digitized with a 
Micro3 1401 digitizer (Cambridge Electronic Design), and analyzed with Spike2 software (Cambridge Electronic Design).

\section{Electron Microscopy}

Fly heads were dissected and fixed in $2.5 \%$ glutaraldehyde solution overnight at $4^{\circ} \mathrm{C}$. Fixed heads were processed, resin embedded, and sectioned $(50 \mathrm{~nm})$ by the UTHSC Neuroscience Institute Core. A JEM-2000EXII microscope (JEOL USA, Inc.) at $60 \mathrm{kV}$ was used to capture transmission electron microscopy images at 10,000X.

\section{Statistical Analysis}

All statistical tests were performed using Prism version 6.0 (GraphPad). To test for statistical significance of two samples, Student's t-test was used. To determine statistical significance among multiple samples, we used a one-way ANOVA with Tukey's multiple comparisons. An alpha $(\alpha)$ of 0.05 was chosen for statistical significance.

\section{Results}

\section{Glia Endogenously Express both Dube3a and ATPa Proteins}

Dube $3 a$ is expressed broadly in neurons of the fly larval and adult brain (Wu, Bolduc et al. 2008). Our laboratory recently demonstrated that Dube3a is not subjected to the complex imprinting observed in mammals and is biallelically expressed in fly heads (Hope, LeDoux et al. 2016). UBE3A is known to be expressed solely from the maternal allele in mature mammalian neurons and biallelically expressed in mammalian glial cells (Yamasaki, Joh et al. 2003, Dindot, Antalffy et al. 2008, Judson, Sosa-Pagan et al. 2014, Grier, Carson et al. 2015). To determine if glial cells express Dube3a, we performed immunohistochemistry for the glial-specific transcription factor reversed polarity (Repo) and Dube3a in wildtype adult fly brains. We observed co-localization of Repo and Dube3a signals indicating that glial cells endogenously express Dube3a (Figure 4-1A).

Our laboratory previously found that the $\mathrm{Na}^{+} / \mathrm{K}^{+}$pump ATP $\alpha$ is ubiquitinated in a Dube3a-dependent manner using an in vitro ubiquitination assay (Jensen, Farook et al. 2013). For Dube3a to ubiquitinate ATP $\alpha$ in vivo, the two proteins must be present in the same cell. Therefore, we performed immunohistochemistry on brains from ATP $\alpha:$ GFP flies with antibodies against both GFP and Dube3a to determine if ATP $\alpha$ and Dube3a proteins co-localize in vivo. We observed a lattice-like staining pattern of ATP $\alpha:$ GFP in the optic lobes and relatively similar, yet more diffuse Dube3a staining patterns which overlapped with ATP $\alpha:$ GFP in several regions of the brain (Figure 4-1B, C). 

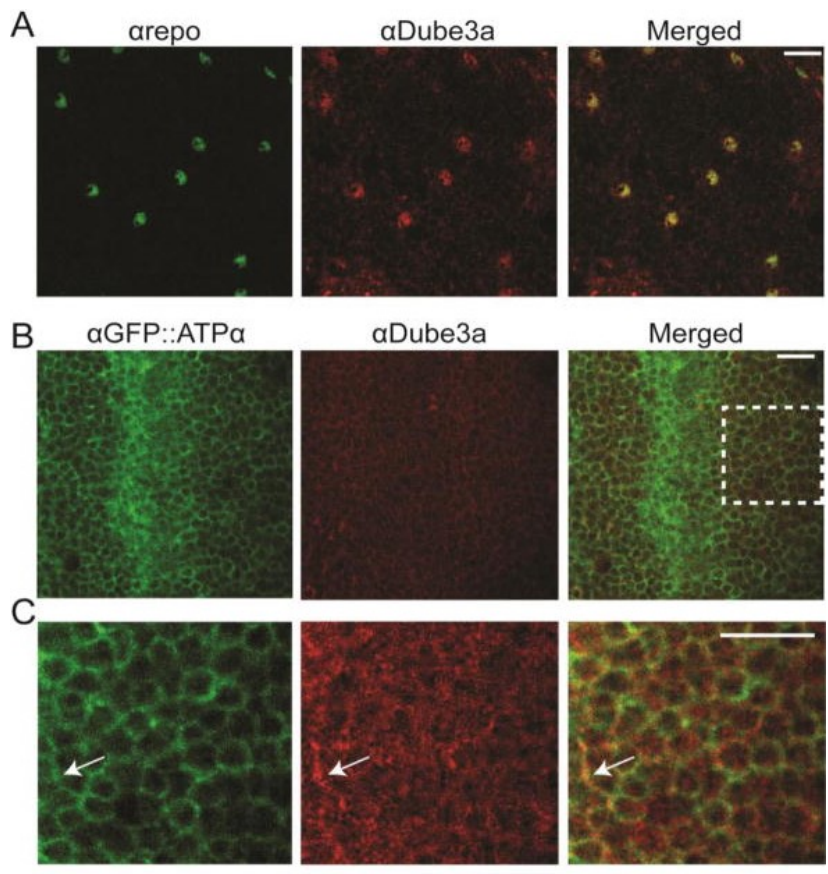

aDube3a

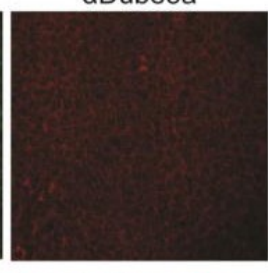

Merged
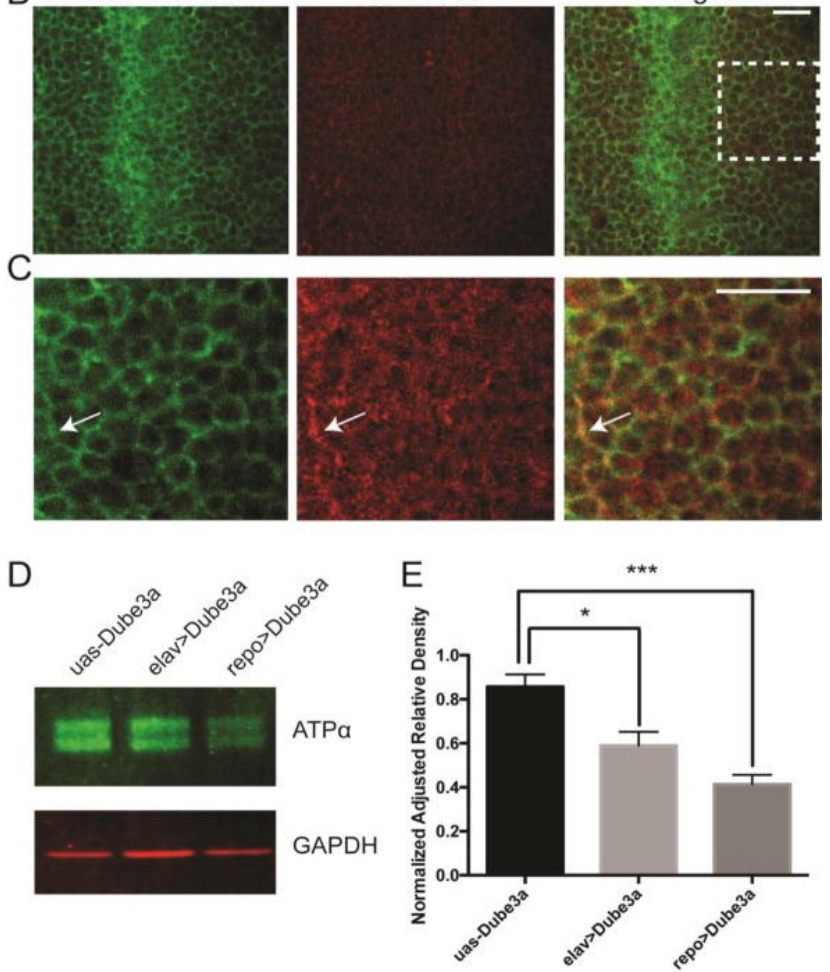

Figure 4-1. Glia endogenously express Dube3a and overexpression of Dube3a reduced ATP $\alpha$ protein levels in the brain.

A) Wild type adult fly brains stained for the glial specific transcription factor repo and E3 ubiquitin ligase Dube3a reveals a strong co-localization of the two signals, and Drosophila glia endogenously express Dube $3 a$. B) and C) Immunohistochemistry of adult wild type fly brains for ATP $\alpha$ and Dube3a revealed that Dube3a and ATP $\alpha$ colocalize. Cellular co-localization of the two proteins indicates the potential for Dube3a to directly ubiquitinate ATP $\alpha$ in vivo. Scale bar is 20 micrometers (Image $\mathbf{C}$ is a zoomed region indicated by the box in B). D) Western blot for ATP $\alpha$ levels from control (UASDube $3 a$ ), neuronal overexpression (elav $>$ Dube $3 a$ ), or glial overexpression $($ repo $>$ Dube3a) fly heads. E) Quantification of signals from D) indicate a significant decrease in ATP $\alpha$ levels in the brain. Note that Dube3a levels from repo $>$ Dube $3 a$ are reduced by greater than $50 \%$. A one-way ANOVA revealed a significant effect of genotype on ATP $\alpha$ levels $(\mathrm{p} \leq 0.001)$. Tukey's multiple comparisons test $(\alpha=0.05)$ indicated reduced levels of ATP $\alpha$ in elav $>$ Dube $3 a$ compared to UAS-Dube $3 a(\mathrm{p} \leq 0.05)$ and also in repo $>$ Dube $3 a$ compared to UAS-Dube $3 a(\mathrm{p} \leq 0.001) . \mathrm{n}=3$ for all samples, and data are presented as mean \pm SEM. 
Quantitative co-localization analysis indicated strong overlap of ATP $\alpha$ and Dube3a proteins $(r=0.78$, Figure 4-2). These data suggest that ATP $\alpha$ and Dube3a are present in at least some of the same cells in adult fly brain.

To determine whether Dube3a is able to regulate ATP $\alpha$ in vivo, we utilized the GAL4/UAS system (Brand and Perrimon 1993) to overexpress Dube3a in either neurons with elav-GAL4 or glia with repo-GAL4. We compared ATP $\alpha$ levels from fly heads via western blot from control UAS-Dube $3 a$ alone, elav $>D u b e 3 a$, and repo $>$ Dube $3 a$ animals (Figure 4-1D). There was a significant overall effect of genotype on ATP $\alpha$ levels (Figure 4-1E, One-way ANOVA, $P<0.001$ ). Post-hoc comparisons indicated that ATP $\alpha$ levels were significantly reduced in elav $>$ Dube $3 a$ compared to UAS-Dube $3 a$ alone $(P<$ $0.05)$ and in repo $>$ Dube $3 a$ compared to UAS-Dube $3 a$ alone $(P<0.001)$. These results are consistent with our previous studies indicating that Dube3a can regulate ATP $\alpha$ levels in the brain in a ubiquitin dependent manner.

\section{Overexpression of Dube3a in Glia Using repo-GAL4 Results in Seizure Susceptibility}

During our repo $>$ Dube $3 a$ experiments, we serendipitously observed a bangsensitive phenotype in repo $>$ Dube 3 a flies. To quantify this observation, we performed a series of seizure assays in both repo $>$ Dube $3 a$ and elav $>$ Dube $3 a$ animals. Utilizing the well-characterized bang sensitivity assay (BSA) (Benzer 1971, Ganetzky and Wu 1982, Stone, Burke et al. 2014), we subjected flies to mechanical stress and measured time to recovery. Control repo-GAL4 alone or neuronal overexpression elav $>$ Dube $3 a$ flies did not display a bang-sensitive phenotype, whereas glial Dube $3 a$ overexpression with repo $>$ Dube $3 a$ resulted in robust bang-induced paralysis (Figure 4-3A). We did not observe bang-sensitivity when we expressed catalytically-inactive ligase-dead Dube3a in glia using repo $>$ Dube $3 a C / A$ (Figure 4-3A), indicating that bang sensitivity requires the ubiquitin ligase activity of Dube3a. Additionally, we observed both heat-induced and photogenic paralysis in repo $>$ Dube $3 a$ animals (Figure 4-4A, B), indicating that seizures may be initiated by multiple modalities in repo $>$ Dube $3 a$ flies.

To evaluate the developmental time course of seizure susceptibility in repo $>$ Dube $3 a$ we measured bang-sensitivity at 0-2 days, 3-6 days, and 7-10 days posteclosion. We found that approximately $50 \%$ of repo $>$ Dube $3 a$ flies were bang-sensitive at 0-2 days post-eclosion and by 3-6 days all repo $>D u b e 3 a$ flies showed bang-sensitivity. By 7-10 days, no repo $>$ Dube $3 a$ flies recovered from the bang within the 2-min assay period (Figure 4-3B). These results indicate that seizures develop over time in adult flies overexpressing Dube $3 a$ in glia.

Because fly Dube3a and human UBE3A are greater than 70\% identical in the Cterminal HECT domain but display less conservation in the N-terminus (Reiter, Seagroves et al. 2006), we next wanted to determine if overexpression of human $U B E 3 A$ in fly glia also resulted in seizure susceptibility. In repo $>U B E 3 A$ flies, we observed a 

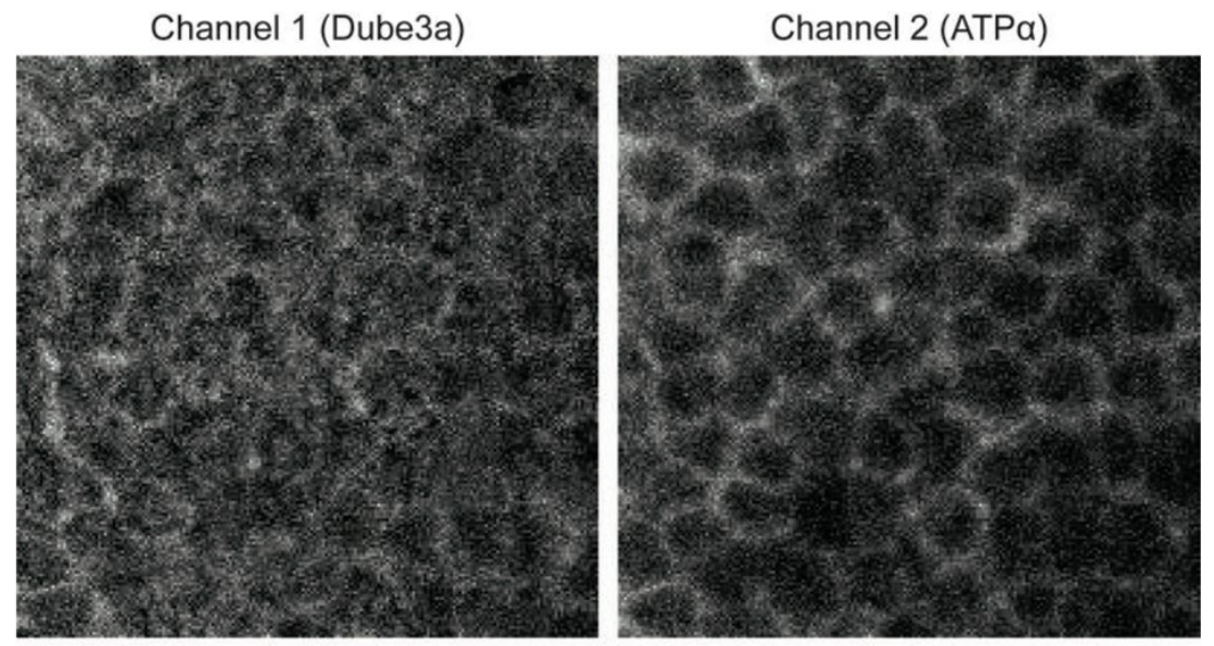

2D Intensity Histogram

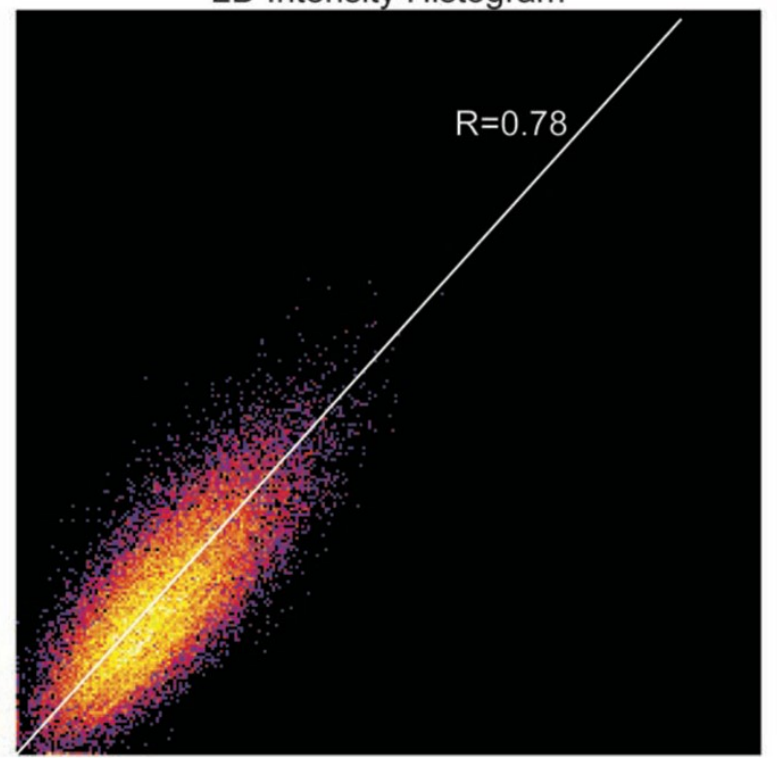

Figure 4-2. Co-localization analysis results from Dube3a and ATP $\alpha$ signals in the fly optic lobe.

Analysis generated from images in Figure 3-1C using the ImageJ plugin Coloc 2.

Channel 1 represents the Dube3a image channel converted to greyscale and channel 2 represents ATP $\alpha .2 \mathrm{D}$ intensity histogram represents the correlation of pixels across the two images. 
A

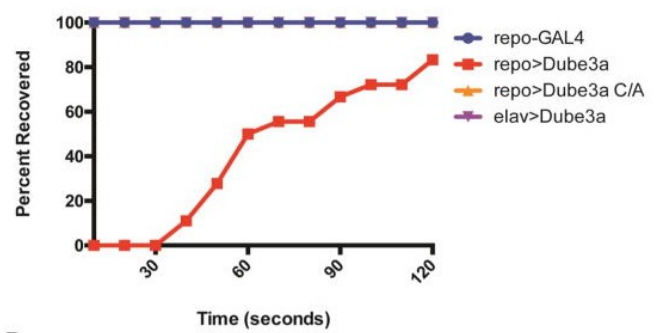

$$
\text { в }
$$

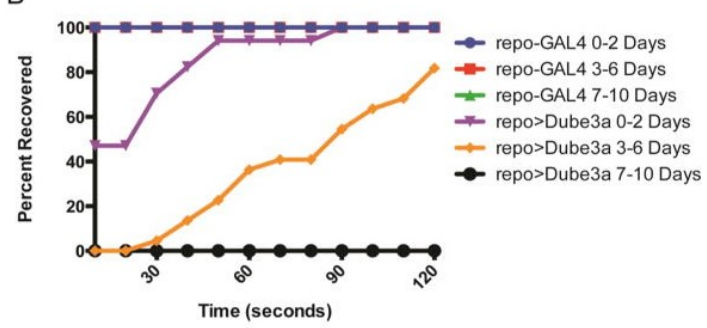

C

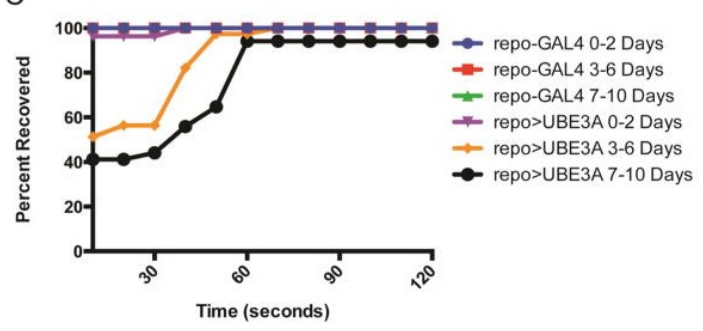

Figure 4-3. Elevated levels of Dube3a in glia result in seizure susceptibility in the bang-sensitivity seizure assay.

A) 3-5 day old flies from each genotype were tested in the BSA. Control (repo-GAL4, n = 15), overexpression of ligase dead Dube $3 a$ in glia $($ repo $>D u b e 3 a C / A, \mathrm{n}=10)$, neuronal overexpression of Dube $3 a$ (elav $>$ Dube $3 a, \mathrm{n}=12$ ), had no effect on seizure susceptibility ( $100 \%$ recovery at 10 seconds). Only overexpression of Dube $3 a$ in glia with repo $>$ Dube $3 a(\mathrm{n}=18)$ caused bang sensitivity $(80 \%$ recovery at 120 seconds $)$.

B) Seizures in repo $>$ Dube $3 a$ flies progressively worsened as flies aged. Control repoGAL4 flies did not display seizure activity at $0-2$ days $(\mathrm{n}=20), 3-6$ days $(\mathrm{n}=24)$, or 7 10 days $(\mathrm{n}=24)$ of age $(100 \%$ recovery at 10 seconds). Approximately $50 \%$ of repo $>$ Dube $3 a$ flies display seizures at $0-2$ days $(\mathrm{n}=17), 100 \%$ of repo $>$ Dube $3 a$ flies had seizures at 3-6 days $(\mathrm{n}=22)$ and recovered steadily over 2 minutes, and all repo $>$ Dube $3 a$ flies at $7-10$ days $(\mathrm{n}=4)$ had immediate seizure-like activity after BSA leading to paralysis and death (note the 3-6 day time point includes data from $2 \mathrm{~A}$ ). C) Overexpression of human $U B E 3 A$ in glia with repo $>U B E 3 A$ resulted in a similar, but less severe, seizure phenotype. At $0-2$ days $(\mathrm{n}=27)$, repo $>U B E 3 A$ flies displayed little seizure activity. However, by 3-6 $(\mathrm{n}=39)$ and $7-10(\mathrm{n}=34)$ days, repo $>U B E 3 A$ flies displayed seizures similar to that of 3-6 day old repo $>$ Dube $3 a$ flies. 


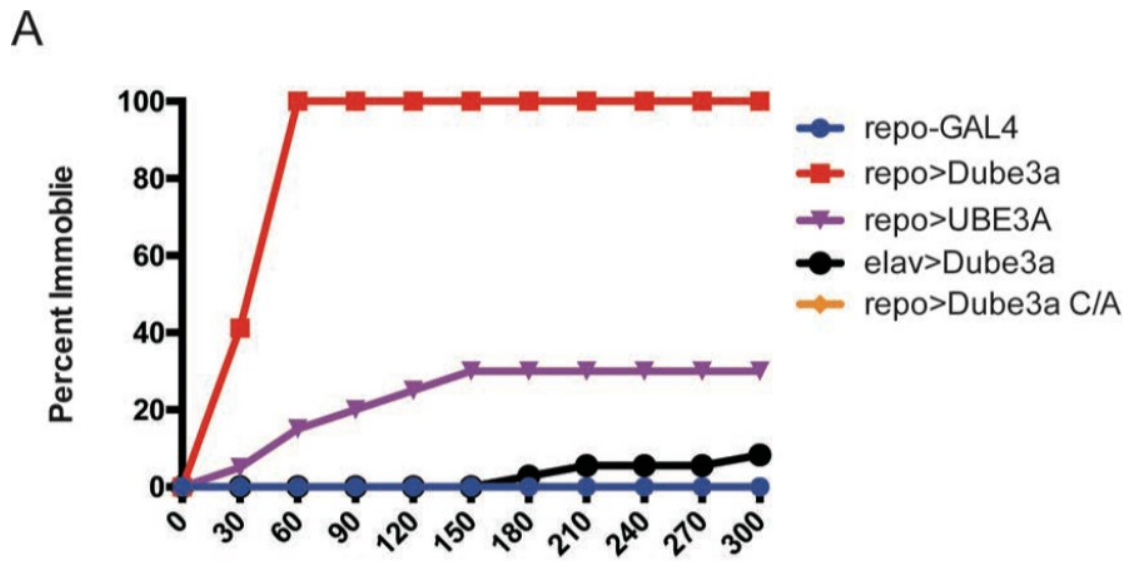

Time (seconds)

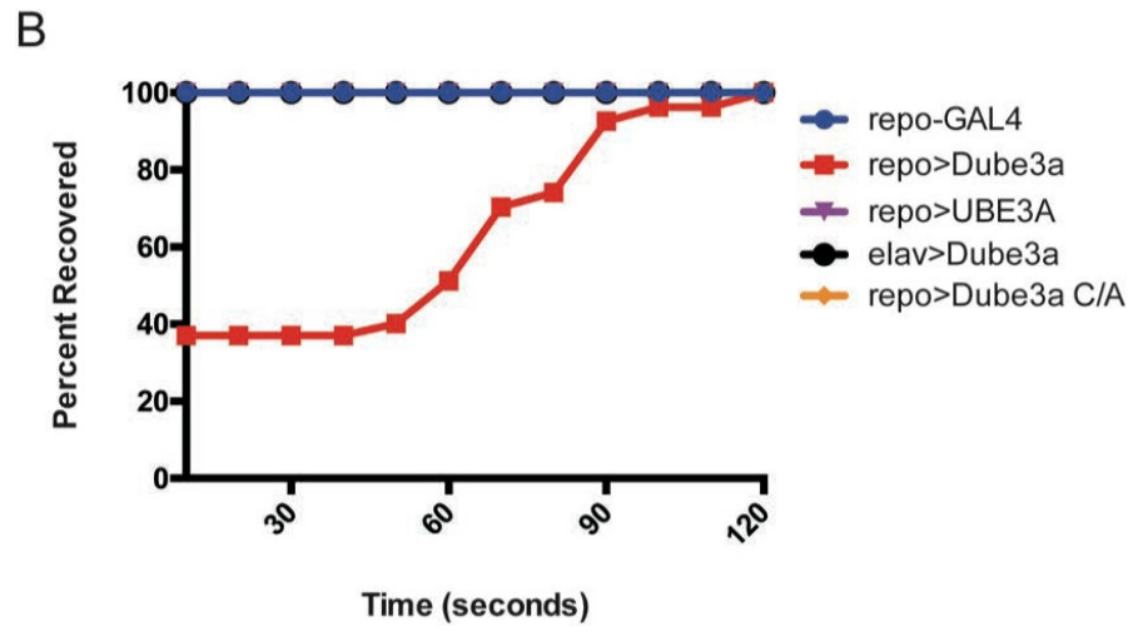

Figure 4-4. Seizures can be induced by heat or light in repo $>$ Dube3a animals. A) 3-5 day old flies were tested for heat-induced paralysis by elevating ambient temperature to $39^{\circ} \mathrm{C}$. This treatment had no effect on repo-GAL4 alone flies (blue line, $\mathrm{n}$ $=25$ ), however in repo $>$ Dube $3 a$ animals (red line, $\mathrm{n}=12$ ) all animals were paralyzed within 1 minute. We observed moderate heat-induced paralysis in repo $>U B E 3 A$ flies (purple line, $\mathrm{n}=20$ ), and a minimal effect in elav $>$ Dube $3 a$ animals (black line, $\mathrm{n}=36$ ). We observed no heat-induced paralysis when catalytically inactive Dube $3 a$ was expressed in glial cells with repo $>$ Dube $3 a C / A$ (orange line, $\mathrm{n}=25$ ) flies. B) Photogenic stimulation had no effect on repo-GAL4 alone flies (blue line, $\mathrm{n}=22$ ), elav $>$ Dube $3 a$ (black line, $\mathrm{n}=26$ ), or in ligase dead repo $>$ Dube $3 a C / A$ flies (orange line, $\mathrm{n}=23$ ).

Photogenic paralysis was observed in approximately $40 \%$ of repo $>$ Dube $3 a$ animals (red line, $\mathrm{n}=27$ ), however repo $>U B E 3 A$ animals were not susceptible to photogenic-induced paralysis (purple line, $\mathrm{n}=28$ ). 
similar but less severe bang-sensitivity that progressively worsened as flies aged just as repo $>$ Dube $3 a$ seizure severity progressively worsened in adult animals (Figure 4-3C). Moreover, repo $>U B E 3 A$ flies also demonstrated heat-induced paralysis, although heatsensitivity was less severe than in repo $>D u b e 3 a$ animals (Figure 4-4A). These experiments demonstrate that human UBE3A can emulate fly Dube3a in our Dup15q model implying shared substrate specificity between flies and humans for this E3 ubiquitin ligase.

Next, we capitalized on the temperature dependence of GAL4-driven transgene expression levels to investigate whether overexpression of Dube $3 a$ in early development or adulthood causes seizures (Figure 4-5A). Temperatures of $18^{\circ} \mathrm{C}$ reduce transgene expression, while $25^{\circ} \mathrm{C}$ induces transgene expression. Raising repo $>$ Dube $3 a$ flies at $18^{\circ} \mathrm{C}$ (repo $>$ Dube3a 18-18) from early embryonic stages through adulthood completely suppressed the seizure phenotype, as did raising repo $>$ Dube $3 a$ flies at $18^{\circ} \mathrm{C}$ during the embryo, larval, and pupal stages and then shifting to $25^{\circ} \mathrm{C}$ for five days before testing for seizures (Figure 4-5B, repo $>$ Dube $3 a$ 18-25). However, repo $>$ Dube $3 a$ flies raised at $25^{\circ} \mathrm{C}$ and overexpressing Dube $3 a$ in the embryo, larval, and pupal stages before reducing Dube $3 a$ expression in adulthood by shifting to $18^{\circ} \mathrm{C}$ on the day on the day of eclosion (repo $>$ Dube $3 a$ 25-18) displayed bang-sensitive seizures at 5 days of age (Figure 4-5B). These data suggest that overexpression of Dube $3 a$ in glia early in development is critical for the development of the seizure phenotype and initiates a seizure prone system that cannot be rescued by simply reducing Dube $3 a$ levels in adulthood.

\section{Genetic and Pharmacologic Manipulation of ATPa Modifies Seizure Susceptibility}

In order to establish that Dube3a mediated degradation of ATP $\alpha$ is the primary cause of seizures, we investigated ATP $\alpha$ protein stability and abundance in the brain under various conditions. We performed quantitative western blot analysis of ATP $\alpha$ levels in flies overexpressing human $U B E 3 A$ in glial cells to determine if ATP $\alpha$ is downregulated by human UBE3A protein, and on flies co-overexpressing ATP $\alpha$ on a repo $>$ Dube 3 a background to determine whether downregulation of ATP $\alpha$ could be genetically rescued (Figure 4-6A). There was a marked effect of genotype (Figure 4-6B, One-way ANOVA, $P<0.0001)$ but no significant differences between control repoGAL4 alone and ligase-dead overexpression repo $>$ Dube $3 a C / A$, indicating the downregulation of ATP $\alpha$ is dependent upon the ubiquitin ligase activity of Dube3a. Again, we observed significant downregulation of ATP $\alpha$ in repo $>$ Dube $3 a$ animals $(P<$ 0.01 ), similar to our earlier results (Figure 4-1D, E). No significant differences were observed in ATP $\alpha$ levels between control and ATP $\alpha$ overexpression animals $($ repo $>$ Dube $3 a+A T P \alpha)$ indicating that ATP $\alpha$ can be restored to near normal protein levels by simultaneously overexpressing ATP $\alpha$ in repo $>$ Dube $3 a$ flies (Figure 4-6A, B). Overexpression of human $U B E 3 A$ in repo $>U B E 3 A$ flies resulted in a similar downregulation of ATP $\alpha($ Figure 4-6A, B, $P<0.001)$ again suggesting conserved ubiquitin ligase substrates between fly Dube3a and human UBE3A. 
A

repo>Dube3a 18-18

\begin{tabular}{|c|c|}
\multicolumn{2}{c}{$18^{\circ} \mathrm{C}$} \\
\hline 10 days & 5 days \\
\hline Embryo/Larva/Pupa & Adult \\
\hline
\end{tabular}

repo>Dube3a 18-25

\begin{tabular}{|c|c|}
\hline $18^{\circ} \mathrm{C}$ & $25^{\circ} \mathrm{C}$ \\
\hline 10 days & 5 days \\
\hline Embryo/Larva/Pupa & Adult \\
\hline
\end{tabular}

repo>Dube3a 25-18

\begin{tabular}{|c|c|}
\hline $25^{\circ} \mathrm{C}$ & $18^{\circ} \mathrm{C}$ \\
\hline 10 days & 5 days \\
\hline Embryo/Larva/Pupa & Adult \\
\hline
\end{tabular}

B

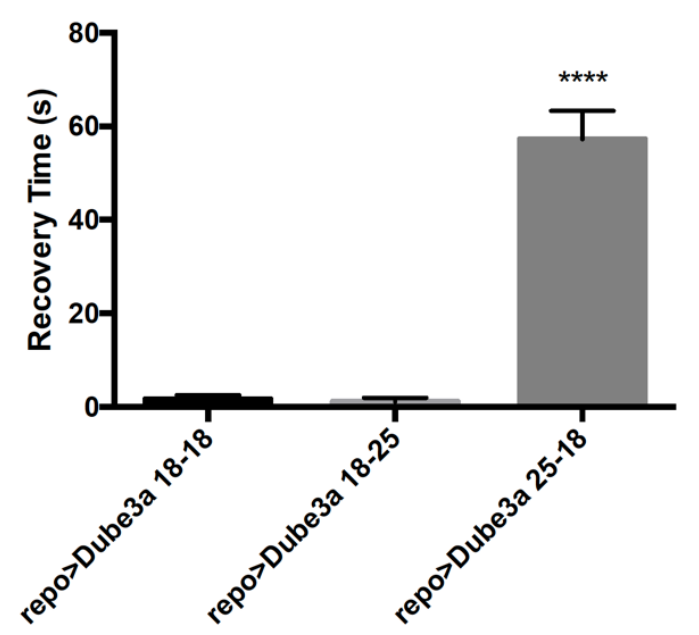

Figure 4-5. Glial Dube3a overexpression in early development, but not adulthood, causes bang-sensitive seizures.

A) Schematic for the temperature shifts to induce/repress Dube $3 a$ overexpression. Repo $>$ Dube 3 a 18-18 flies were raised at $18^{\circ} \mathrm{C}$ throughout development and adulthood. Repo $>$ Dube $3 a 18-25$ flies were raised at $18^{\circ} \mathrm{C}$ throughout development and shifted to $25 \mathrm{C}$ to induce Dube $3 a$ overexpression in adulthood. Repo $>$ Dube $3 a$ 25-18 flies were raised at $25^{\circ} \mathrm{C}$ (Dube3a overexpression during development) which was then turned off in adulthood by shifting to $18^{\circ} \mathrm{C}$ on the day of eclosion. All flies were tested for seizures at 5 days of age. B) Average recovery time in the bang sensitivity assay. Only repo $>$ Dube 3 a 25-18 displayed bang-sensitive seizures (One-way ANOVA, $\mathrm{p} \leq 0.0001, \mathrm{n}$ $\geq 8$ per genotype), indicating that overexpression of Dube $3 a$ in glia during development results in a seizure phenotype, but expression post eclosure alone does not cause seizures. 
A

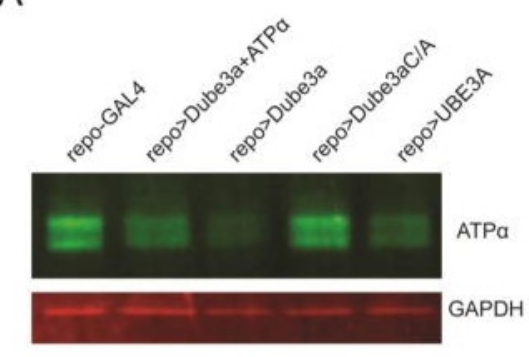

B

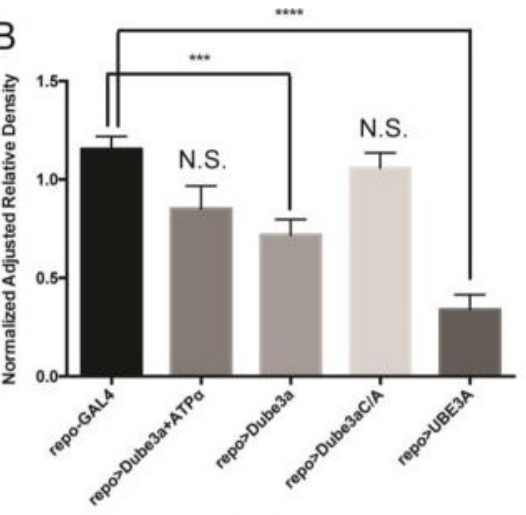

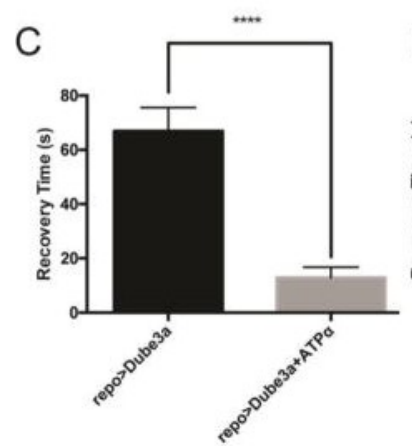
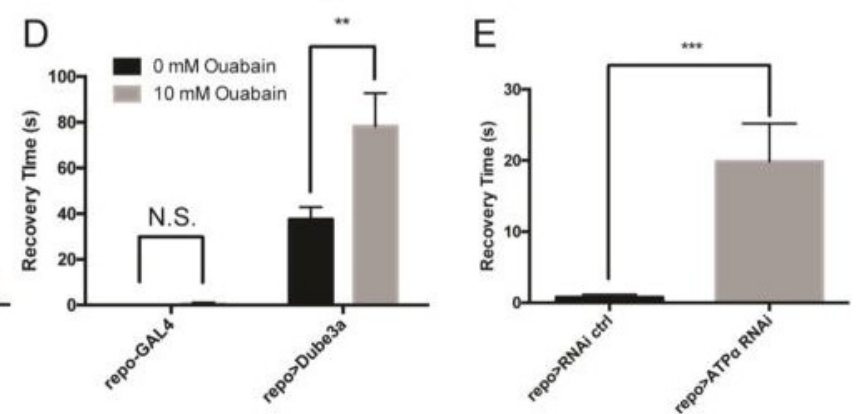

Figure 4-6. Genetic or pharmacologic manipulation of ATP $\alpha$ modulates seizure severity.

A) and B) We observed a significant effect of genotype on ATP $\alpha$ protein levels (OneWay ANOVA, $\mathrm{p} \leq 0.0001)$. ATP $\alpha$ protein levels are partially restored in repo $>$ Dube $3 a$ flies by concurrent overexpression of ATP $\alpha(p=0.30)$. We observed no reduction in ATP $\alpha$ when we overexpressed catalytically inactive Dube3a in glial cells $(p=0.09)$, but overexpression of human $U B E 3 A$ in glia similarly resulted in reduced levels of ATP $\alpha(\mathrm{p}$ $\leq 0.0001) . \mathrm{n} \geq 3$ for all blots. C) Overexpression of ATP $\alpha$ in repo $>$ Dube $3 a$ animals rescues seizure susceptibility ( $\mathrm{p} \leq 0.005, \mathrm{n} \geq 14$ all groups). D) Feeding repo $>$ Dube $3 a$ flies the $\mathrm{Na}^{+} / \mathrm{K}^{+}$pump inhibitor ouabain increases seizure severity $(\mathrm{p} \leq 0.01, \mathrm{n} \geq 9$ for all groups) likely due to the reduced levels of ATP $\alpha$. E) Glial specific knockdown of $A T P \alpha$ results in seizure susceptibility ( $\mathrm{p} \leq 0.005, \mathrm{n} \geq 14$ all groups). Data are presented as mean \pm SEM. 
We next performed BSA on repo $>$ Dube $3 a+A T P \alpha$ flies to determine if restoring ATP $\alpha$ levels specifically within glial cells of repo $>$ Dube $3 a$ animals was capable of rescuing seizure behavior. Compared to repo $>$ Dube $3 a$ flies, repo $>$ Dube $3 a+A T P \alpha$ animals had significantly reduced seizure severity as indicated by reduced seizure time on the BSA (Figure 4-6C, t-test, $P<0.005$ ). Therefore, reduced ATP $\alpha$ protein levels are necessary for seizure susceptibility in repo $>$ Dube $3 a$ flies.

The cardiotonic steroid ouabain is a known allosteric inhibitor of the $\mathrm{Na}^{+} / \mathrm{K}^{+}$ pump function of ATP $\alpha$. We predicted that repo $>$ Dube $3 a$ animals with reduced ATP $\alpha$ levels would be more susceptible to chemical ATP $\alpha$ inhibition and display longer recovery times on the BSA. We fed flies $10 \mathrm{mM}$ ouabain for 3 hours (a dose low enough to have no effect on control flies) and performed BSA (Figure 4-6D, t-test, $P \geq 0.05$ ). In repo $>$ Dube $3 a$ animals there was a significant increase in recovery time on the BSA (Figure 4-6D, t-test, $P<0.005$ ). These experiments confirm the connection between seizure susceptibility and ATP $\alpha$ function.

If Dube3a-dependent reduction in ATP $\alpha$ is responsible for seizures in repo $>$ Dube $3 a$ flies, then glial-specific knockdown of ATP $\alpha$ should also result in seizure susceptibility. Loss-of-function ATP $\alpha$ mutants are known to show seizure susceptibility, but these mutants have reduced ATP $\alpha$ throughout all tissues in the body (Schubiger, Feng et al. 1994). To determine if glial-specific knock down of $A T P \alpha$ is sufficient to cause seizures, we crossed repo-GAL4 flies to UAS- $A T P \alpha$-RNAi flies and performed the BSA. We observed significantly increased recovery times in repo $>A T P \alpha-R N A i$ flies compared to controls (Figure 4-6E, t-test, $P<0.005$ ), showing that glial-specific reduction of ATP $\alpha$ levels is sufficient to generate a seizure phenotype, albeit less severe than that found in repo $>$ Dube3a animals. This also implies that additional Dube3a glial substrates may contribute to the seizure phenotype.

\section{Glial Dube3a Overexpression Alters Neuronal Architecture}

We examined the mushroom body (MB) in adult flies to investigate the morphological effects of glial Dube $3 a$ overexpression. Immunohistochemistry was performed with an $\alpha$-FasII antibody in repo $>G F P$ and repo $>G F P+D u b e 3 a$ animals. The $\alpha$ lobe of the mushroom body normally extends dorsally in a single column and the $\gamma$ lobe displays FasII immunoreactivity as seen in control repo $>$ GFP brains (Figure 4-7A, top row). In the $\mathrm{MB}$ of repo $>G F P+D u b e 3 a$ flies we observed a loss of FasII immunereactivity in the $\gamma$ lobe and a misdirected portion of the $\alpha$ lobe (Figure 4-7A, bottom row, arrows). These results indicate that elevated levels of Dube3a in glia disrupt proper formation of the MB.

To further investigate neuronal architecture in glial Dube3a overexpression animals we stained adult fly brains with $\alpha$-brp antibody to visualize synaptic organization (Wagh, Rasse et al. 2006). The optic lobe (OL) of Drosophila displays a laminar structure with the outer medulla $(\mathrm{OMe})$ and inner medulla (IMe) separated by a column of glial 

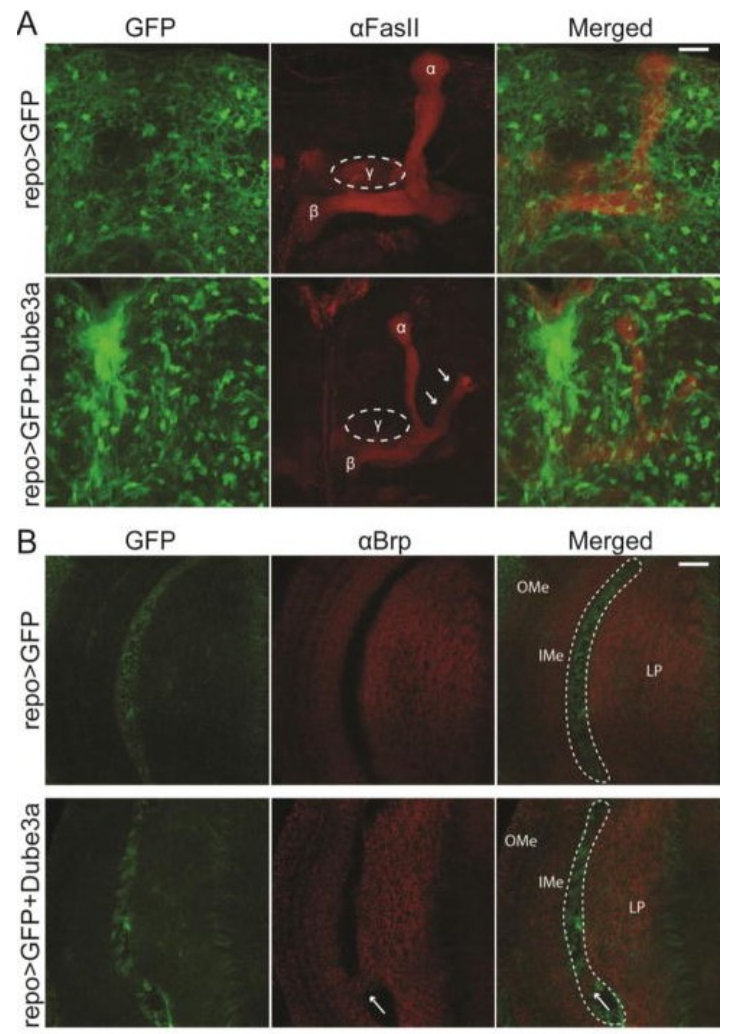

C
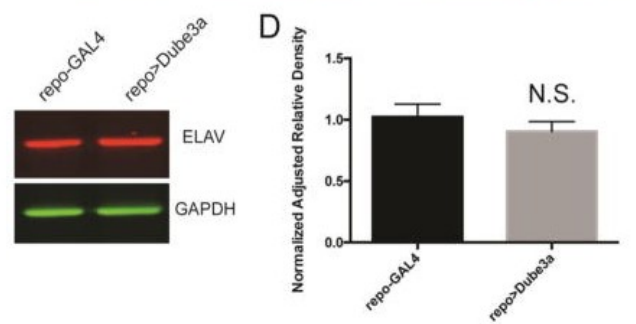

Figure 4-7. Overexpression of Dube3a in glia alters mushroom body and optic lobe morphology.

A) Adult repo $>G F P$ or repo $>G F P+D u b e 3 a$ fly brains stained with $\alpha$-FasII antibody to visualize the mushroom body (MB). In repo $>$ GFP animals (top row), $\alpha$-FasII marks $\alpha, \beta$, and $\gamma$ lobes, with the $\alpha$ lobe making a single dorsal projection. In repo $>G F P+D u b e 3 a$ (bottom row) flies we observed a loss of $\alpha$-FasII immunoreactivity in the $\gamma$ lobe (dashed circle) and a portion of the $\alpha$ lobe was misdirected (arrows). B) Adult repo $>$ GFP or repo $>G F P+D u b e 3 a$ fly brains stained with $\alpha$-Brp antibody to visualize neuronal organization in the optic lobe. In repo $>$ GFP animals (top row), $\alpha$-Brp reveals the laminar organization of the optic lobe consisting of the outer medulla (OMe), inner medulla (IMe), and lobula plate (LP), with the IMe and LP separated by a band of $\mathrm{GFP}^{+}$glial cells. In repo $>G F P+D u b e 3 a$ animals (bottom row) the laminar structure of the optic lobe is conserved, however $\mathrm{Brp}^{+}$projections are observed in the glial cell layer between the IMe and LP. Scale bar is 20 micrometers. C) and D) Western blot analysis of the neuronal marker elav in fly heads revealed no change in repo $>$ Dube $3 a(\mathrm{n}=3)$ vs. repoGAL4 $(\mathrm{n}=3)$ alone brains $(\mathrm{p}=0.42)$. Data are presented as mean $\pm \mathrm{SEM}$. 
cells from the lobular plate (LP) (Figure 4-7B, top row). Upon overexpression of Dube3a in glia we observed a similar laminar structures in the OL of repo $>G F P+D u b e 3 a$ brains, however $\alpha$-brp immunoreactivity was observed in the $\mathrm{GFP}^{+}$column of glial cells separating the IMe from the LP which was not observed in control flies (Figure 4-7B, bottom row, arrow). Again, these results indicate that overexpression of Dube $3 a$ in glial cells results in abnormal development of neuroanatomical structures.

Western blot analysis of the neuronal-specific transcription factor embryonic lethal abnormal vision (elav) was used to determine if reduced levels of ATP $\alpha$ in repo $>$ Dube $3 a$ flies was a consequence of reductions in the total number of fly neurons in the fly brains. We did not observe a difference in elav protein levels between repo-GAL4 and repo $>$ Dube $3 a$ animals (Figure 4-7C, $\mathbf{D}$, t-test, $\mathrm{P} \geq 0.05$ ), suggesting that neuronal loss is not the primary cause of seizures in repo $>$ Dube $3 a$ flies.

\section{Overexpression of Dube3a in Glia, but Not Neurons, Impairs Photoreceptor Neuron Function}

Seizures are thought to be primarily caused by excitatory/inhibitory imbalances in neurons. Functional changes in neuronal activity due to changes in Dube3a have been studied at the fly larval neuromuscular junction (Valdez, Scroggs et al. 2015), but synaptic transmission defects caused by overexpression of Dube $3 a$ have not been investigated in adult flies. Since seizures are caused by abnormal neuronal activity (Wu, Liu et al. 2015), we next wanted to directly measure neuronal function in the fly. Electroretinograms (ERGs) have long been used to measure synaptic activity and photoreceptor neuron function in the fly eye (Ugur, Chen et al. 2016). Using ERGs, we observed a significant effect of Dube $3 a$ or $U B E 3 A$ overexpression in both "on" transients (Figure 4-8A, B, One-way ANOVA, p < 0.0001) and "off" transients (Figure 4-8C, One-way ANOVA, $\mathrm{p}<0.0001$ ), as well as photoreceptor potentials (Figure 4-8A, D, One-way ANOVA, $p<0.0001)$. Tukey's multiple comparison test $(\alpha=0.05)$ indicated no effect of neuronal overexpression of Dube $3 a$ in elav $>$ Dube $3 a$ animals on any ERG component, though there was a significant difference between repo $>$ Dube $3 a$ and repo $>U B E 3 A$ compared to control repo-GAL4 alone flies in on/off transients and photoreceptor potentials. Again, no differences were observed between repo $>$ Dube $3 a C / A$ in any ERG component (Figure 4-8A through D), indicating the effects of Dube $3 a$ overexpression in glia are due to the ubiquitin ligase activity of Dube3a. Thus, Dube3a overexpression in glia, but not neurons, causes downstream synaptic defects in neurons in a cell non-autonomous manner.

To further investigate these ERG defects, we conducted an ultrastructural analysis of the fly eye using transmission electron microscopy. Ommatidial cross-sections of control repo-GAL4 and repo $>$ Dube $3 a$ animals revealed that both groups had similar stereotypic arrangements of photoreceptor neurons (Figure 4-9). In addition, secondary pigment glial cells are present and directly contact photoreceptor neurons in both repo- 
A
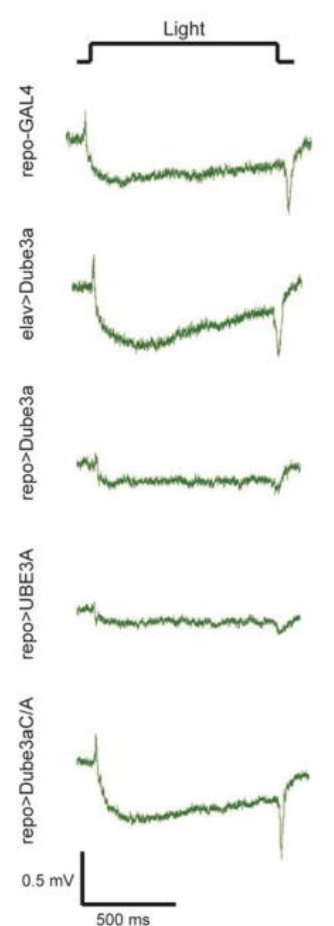

$\mathrm{E}$

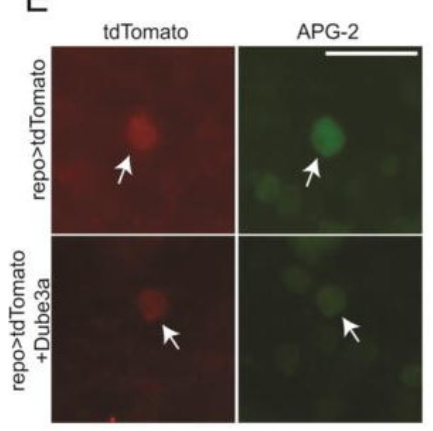

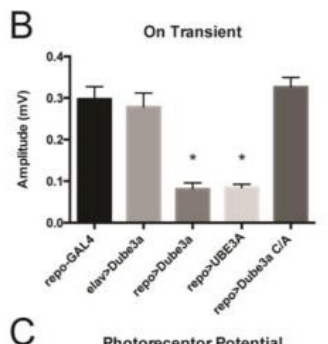


$\mathrm{F}$

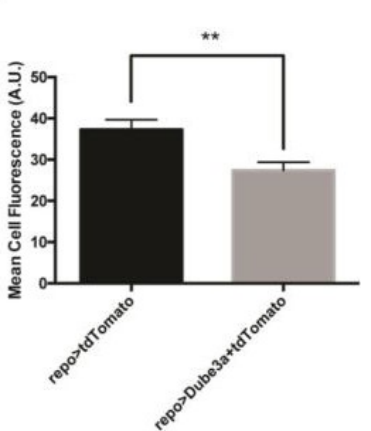

Figure 4-8. Overexpression of Dube3a in glia alters ERG signals and reduces internal $\mathrm{K}^{+}$levels in glial cells.

A) Control repo-GAL4 alone $(\mathrm{n}=10)$ ERGs display robust on/off transients and photoreceptor potentials. Overexpression of Dube $3 a$ in neurons with elav $>$ Dube $3 a,(\mathrm{n}=$ 8) or overexpression of ligase dead Dube $3 a$ in glia (repo $>$ Dube $3 a C / A, \mathrm{n}=8$ ) has no effect on the ERG signal. However, ERGs of glial overexpression of Dube3a (repo $>$ Dube $3 a, \mathrm{n}$ $=11$ ) or human $U B E 3 A$ (repo $>U B E 3 A, \mathrm{n}=8$ ) flies show reduced on/off transients and reduced photoreceptor potentials in 3-5 day old flies. B) Quantification of on transients. C) Quantification of photoreceptor potentials. D) Quantification of off transients. Data is presented as mean $\pm \mathrm{SEM}$. E) Using the fluorescent $\mathrm{K}^{+}$indicator Asante Potassium Green 2 (APG-2) and tdTomato to mark glial cells, we quantified internal potassium levels of control (repo $>$ tdTomato) and glial overexpression of Dube3a (repo $>$ tdTomato + Dube $3 a)$ glia. F) Quantification of the APG-2 fluorescence revealed a significant reduction in fluorescence signal ( $\mathrm{p} \leq 0.01)$, indicating reduced intracellular $\mathrm{K}^{+}$levels. Scale bar is 20 micrometers, and $n=40$ cells from 3 flies per group. Data are presented as mean $\pm \mathrm{SEM}$. 

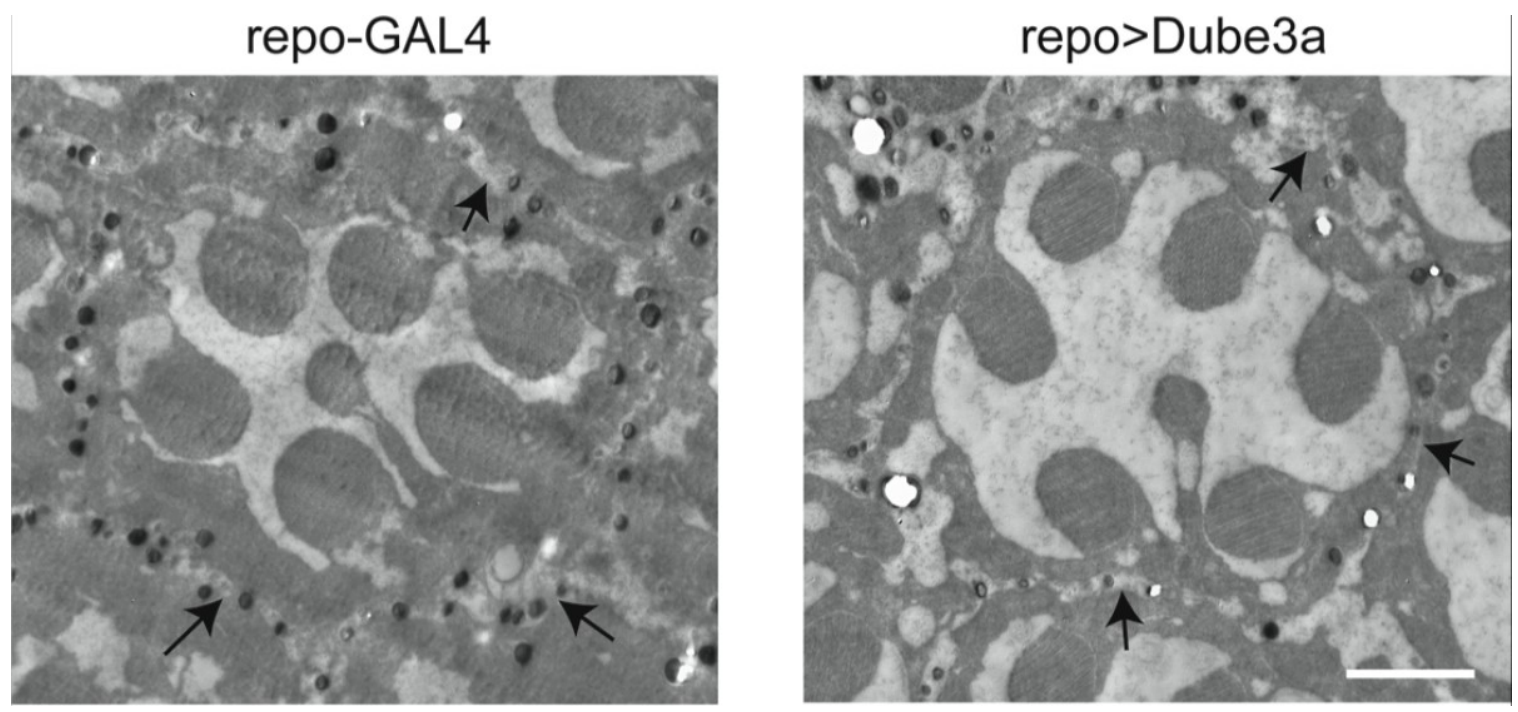

Figure 4-9. Transmission electron microscopy of control (repo-GAL4) or glial Dube $3 a$ overexpression (repo>Dube $3 a$ ) ommatidia of 5 day old flies.

Pigment cells (glia) surround and contact photoreceptor neurons in both repo-GAL4 and repo $>$ Dube $3 a$ (arrows), indicating ERG defects are not due to a failure of glial cells to contact neurons. Scale bar is 2 micrometers. 
GAL4 and repo $>$ Dube 3 a flies (Figure 4-9, arrows), indicating abnormal ERGs do not arise from gross photoreceptor defects or a lack of physical interaction between supporting glial cells and photoreceptor neurons.

\section{Glial-specific Overexpression of Dube3a Causes Reduced Intercellular $\mathrm{K}^{+}$in Glial Cells}

The function of ATP $\alpha$ is to pump $\mathrm{K}^{+}$into the cell while pumping $\mathrm{Na}^{+}$into the extracellular space in an ATP-dependent manner. Since repo $>$ Dube $3 a$ animals have reduced levels of ATP $\alpha$, we predicted that glial cells in the fly brain would have reduced intracellular $\mathrm{K}^{+}$concentrations. We dissected whole brains from repo $>$tdTomato or repo $>$ tdTomato + Dube $3 a$ animals to visualize glia and incubated them with the cellpermeant fluorescent $\mathrm{K}^{+}$indicator Asante Potassium Green 2 AM (APG-2). We then quantified the fluorescence of APG-2 in tdTomato-positive cells to determine the relative concentration of $\mathrm{K}^{+}$in repo $>$tdTomato and repo $>$tdTomato + Dube $3 a$ glial cells. We observed a significant reduction in APG-2 fluorescence in repo $>$ tdTomato + Dube $3 a$ compared to control repo $>$ tdTomato glial cells (Figure 4-8E, F, t-test, $\mathrm{P}<0.01$ ). These data indicate that elevated Dube3a levels within glia reduce internal $\mathrm{K}^{+}$, consistent with the hypothesis that Dube3a downregulates ATP $\alpha$ levels causing a disruption of $\mathrm{Na}^{+} / \mathrm{K}^{+}$ homeostasis.

\section{Discussion}

To date, animal models of Dup15q focused on neuronal overexpression of $U B E 3 A$ have failed to recapitulate the seizure phenotypes observed in Dup15q. Our fly model of Dup15q with elevated levels of Dube3a in glia, but not neurons, displays a robust seizure phenotype, highlighting the previously unappreciated role that glia play in Dup $15 \mathrm{q}$ pathophysiology. Our lab previously demonstrated that Dube3a is biallelically expressed in the Drosophila brain (Hope, LeDoux et al. 2016), and here we demonstrate that Drosophila glia endogenously express $D u b e 3 a$ in addition to expression in neurons. Studies in mammalian systems have indicated biallelic expression of Ube $3 a / U B E 3 A$ in glial cells (Yamasaki, Joh et al. 2003, Dindot, Antalffy et al. 2008, Judson, Sosa-Pagan et al. 2014, Grier, Carson et al. 2015), yet until now the functional consequence of overexpression of Ube $3 a / D u b e 3 a$ in glial cells has been largely unexplored. Increased dosage of the E3 ubiquitin ligase UBE3A is thought to be the underlying cause of at least the autistic features of Dup15q syndrome (LaSalle, Reiter et al. 2015), and evidence presented here suggests that elevated UBE3A contributes to epilepsy in Dup15q as well.

Drosophila harboring loss-of-function mutations in ATP $\alpha$ have previously shown a bang-sensitive phenotype (Schubiger, Feng et al. 1994, Sun, Xu et al. 2001, Palladino, Bower et al. 2003), and bang-sensitivity is a simple yet robust measure of seizure-like activity in flies (Benzer 1971, Ganetzky and Wu 1982, Stone, Burke et al. 2014). Here we utilized the GAL4/UAS system (Duffy 2002) to selectively knockdown ATP $\alpha$ levels in glial cells. This approach revealed the importance of $\mathrm{Na}^{+} / \mathrm{K}^{+}$regulation within glial cells 
on proper central nervous system function, as glial-specific knockdown of ATP $\alpha$ generated a bang-sensitive seizure phenotype. Glial dysfunction is well-recognized as a major contributor to epilepsy (Wetherington, Serrano et al. 2008, Devinsky, Vezzani et al. 2013), and the data presented herein suggests that glial dysfunction contributes to the pathogenesis of epilepsy in Dup15q.

One role of glial cells is to maintain ion homeostasis in the central nervous system. Normally, $\mathrm{K}^{+}$is pumped into glial cells via ATP $\alpha$ to maintain relatively low concentrations of $\mathrm{K}^{+}$in the extracellular space (Figure 4-10A). When Dube3a (or UBE3A) is elevated in glia, ATP $\alpha$ levels are reduced, thus reducing the amount of intracellular $\mathrm{K}^{+}$within glial cells and increasing the concentration of $\mathrm{K}^{+}$in the extracellular space (Figure 4-10B). We propose that elevated extracellular $\mathrm{K}^{+}$leads to the neuronal dysfunction and seizure behavior observed in repo $>$ Dube $3 a$ flies. $\mathrm{Na}^{+} / \mathrm{K}^{+}$ dysregulation has emerged as a potential cause of epilepsy as elevated levels of extracellular $\mathrm{K}^{+}$lead to more positive neuronal membrane potentials, tipping neuronal networks into hyper-excitable states and precipitating seizure activity (Scharfman 2007, Seifert and Steinhauser 2013). Our data indicate both reduced levels of the $\mathrm{Na}^{+} / \mathrm{K}^{+}$pump and reduced intracellular $\mathrm{K}^{+}$levels in glial cells of repo $>$Dube $3 a$ animals which is likely the underlying mechanism for seizure susceptibility in our fly model, and potentially Dup15q syndrome where UBE3A levels are known to be elevated (Scoles, Urraca et al. 2011).

The glial specific transcription factor reversed polarity (repo) was initially identified in a screen for mutants affecting the physiology of the ERG signal, and repo mutants displayed an ERG with a positive polarity rather than the typical negative polarity upon photoreceptor stimulation (Xiong, Okano et al. 1994). Pigment glial cells of the eye ensheath photoreceptor neuron cell bodies while epithelial glia surround axon terminals (Edwards and Meinertzhagen 2010). These glial cells in the Drosophila eye strongly express $A T P \alpha$ (Gorska-Andrzejak 2013). It is not surprising then that overexpression of Dube 3 a specifically within glial cells using the repo-GAL4 driver line causes defects in the ERG signal, potentially through the downregulation of ATP $\alpha$.

Identifying disease-relevant substrates of UBE3A has remained elusive. We previously identified ATP $\alpha$ as a ubiquitin ligase substrate of Dube3a (Jensen, Farook et al. 2013), and here we demonstrate that overexpression of Dube3a in both glia and neurons results in reduced levels of $\mathrm{ATP} \alpha$, confirming our previous work. Glial specific knockdown of ATP $\alpha$ recapitulates a portion of the seizure phenotype observed in repo $>$ Dube $3 a$ animals, indicating that reduced levels of ATP $\alpha$ in glia alone is sufficient to generate seizures. Even though we did not detect a significant difference in ATP $\alpha$ protein levels between repo-GAL4 and repo $>$ Dube $3 a+A T P \alpha$, we did not fully rescue the seizure phenotype in repo $>$ Dube $3 a+A T P \alpha$ compared to repo $>D u b e 3 a$ flies. It is possible that slight decreases in ATP $\alpha$ protein levels undetectable by western blot may still result in seizure susceptibility, or that other unidentified proteins and pathways regulated by Dube3a contribute to seizure. These other targets may contribute substantially to the 
A

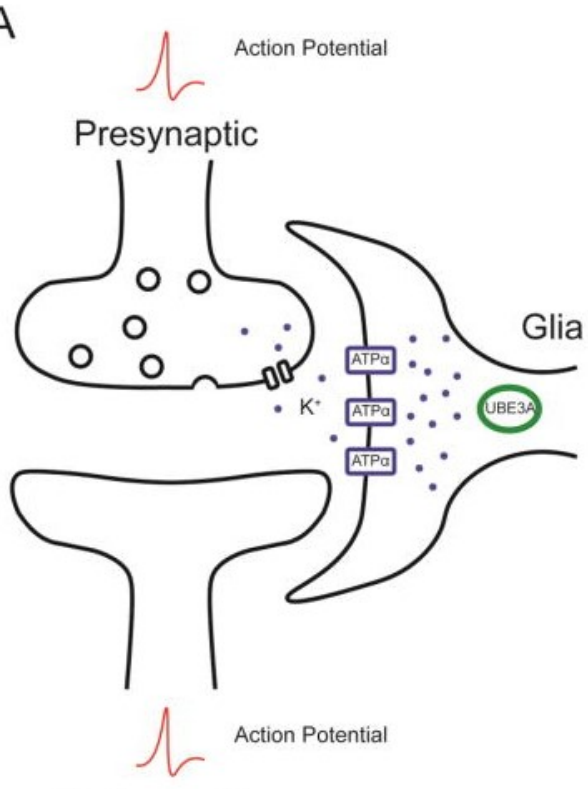

Postsynaptic
B
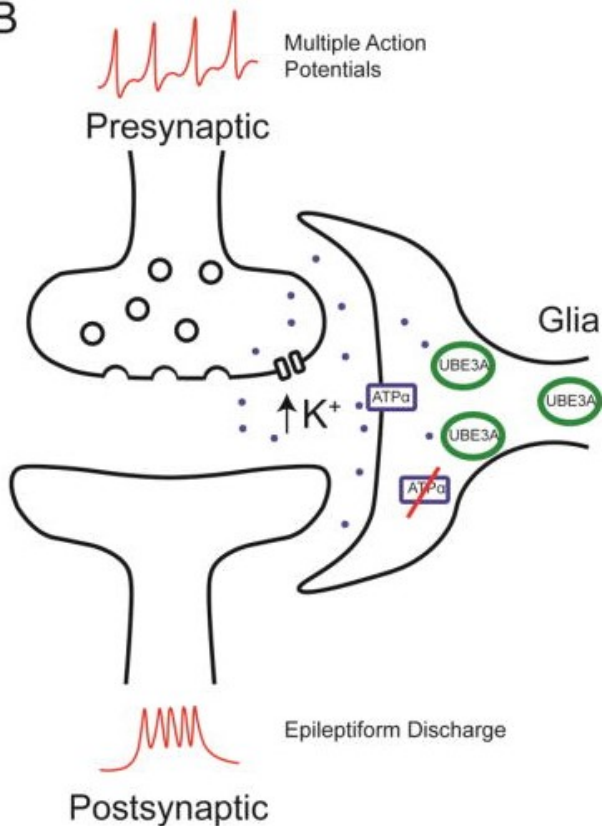

Figure 4-10. Proposed mechanism of seizure in repo $>$ Dube3a animals.

A) In control flies, relatively low levels of Dube3a are present in glia, while high levels are $\mathrm{ATP} \alpha$ are present in the glial membrane. As $\mathrm{K}^{+}$effluxes from neurons, ATP $\alpha$ removes $\mathrm{K}^{+}$from the synaptic cleft, restoring $\mathrm{K}^{+}$gradients required for proper neuronal function. B) In repo $>$ Dube $3 a$ animals, elevated levels of Dube3a in glia reduce ATP $\alpha$ levels in the membrane. As $\mathrm{K}^{+}$effluxes from neurons it is not cleared from the extracellular space, leading to hyperpolarized neurons and increasing neuronal activity, resulting in epileptiform activity and seizures. 
variability in seizure severity in Dup15q syndrome (Battaglia 2008, Hogart, Leung et al. 2009). Taken together, reduced levels of ATP $\alpha$ due to elevated levels of Dube3a seems to be driving a significant portion of the seizure phenotype observed in repo $>$ Dube $3 a$ flies.

Human UBE3A and Drosophila Dube3a are approximately $70 \%$ identical at the $\mathrm{C}$-terminus. The two proteins are less conserved in the N-terminus which is believed to determine substrate protein binding (Cooper, Hudson et al. 2004, Scheffner and Kumar 2014). We observed reductions in ATP $\alpha$ levels in both repo $>D u b e 3 a$ and repo $>U B E 3 A$ flies, suggesting that ATP $\alpha$ is a substrate of both fly Dube3a and human UBE3A. The ability of UBE3A to downregulate ATP $\alpha$ is similar to or possibly exceeds the ability of Dube3a to downregulate $\mathrm{ATP} \alpha$, yet we observed less severe seizure susceptibility in repo $>U B E 3 A$ compared to repo $>$ Dube $3 a$ flies. As stated above, this discrepancy in seizure severity may result from other substrate proteins or pathways that are not conserved between Dube3a and UBE3A which contribute to seizure susceptibility and variability. Indeed, we observed a partial rescue of seizure susceptibility when ATP $\alpha$ levels were restored to near normal levels in repo $>$ Dube $3 a$ flies, indicating that downregulation of ATP $\alpha$ plays a large role in seizure susceptibility in repo $>$ Dube $3 a$ flies. However, seizures were not fully rescued by restoring ATP $\alpha$ levels, suggesting that mechanisms other than the downregulation of ATP $\alpha$, which may not be shared by Dube3a and UBE3A, contribute to seizure susceptibility. This data is further supported by our observations that glial specific knockdown of ATP $\alpha$ generates a seizure phenotype similar in severity to repo $>U B E 3 A$ flies, both of which are less severe than repo $>$ Dube $3 a$ flies. It is reasonable to propose, therefore, that mechanisms contributing to seizure other than downregulation of ATP $\alpha$ remain to be identified.

Mammals have 3 homologs to ATP : ATP1A1, ATP1A2, and ATP1A3. While $A T P 1 A 1$ is ubiquitously expressed and $A T P 1 A 3$ is primarily found in neurons, ATP1A2 is predominantly expressed in astrocytes (McGrail, Phillips et al. 1991). ATP1A2 mutations cause familial hemiplegic migraine type 2 with epileptic seizures (OMIM 602481) (De Fusco, Marconi et al. 2003, Vanmolkot, Kors et al. 2003, Jurkat-Rott, Freilinger et al. 2004, Spadaro, Ursu et al. 2004, Swoboda, Kanavakis et al. 2004, Deprez, Weckhuysen et al. 2008, Gallanti, Tonelli et al. 2008), providing further evidence that impaired $\mathrm{Na}^{+} / \mathrm{K}^{+}$ pump function in glial cells causes epilepsy. It remains to be determined whether $\mathrm{Na}^{+} / \mathrm{K}^{+}$ pump levels are affected in the brains of individuals with Dup15q.

In repo $>$ Dube $3 a$ flies we observed abnormal neuroanatomical structures in both the MB and OL. Previous reports have indicated abnormal MB morphology in homozygous Dube3a loss-of-function flies (Chakraborty, Paul et al. 2015). Here we demonstrate that glial overexpression of Dube3a causes MB defects in a cell nonautonomous manner. Hippocampal heterotopias and malformations have been observed in Dup15q individuals antemortem via MRI and post-mortem (Wegiel, Schanen et al. 2012, Boronat, Mehan et al. 2015). Our data suggests that elevated glial expression of $U B E 3 A$ may contribute to the development of hippocampal heterotopias and malformations observed in Dup15q syndrome. Glia play a crucial role in the development and organization of neural circuits (Stiles and Jernigan 2010), such that altering glial function could profoundly impact neuronal pathfinding, altering the structure of the 
central nervous system and affecting neuro-behavioral phenotypes such as autism or intellectual disability in Dup15q individuals.

This is the first model of Dup15q syndrome that recapitulates the seizure phenotype observed in Dup15q individuals as currently available mouse models do not develop epilepsy (Nakatani, Tamada et al. 2009, Smith, Zhou et al. 2011, Krishnan, Stoppel et al. 2017). Dup15q mouse models have focused on overexpression of Ube3a in neurons, since $U b e 3 a$ is only imprinted in neurons while glia, which biallelically express Ube $3 a$, have been largely ignored. During early experiments we serendipitously observed a bang-sensitive seizure phenotype in repo $>$ Dube $3 a$ animals simply by placing a fly vial on the bench top or opening the incubator door, indicating a relatively severe seizure phenotype. Individuals with Dup15q syndrome often manifest a pharmacologically resistant form of epilepsy that progressively worsens over time, exhibiting infantile spasms which progress to Lennox-Gastaut type syndrome (Finucane, Lusk et al. 2016). Repo $>$ Dube $3 a$ animals displayed a relatively mild seizure phenotype at $0-2$ days of age but by 7-10 days displayed a severe seizure behavior, similar to the progression of seizures in Dup15q syndrome. These observations imply that seizures are not fixed but have a developmental trajectory, suggesting that early intervention strategies may be capable of preventing or reversing seizures.

The data presented here indicate that glial cells, not neurons, are the key cell type responsible for seizure development in Dup15q syndrome through dysregulation of ATP $\alpha$-mediated $\mathrm{K}^{+}$homeostasis. Recognition that glial cells contribute to the pathophysiology and clinical manifestations of Dup15q syndrome represents a paradigm shift in the study of Dup15q syndrome, UBE3A, and autism in general. 


\section{CHAPTER 5. TRANSCRIPTOMIC AND PROTEOMIC PROFILING OF GLIAL VERSUS NEURONAL DUBE3A OVEREXPRESSION REVEALS COMMON MOLECULAR CHANGES IN GLIOPATHIC EPILEPSIES}

\section{Introduction}

Epilepsy affects approximately $1 \%$ of humans worldwide in their lifetime (Fiest, Sauro et al. 2017). Despite advances in epilepsy treatment, uncontrolled epilepsy remains common and approximately $30-50 \%$ of people with epilepsy ultimately develop treatment resistant seizures (Kwan and Brodie 2000, Golyala and Kwan 2017, LaPenna and Tormoehlen 2017). Investigating the mechanism by which treatment resistant seizures occur and identifying molecular commonalities among different genetic causes of seizures, can help fill the gap in our knowledge about why epilepsy is refractory to many current treatments.

One genetic disorder with a high rate of treatment resistant epilepsy that may serve as an entry point into investigating treatment resistant seizures is Duplication 15q syndrome, or Dup15q (Finucane, Lusk et al. 2016). Dup15q results from maternally derived duplications of chromosome 15q11.2-q13.1 and individuals with Dup15q harbor 1 to 6 extra copies of this region. A retrospective survey on seizure in Dup15q indicated that seizure severity was positively correlated with $15 \mathrm{q} 11.2-\mathrm{q} 13.1$ copy number as $25 \%$ of interstitial duplications ( 1 extra copy), 64\% of isodicentric duplications ( 2 extra copies), and $100 \%$ of complex duplications (up to 6 extra copies) presented with seizures. As low as $25 \%$ of individuals with isodicentric Dup $15 \mathrm{q}$ had a $>90 \%$ reduction in seizures after the first medication, and seizures were refractory in roughly $75 \%$ of epileptic individuals (Conant, Finucane et al. 2014).

The majority of Dup $15 \mathrm{q}$ research has focused on neuronal overexpression of the E3 ubiquitin ligase $U B E 3 A$. Due to the complex imprinted regulation in neurons at the 15q11.2-q13.1 locus, $U B E 3 A$ is only expressed from the maternal allele in mature mammalian neurons (LaSalle, Reiter et al. 2015). Dup15q characteristics other than seizures have been successfully recapitulated in mouse models, such as social abnormalities and behavioral inflexibility (Nakatani, Tamada et al. 2009), social interaction deficits (Smith, Zhou et al. 2011), and seizure-induced decreased sociability (Krishnan, Stoppel et al. 2017). However, modeling seizure susceptibility itself has been difficult to do in rodent models. A recent mouse model restricting Ube3a overexpression to excitatory neurons caused slightly reduced threshold to pharmacologically induced seizures, yet spontaneous seizure behavior was not described (Copping, Christian et al. 2017).

To develop a seizure model of Dup15q, we turned to Drosophila melanogaster and the powerful GAL4/UAS system (Brand and Perrimon 1993, Duffy 2002). This new Dup15q model in flies recapitulates the seizure phenotype when Dube $a$ (the fly UBE3A homolog) or human $U B E 3 A$ is overexpressed in glial cells (repo $>$ Dube $3 a$ ), not neurons $($ elav $>$ Dube3a), implicating glia in the etiology of Dup15q seizures (Hope, LeDoux et al. 
2017). Glial cells biallelically express Ube3a in mice (Yamasaki, Joh et al. 2003, Dindot, Antalffy et al. 2008, Judson, Sosa-Pagan et al. 2014, Grier, Carson et al. 2015), and GFAP positive astrocytes in the human brain similarly express UBE3A (Burette, Judson et al. 2018). Based on these observations, we propose that Dup15q individuals have elevated levels of UBE3A in glia and neurons. The contribution of glial cells, particularly astrocytes, to epilepsy is becoming more apparent (Coulter and Steinhauser 2015), and increased UBE3A levels in human glia may cause the underlying molecular deficits that lead to seizures in Dup15q.

Previously, we identified reduced levels of the $\mathrm{Na}^{+} / \mathrm{K}^{+}$exchanger ATP $\alpha$ in our glial Dube3a overexpression seizure model that appeared to drive a large portion, but not all, of the seizure phenotype (Hope, LeDoux et al. 2017). As such, further characterization of repo $>$ Dube 3 a seizure sensitive flies was needed. In this study we employed whole transcriptome analysis through RNA-sequencing and whole proteome analysis through liquid chromatography coupled to high-resolution mass spectrometry of whole fly head extracts in order to identify the underlying changes in glial or neuronal Dube $3 a$ overexpression. By combining both transcript and protein profiling, we were able to identify genes that were altered at either the transcript level, the protein level, or both the transcript and protein level based on Dube3a cell-type-specific overexpression.

\section{Methods}

\section{Fly Stocks}

Flies were reared on standard corn meal media (Bloomington Stock Center) and maintained on a 12-hour light/dark cycle at $25^{\circ} \mathrm{C}$. repo-GAL4 was obtained from the Bloomington Drosophila Stock Center (BDSC \# 7415), elav-GAL4 was provided by Dr. Hugo Bellen (Baylor College of Medicine), and UAS-Dube3a was described previously (Reiter, Seagroves et al. 2006). The GSTD1-LacZ reporter line was provided by Mel Feany and Dirk Bohmann and has been described previously (Sykiotis and Bohmann 2008).

The following stocks were used for gliopathic seizure screening: $\mathrm{P}(\mathrm{Cary})$ attP2 (UAS-RNAi Control, BDSC \#36303), UAS-Comt-RNAi (BDSC \#31470), UAS-ComtRNAi (BDSC \#31666), UAS-Comt-RNAi (BDSC \#34913), UAS-eas-RNAi (BDSC \#38528), UAS- $A T P \alpha-$ RNAi (BDSC \#32913), UAS- $p k$-RNAi (BDSC \#32413), UASSesB-RNAi (BDSC \#31230), UAS-SesB-RNAi (BDSC \#31077), UAS-Tko-RNAi (BDSC \#38251), UAS-porin-RNAi (BDSC \#67873), UAS-porin-RNAi (BDSC \#29572), UASsyn-RNAi (BDSC \#27304), UAS-Sod2-RNAi (BDSC \#25969), UAS-Sod2-RNAi (BDSC \#36871), UAS-Sod2-RNAi (BDSC \#32496), UAS-mt:ATPase6-RNAi (BDSC \#60435), UAS- $m t$ :ATPase6-RNAi (BDSC \#55297), UAS-TetxLC (BDSC \#28997), UAShtt.128Q.FL (BDSC \#33808), UAS-Jbug-RNAi (BDSC\#39070), UAS-Jbug-RNAi (BDSC \#31590), UAS-Sirup-RNAi (BDSC \#43213), UAS-Ttc 19-RNAi (BDSC \#64958), UAS-YME1L-RNAi (BDSC \#31752), UAS-levy-RNAi (BDSC \#67789), UAS-ST6GAL- 
RNAi (BDSC \#44528), UAS-ST6GAL-RNAi (BDSC \#53907), UAS-ST6GAL-RNAi (BSDC \#65876), UAS-ncc69-RNAi (BDSC \#28682), UAS-atl-RNAi (BDSC \#36736), UAS-COX7A-RNAi (BDSC \#57572), UAS-Tpi-RNAi (BDSC \#51829), UAS-Tpi-RNAi (BDSC \#26304), UAS-sra-RNAi (BDSC \#27260), UAS-sra-RNAi (BDSC \#36900), UAS- $k c c$-RNAi (BDSC \#34584), UAS- $k d n$-RNAi (BDSC \#36740), UAS-cac-RNAi (BDSC \#27244), UAS-Cpo-RNAi (BDSC \#28360), UAS-Cpo-RNAi (BDSC \#60388), UAS-GFAP (BDSC \#64368), UAS-zyd-RNAi (BDSC \#25851), UAS-stai-RNAi (BDSC \#53925), UAS-stai-RNAi (BDSC \#36902), UAS-Para-RNAi (BDSC \#31676), UASPara-RNAi (BDSC \#33923), UAS-Para-RNAi (BDSC \#31471), UAS-Letm1-RNAi (BDSC \#37502), UAS-nrv2-RNAi (BDSC \#28666), UAS-nrv2-RNAi (BDSC \#37495), UAS-CG9657-RNAi (BDSC \#28384), UAS-sei-RNAi (BDSC \#31681), and UAS-SeiRNAi (BDSC \#31682).

\section{RNAsequencing}

RNA was isolated from control (UAS-Dube3a alone), glial Dube3a overexpression (repo $>$ Dube $3 a$ ), or neuronal overexpression of Dube $3 a$ (elav $>$ Dube $3 a)$ flies by removing 15-25 fly heads per genotype and homogenizing in TRI Reagent (Applied Biosystems). Total RNA was extracted with Directzol RNA MiniPrep Plus (Zymo Research Corp) according to the manufacturer's instructions which included a DNAse treatment step. Purity and concentration was verified using NanoDrop spectrophotometer (ThermoFisher Scientific), and integrity was confirmed using a Bioanalyzer (Agilent). Each genotype was run in triplicate. RNAseq was performed by PGM sequencing at the UTHSC Molecular Resource Center (MRC). Each RNA sample was converted to ion torrent sequencing libraries and run on $318 \mathrm{v} 2$ chips using an Ion Torrent Proton sequencer. All groups and replicates were run simultaneously to eliminate chip-to-chip variability.

\section{RNAseq Analysis}

At least 35M unmapped reads we available per replicate sample for three groups and three replicates (1) UAS-Dube 3a, (2) repo $>$ Dube3a, (3) elav $>$ Dube3a. RNAseq alignments and analysis was performed using a local Slipstream appliance running a GALAXY installation. All FASTQ files were gathered from the sequencer. Quality assurance was performed using FASTQC. All reads were trimmed to remove any nucleotide that fails a phred score $<$ Q20. The trimmed FASTQ files were aligned to the reference library using RNA STAR. Once aligned, the SAM files were collected and mined for read count information of each gene present in the reference file. Read counts were normalized using Transcripts per Million (TPM) method across the entire experiment. The resulting data was analyzed with DeSeq2. All genes that failed to yield at least a 1.5 -fold change and a $p$-value greater than 0.05 were removed. Benjamini and Hochberg false discovery rate was performed on the trimmed gene list. All genes that failed to yield a false discovery rate $<0.05$ were removed. The final significant differential gene list were loaded into $\mathrm{R}$ for visualization. 


\section{Quantitative Proteomics}

Protein was extracted from fly heads by homogenizing 30-40 fly heads of each genotype in RIPA buffer plus complete Protease Inhibitor Cocktail (Roche). Lysates were de-salted and immobilized using a Filter Aided Sample Preparation (FASP) column followed by trypsin digestion and Tandem Mass Tag (TMT) labeling as described elsewhere (Jiang, Bomgarden et al. 2017). All groups were labeled at once to avoid variance in labeling reactions. The labeled digested peptides were then were analyzed by LC-MS/MS using an EASY-nLC 1000 UPLC system (Thermo Fisher Scientific San Jose, CA) with a $50 \mathrm{~cm}$ EASY-Spray Column coupled to an Orbitrap Fusion Lumos (Thermo Fisher Scientific, San Jose, CA) mass spectrometer. Separations were carried out using a $210 \mathrm{~min}$ gradient at a flow rate of $300 \mathrm{~nL} / \mathrm{min}$.

Post-acquisition analysis of raw MS data was performed with mass informatics platform Proteome Discoverer 2.0 and searched with the SEQUEST HT search engine. Precursor mass tolerance was set to $10 \mathrm{ppm}$. Fragment ion tolerance was $0.02 \mathrm{Da}$ when using the Orbitrap analyzer and 0.6 Da when using the ion trap analyzer. Carbamidomethylation on cysteine $(+57.021 \mathrm{Da})$ and TMT6 tags on $\mathrm{N}$ termini as well as lysine $(+229.163 \mathrm{Da})$ were set as static modifications. Dynamic modifications included oxidation on methionine $(+15.995 \mathrm{Da})$. Data were searched against a UniProt Drosophila melanogaster database. Peptide identification data was used for identification and quantification of corresponding parent proteins; Peptide abundance was calculated using signal to noise $(\mathrm{S} / \mathrm{N})$ values from corrected reporter ion abundances based on the manufacturer's data sheets. $\mathrm{S} / \mathrm{N}$ values where then log transformed and roll up at the protein level by summing the values for all the peptides to represent protein abundance. An ANOVA test was then performed to determine statistically relevant differences. For statistical validation of results, the FDR (False Discovery Rate) was determined and differences were considered statistically significant if FDR $<0.05$.

\section{Combined RNAseq and Proteomic Analysis}

RNAseq and proteomics data were combined into a single analysis by selecting proteins that were significantly differentially expressed that were also detected at the transcript level. Protein abundance ratios were used to calculate the protein $\log _{2}$ fold change and FPKM were used to calculate transcript $\log _{2}$ fold change.

\section{Quantitative Western Blot Analysis}

Protein was extracted from 30-40 fly heads by homogenization in RIPA buffer plus Complete Protease Inhibitor Cocktail (Roche). Samples were spun at 10,000 x g to remove exoskeleton. $20 \mu \mathrm{g}$ of protein was loaded into $1.5 \mathrm{~mm}$ NuPAGE Bis-Tris $4-12 \%$ gels (Invitrogen) and subsequently transferred to PVDF membranes (Millipore). 
Membranes were blocked with Odyssey Blocking Buffer (Li-Cor cat \# 927-50000). The following primary antibodies were diluted in Odyssey Blocking Buffer with $0.2 \%$ tween20: $\alpha$-GAPDH (1:5000, Abcam ab157156), $\alpha$-futsch (1:100, DSHB, 22C10), $\alpha$-synapsin (1:100, DSHB, 3C11), $\alpha$-Sap47 (1:100, DSHB, nc46), $\alpha$-Syx 1a (1:100, DSHB, 8C3), and $\alpha$-Nwk (1:1000, gift from J. Troy Littleton (Picowar Institute at MIT)). The following infrared secondary antibodies were diluted at 1:15,000 in Odyssey Blocking Buffer with $0.2 \%$ tween-20 and $0.01 \%$ SDS: $\alpha$-goat (Licor Cat\# 925-68074), $\alpha$-mouse (Licor Cat\# 926-32212), and $\alpha$-rabbit (Licor Cat\# 925-32211). Blots were imaged using an Odyssey Infrared Imaging System (Licor) and analyzed using Image Studio Lite (Licor) normalizing experimental bands to GAPDH as the loading control for calculating normalized adjusted relative signal intensities. All samples were run $\geq 3$ times to perform statistical analysis, and differences were considered statistically significant if $\mathrm{p}<0.05$.

\section{Seizure Susceptibility Assays}

Flies were collected on the day of eclosure under $\mathrm{CO}_{2}$ anesthesia and aged 3-5 days. On the day of testing flies were gently transferred by mouth pipette to empty vials. Flies were subjected to mechanical stress ("bang") by vortexing for $10 \mathrm{~s}$ on a standard laboratory vortexer (LabNet) at full speed. Recovery time was recorded for each fly as measured by time until the fly righted itself and was able to walk or groom and no longer displayed paralysis or uncontrolled muscle movements. To detect seizure sensitive flies, all experimental groups were compared to the control repo $>36303$-emptyvector-RNAi by one-way ANOVA followed by Dunnett's multiple comparison test. Groups were considered statistically significant if $\mathrm{p}<0.05$.

\section{Microarray Analysis}

Total RNA was isolated from control (repo $>36303$-emptyvector-RNAi) and gliopathic seizure lines by removing 15-25 fly heads per genotype and homogenizing in TRI Reagent (Applied Biosystems) and followed by RNA purification with Directzol RNA MiniPrep Plus (Zymo Research Corp) according to the manufacturer's instructions. Purity and concentration were verified using NanoDrop spectrophotometer (ThermoFisher Scientific) and Qubit (ThermoFisher Scientific). RNA integrity was measured using a Bioanalyzer (Agilent). mRNA from the gliopathic seizure flies was pooled into a single group by adding equal amounts of mRNA from each sample. Samples were run on a GeneChip Drosophila Gene 1.0 ST Array (ThermoFisher Scientific, \#902155).

CSV files were retrieved from UTHSC Molecular Resource Center after normalization performed by Affymetrix Expression Console. Quality assurance was checked against reference probes to ensure quality of data. Gene names, accession numbers, and expression were mined from each text file for each sample. All nonannotated information was removed from the file leaving only annotated gene expression. A Welsh $t$ test was run for pairwise interactions in order to obtain $p$-values for 
significance. Only genes with a p-value $<0.05$ were considered significant. The mean, variance, standard deviation, and fold change were calculated for each pairwise comparison. Benjamini Hochberg false discovery rate method was applied in order to obtain the adjusted $p$-value for each gene. Only genes with an adjusted p-value $<0.05$ were considered significant.

\section{Quantitative Real-time Polymerase Chain Reaction (qRT-PCR)}

mRNA was converted to cDNA using a Transcriptor First Strand cDNA Synthesis Kit (Roche, 04379012001) according to the manufacturer's instructions. Briefly, $500 \mathrm{ng}$ of total mRNA was used per genotype with random hexamer primers for cDNA synthesis. Probes were selected and primers were designed using the Universal Probe Library Assay Design Center software (Roche). The following primers were used with probe \#61 from the Universal Probe Library (UPL) for GstS1: F 5'AAGGACAACGATGGTCACCT-3', R 5'-GATGCCTGCGAAGTAGACG-3'. For GstD2, the following primers were used with UPL probe \#5: F: 5'TTCTCGACACCTTCCTGGAG-3', R 5'-AGTGGACAGGATGGCAATGT-3'. For GstE7, the following primers were used with UPL probe \#25: F: 5'AATACGGCAAAACGGACAGT-3', R 5'-AATCGCTGATCCACGACAG-3'. For GstD5, the following primers were used with UPL probe \#55: F: 5 'CCACCTCACAGTGGCTGATA-3', R 5'-TCAAAGATTTCGAAAGTGGAAAC-3'. For $t b p$, the following primers were used with UPL probe \#109: F: 5'ACAGGGGCAAAGAGTGAGG-3', R 5'-CTTAAAGTCGAGGAACTTTGCAG-3'.

All assays were performed in triplicate for each cDNA sample. Cycling conditions of the Roche LC480 were: $95^{\circ} \mathrm{C}$ for $5 \mathrm{~m}$ followed by 40 cycles of $95^{\circ} \mathrm{C}$ for 10 $\mathrm{s}, 60^{\circ} \mathrm{C}$ for $30 \mathrm{~s}$, and $72^{\circ} \mathrm{C}$ for $10 \mathrm{~s}$ during which fluorescence was measured. Crossing point $(\mathrm{Cp})$ values were calculated for each sample using the absolute quantification algorithm (Roche), and average $\mathrm{Cp}$ values for three technical replicates were calculated. The average $\mathrm{Cp}$ values were normalized to TATA-binding protein ( $t b p$ ). $\log _{2}$ fold change was calculated by first finding the $\Delta \mathrm{Cp}$ for each sample, comparing experimental groups to the control group to calculate $\Delta \Delta \mathrm{Cp}$, calculating fold change with the equation $\mathrm{Fc}=$ $(2)^{-\Delta \Delta \mathrm{Cp}}$, and finally making the $\log _{2}$ transformation.

\section{Immunohistochemistry and Image Acquisition}

Flies were anesthetized with $\mathrm{CO}_{2}$ and brains were dissected in phosphate buffered saline (PBS) and fixed with 4\% formaldehyde for 1 hour. Brains were blocked and permeabilized with $5 \%$ bovine serum albumin and $0.1 \%$ Triton $X-100$. Primary antibodies used were rabbit $\alpha-\beta$ Gal (1:100, Thermofisher Scientific 14-6773-81) and mouse $\alpha$-repo (1:100, DSHB 8D12), incubated overnight at $4^{\circ} \mathrm{C}$ in the same blocking solution. Secondary antibodies used were donkey $\alpha$-rabbit 594 (1:500, Thermofisher Scientific A21207) and goat $\alpha$-mouse 488 (1:500, Thermofisher Scientific A11029) and 
were incubated for 1 hour at room temperature. Slides were mounted with FluoromountG mounting media (SouthernBiotech, 0100-20) and sealed with clear nail polish.

Confocal images were acquired using a Zeiss 710 laser scanning confocal microscope (Zeiss) located in the UTHSC Neuroscience Institute Imaging Core. Images were captured at $1024 \times 1024$ resolution with a $63 \mathrm{X}$ objective lens at $1 \mu \mathrm{M} Z$-section thickness. Detector gain and offset were optimized for the control group and settings were kept consistent across treatment groups to allow for comparisons between groups.

\section{Feeding Flies Picrotoxin (PTX)}

A $50 \mathrm{mM}$ stock solution of PTX (Acros Organics) was made in DMSO. Flies were fed $2.5 \mathrm{mM}$ PTX in 5\% sucrose (Sigma) with $1 \%$ green food color by placing flies in a vial containing a Kimwipe saturated with PTX feeding solution. Flies were left to feed for 24 hours, and consumption was confirmed by the presence of green dye in their abdomens. Control flies were fed DMSO alone.

\section{Data Analysis}

Data analysis and statistical tests were performed using Prism 6.0 (GraphPad). To determine differences between multiple samples, one-way ANOVA was used with Tukey's multiple comparisons ( $\alpha$ was set to 0.05 ). Heatmaps were generated using RStudio and figures were generated in Adobe Illustrator (Adobe).

\section{Results}

\section{Transcriptomic Profiling Reveals an Upregulation of Glutathione S-transferases in repo $>$ Dube3a Fly Heads}

We previously demonstrated that repo $>$ Dube $3 a$ flies overexpressing Dube $3 a$ in all glial cells display a severe bang-sensitive seizure phenotype, while elav $>$ Dube $3 a$ flies expressing Dube3a in all neurons do not (Hope, LeDoux et al. 2017). In order to investigate gene expression changes in these animals at the transcript level we performed RNAseq using RNA extracted from whole fly heads in control UAS-Dube $3 a$ alone, glial Dube $3 a$ overexpression repo $>$ Dube $3 a$, and neuronal Dube $3 a$ overexpression elav $>$ Dube $3 a$ flies. We reliably identified and quantified transcripts from approximately 11,000 genes across all three groups. We found 851 differentially expressed genes in repo $>$ Dube3a compared to UAS-Dube3a controls and 495 differentially expressed genes in elav $>$ Dube $3 a$ compared to controls (p-adjusted $<0.05$ ). Of these differentially expressed transcripts, 180 were shared between repo $>$ Dube $3 a$ and elav $>$ Dube $3 a$ (Figure 5-1A, Supplemental RNAseq Data). 
A

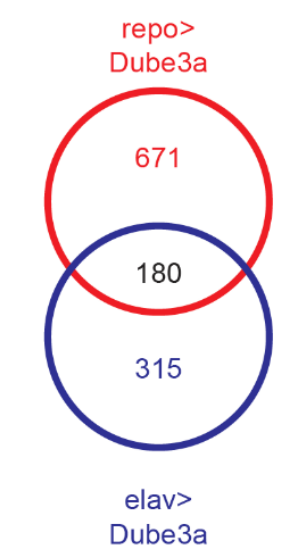

C

Glutathione S-transferase Enrichment

repo $>$ Dube $3 a$ elav>Dube3a

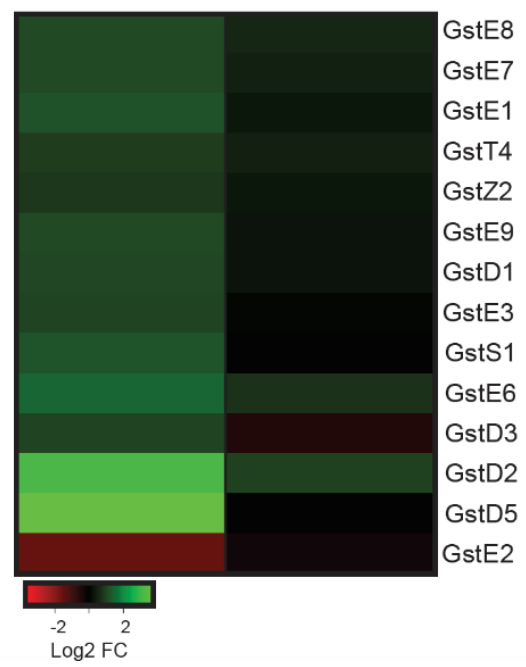

B

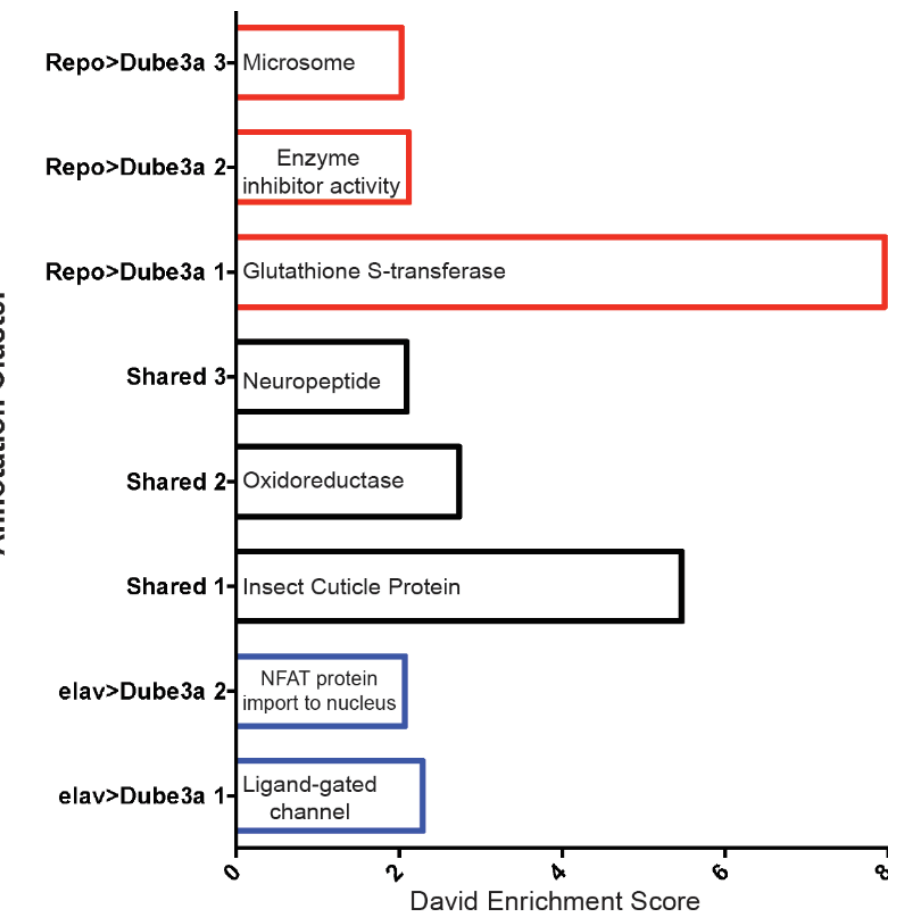

D

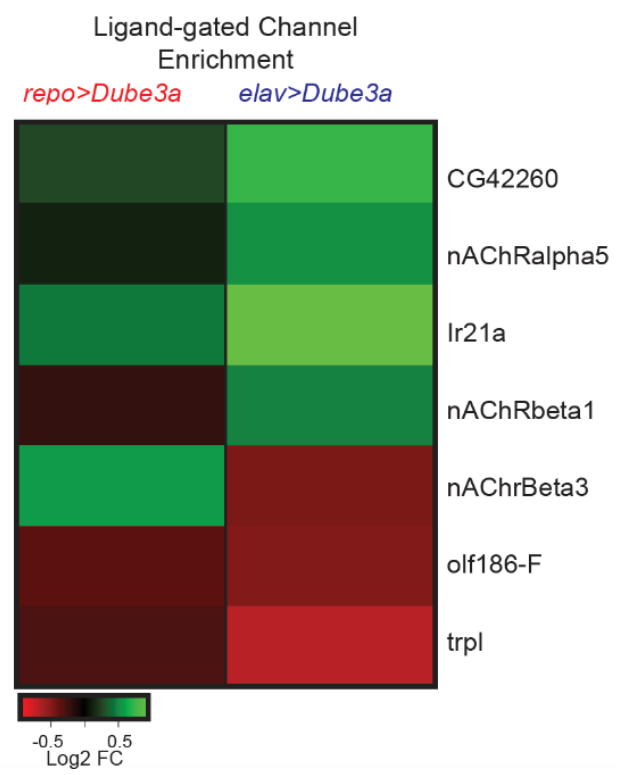

Figure 5-1. Transcript profiling reveals an upregulation of GST genes in repo $>$ Dube $3 a$ flies.

A) Venn diagram of differentially expressed transcripts between repo $>$ Dube $3 a$ and elav $>$ Dube $3 a$ compared to UAS-Dube3a controls. B) DAVID enrichment groups for differentially expressed transcripts in each portion of the Venn diagram. C) Heatmap indicating an upregulation of 13/14 GST genes in repo $>$ Dube $3 a$. This upregulation was not observed in neuronal elav $>$ Dube3a overexpression. D) Heatmap illustrating the differential expression for the "ligand-gated channel" enrichment group observed in elav $>$ Dube3a. 
To identify biological enrichments within each portion of the Venn diagram, we utilized the Database for Annotation, Visualization, and Integrated Discovery (Huang da, Sherman et al. 2009, Huang da, Sherman et al. 2009), and plotted DAVID analysis enrichment groups with an enrichment score (ES) greater than 2 for each group (Figure 5-1B). In the 180 differentially expressed genes shared between repo $>$ Dube $3 a$ and elav $>$ Dube $3 a$, we observed an enrichment in "insect cuticle proteins" $(\mathrm{ES}=5.46)$, "oxidoreductase" (ES = 2.73), and "neuropeptide" $(\mathrm{ES}=2.09)$, (Fig. 1B). Within the 671 repo $>$ Dube3a specific differentially expressed genes we found the strongest enrichment in "glutathione S-transferase" $(\mathrm{ES}=7.96)$. For the 315 elav $>$ Dube $3 a$ specific significantly differentially expressed transcripts we observed an enrichment in "ligandgated channel activity" ( $\mathrm{ES}=2.29)$ and "positive regulation of NFAT protein import into nucleus" (ES = 2.07) (Figure 5-1B, for all enrichment groups see Supplemental RNAseq Data). We plotted the $\log _{2}$ fold change for GST enrichment observed in repo $>$ Dube $3 a$ for repo $>$ Dube $3 a$ and elav $>$ Dube $3 a$ compared to controls and observed an upregulation of 13/14 GST genes and a downregulation of GstE2 in repo $>$ Dube3a that remained largely unchanged in elav $>$ Dube $3 a$ (Figure 5-1C). We also plotted the $\log _{2}$ fold change for the ligand-gated channel enrichment from elav $>D u b e 3 a$ for repo $>$ Dube $3 a$ and elav $>$ Dube $3 a$ compared to controls (Figure 5-1D). These results indicate that overexpression of Dube3a in glial cells has a stronger impact on the global transcriptome compared to neuronal Dube $3 a$ overexpression. Additionally, overexpression of Dube $3 a$ in glia causes both seizures and an upregulation of GST genes, and the upregulation of GSTs within glial cells occurs in idiopathic treatment resistant epilepsy (Shang, Liu et al. 2008).

\section{Proteomic Profiling Reveals Differential Expression of Neurotransmitter Secretion Proteins in repo $>$ Dube3a Fly Heads}

Next, we investigated global changes at the protein level using quantitative proteomics through mass spectrometry. We reliably identified and quantified approximately 2,500 proteins across each sample. 364 proteins were differentially expressed (FDR $<0.05$ ) in repo $>$ Dube $3 a$ compared to control UAS-Dube $3 a$, and 137 were differentially expressed (FDR $<0.05$ ) in elav $>$ Dube $3 a$ compared to UAS-Dube $3 a$ flies. Of these differentially expressed proteins, 85 were shared between repo $>$ Dube $3 a$ and elav $>$ Dube $3 a$ (Figure 5-2A, see Supplemental Proteomics Data). The 85 shared differentially expressed proteins were significantly enriched in three groups with an ES $>$ 2.0; "muscle cell development" (ES = 4.58), "Sulfation" (ES = 2.55), and "Actin" (ES = 2.72) (Fig. 2B, Supplemental Table 4). The enrichment of the GO term "muscle cell development" was initially concerning, suggesting possible contamination in the protein prep. The proteins included in the enrichment group are futsch, wupa, mf, up, act88f, and prm, all of which were detected in proteomic analysis of adult Drosophila heads in previous studies (Aradska, Bulat et al. 2015). Additionally, futsch is found at the neuromuscular junction (NMJ) causing futsch to be included in the GO term "muscle cell development", however futsch is known to be expressed from the neuronal side at the NMJ and is expressed widely throughout the central nervous system (Hummel, Krukkert 
A

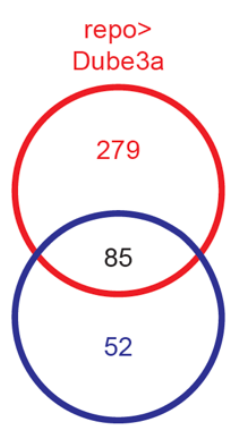

elav>

Dube3a
B

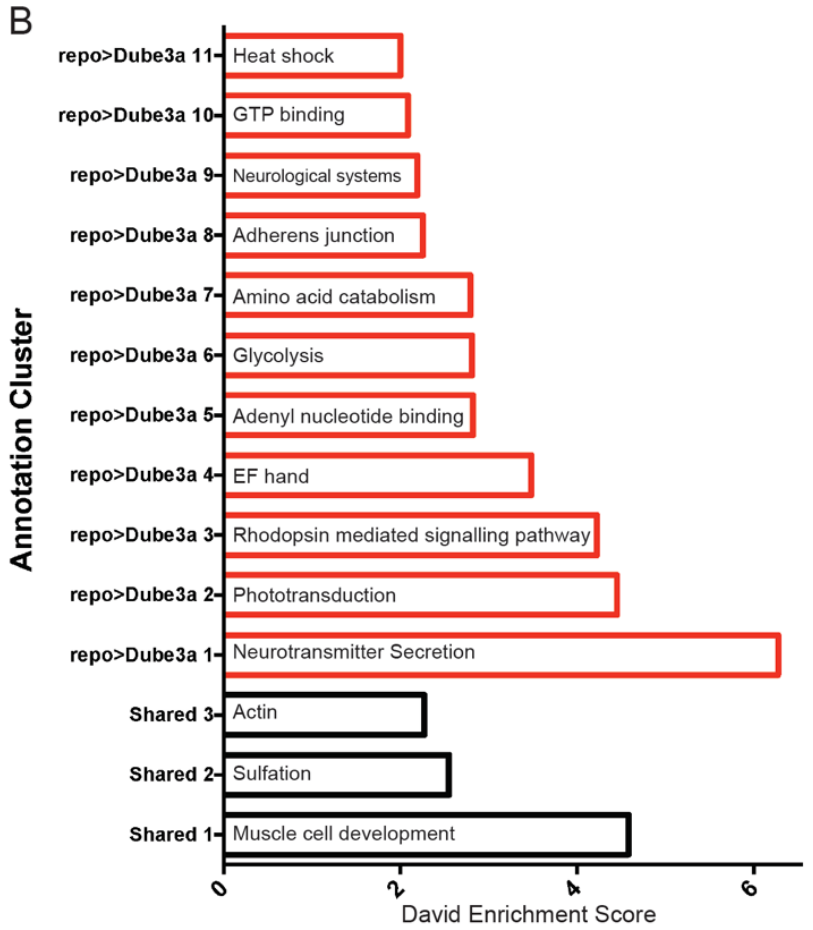

$\mathrm{D}$

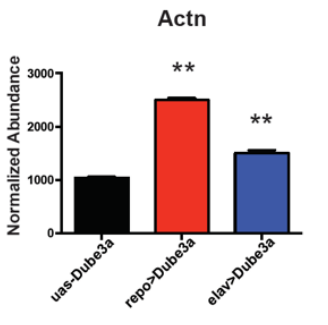

Apolipophorins (Rfabg)

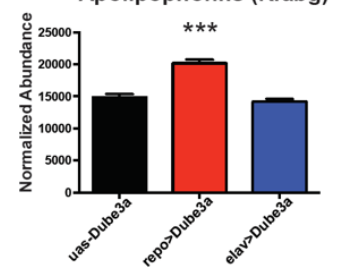

Actin-57B
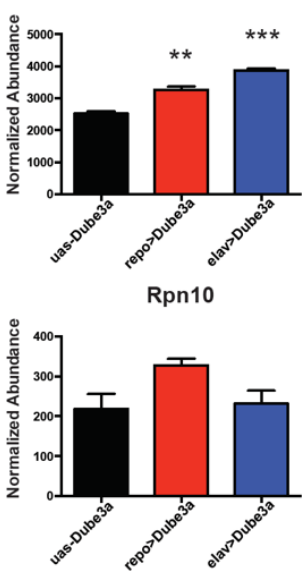

Figure 5-2. Proteomic profiling reveals differential expression of neurotransmitter secretion proteins in repo $>$ Dube $3 a$ flies.

A) Venn diagram of differentially expressed proteins between repo $>$ Dube $3 a$ and elav $>$ Dube3a. B) DAVID enrichment groups with an enrichment score greater than 2 for differentially expressed proteins for each portion of the Venn diagram. C) Normalized abundance ratios for the two detected isoforms of the $\mathrm{Na}^{+} / \mathrm{K}^{+}$ATPase ATP $\alpha$ following overexpression of Dube $3 a$ in glia or neurons compared to controls indicates downregulation of both isoforms only in repo $>$ Dube3a fly heads. D) Normalized abundance ratios for proteins previously reported to be regulated in a Dube3a dependent manner. Error bars represent S.E.M, and $*=\mathrm{FDR}<0.05, * *=\mathrm{FDR}<0.01$, and $* * *=$ FDR $<0.005$. 
et al. 2000), alleviating our concerns. The 279 repo $>$ Dube $3 a$ specific differentially expressed proteins were significantly enriched in 11 groups with an ES $>2.0$, the top two being "neurotransmitter secretion" $(\mathrm{ES}=6.27)$ and "phototransduction" $(\mathrm{ES}=4.45)$

(Figure 5-2B, Supplemental Proteomics Data). Within the 52 elav $>$ Dube 3 a specific differentially expressed proteins, no enrichment group was observed with an ES > 2.0, however the top enrichment was "stress response" $(E S=1.96)$. Similar to our RNAseq data, these results suggest that overexpression of Dube3a in glia causes a larger change in the global proteomic landscape of the fly brain compared to overexpression of Dube3a in neurons. Additionally, we observed an enrichment in the GO term "neurotransmitter secretion" and "phototransduction" which are predominantly neuronal processes, suggesting cell non-autonomous effects of glial Dube3a overexpression in repo $>$ Dube $3 a$ flies.

We previously reported downregulation of ATP $\alpha$ upon overexpression of Dube $3 a$ in glial cells (Hope, LeDoux et al. 2017) and that ATP $\alpha$ can be ubiquitinated by Dube3a (Jensen, Farook et al. 2013). We detected two peptides that mapped to two different isoforms of ATP $\alpha$ and we observed significantly reduced ATP $\alpha$ levels in the repo $>$ Dube $3 a$ group for both ATP $\alpha$ accession P13607 ( $<<0.0005)$ and for ATP $\alpha$ accession E1JIR4 $(\mathrm{p}<0.01)$, however we did not observe significant reductions in either isoform of ATP $\alpha$ in elav $>$ Dube $3 a$ flies (Figure 5-2C). These results confirm our prior findings that elevated levels of Dube $3 a$ in glial cells results in reduced levels of ATP $\alpha$ protein (Hope, LeDoux et al. 2017). Additionally, we checked the levels of other proteins found to be influenced by Dube3a. Actin, Actin-57B, and apolipophorins were previously reported to be upregulated following elevated levels of Dube3a protein by our group (Jensen, Farook et al. 2013). Here we observed an upregulation of both Actin and Actin57B in both repo $>$ Dube $3 a$ and elav $>$ Dube $3 a$, and apolipophorins was only upregulated in repo $>$ Dube $3 a$ (Figure 5-2D), confirming our previous proteomics results (Jensen et al., 2013). Rpn10 has been reported as a direct Ube3a substrate (Lee, Ramirez et al. 2014), however we detected no significant difference in Rpn10 levels in elav $>$ Dube3a or repo $>$ Dube $3 a$, with levels slightly, but not significantly, increased in the repo $>$ Dube3a group (Figure 5-2D).

\section{Comparison of the Transcriptome and the Proteome Reveals Downregulation of Synaptic Transmission Proteins in repo $>$ Dube3a Fly Heads}

We compared the proteome and the transcriptome to determine whether expression changes at the protein level could be explained by changes at the transcript level. We selected all differentially expressed proteins that were also detected at the transcript level and mapped the $\log _{2}$ fold change of both the transcript and protein for repo $>$ Dube $3 a$ compared to UAS-Dube $3 a$ (Figure 5-3A, Supplemental Repo Quadrant Analysis) and for elav>Dube $3 a$ compared to UAS-Dube $a$ (Figure 5-3B, Supplemental 

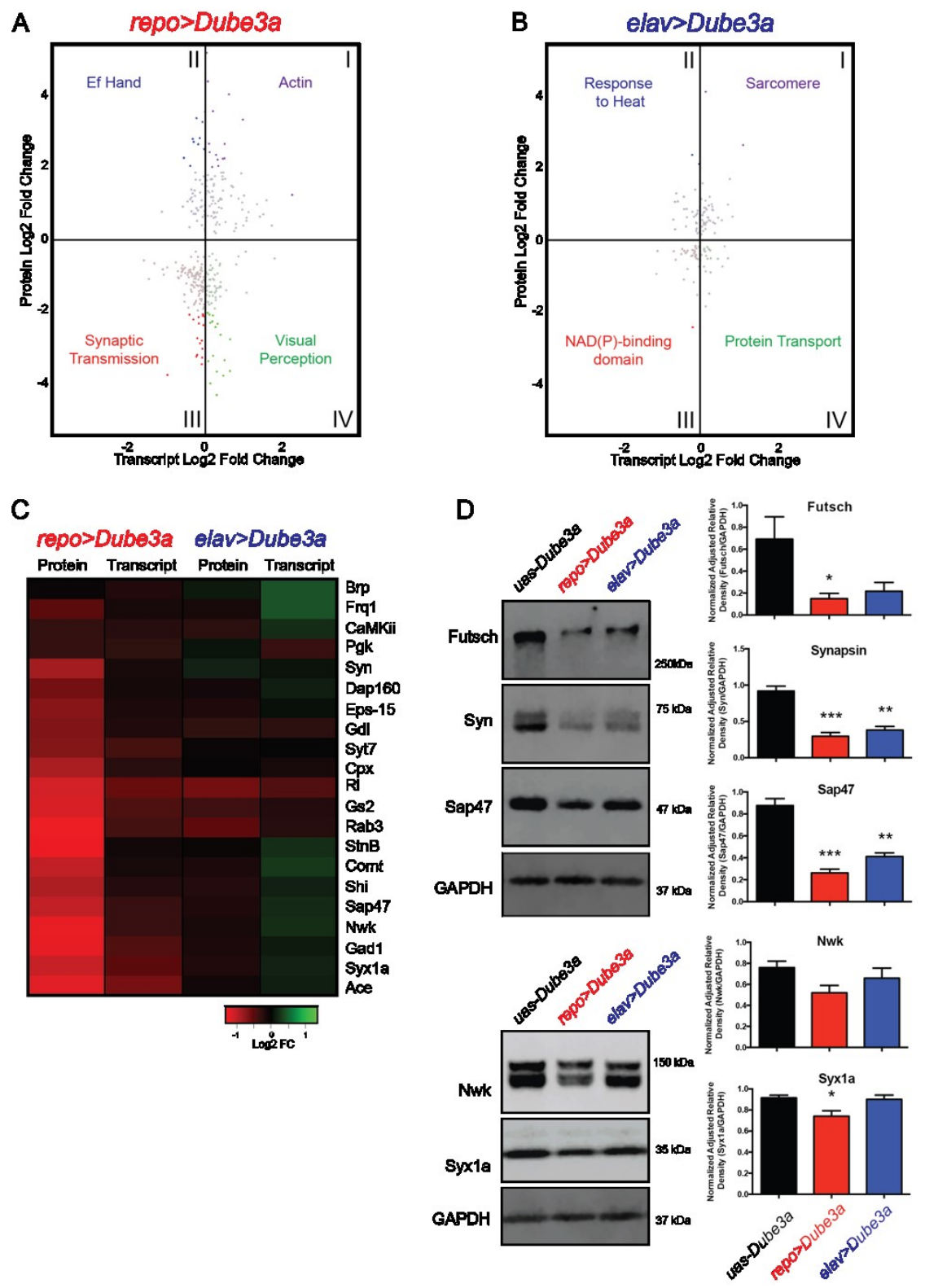

Figure 5-3. Cell non-autonomous downregulation of synaptic transmission proteins in repo $>$ Dube $3 a$ flies.

A) $\log _{2}$ fold change of significantly differentially expressed proteins also detected at the transcript and protein level in repo $>$ Dube $3 a$ and B) $\log _{2}$ fold change of significantly differentially expressed proteins also detected at the transcript level. The top DAVID enrichment group is stated in each quadrant of the graph. C) Heatmap of genes at the transcript and protein level for the "synaptic transmission" enrichment group in both repo $>$ Dube $3 a$ and elav $>$ Dube3a. Note the cell non-autonomous downregulation observed for neuronal proteins in repo $>$ Dube3a brain. D) Quantitative IR Western blot confirmation of "synaptic transmission" protein downregulation in both repo $>$ Dube $3 a$ and elav $>$ Dube $3 a$. $\mathrm{N} \geq 3$ for each genotype, error bars represent S.E.M., and $*=\mathrm{p}<$ $0.05, * *=\mathrm{p}<0.01$, and $* * *=\mathrm{p}<0.005$. 
Elav Quadrant Analysis). Quadrants I, II, III, and IV represent genes upregulated at both the protein and transcript level, genes upregulated at the protein level but downregulated at the transcript level, genes downregulated at both the protein and transcript level, and genes downregulated at the protein level but upregulated at the transcript level, respectively. In quadrant III of repo $>$ Dube3a we observed a significant DAVID enrichment in the GO term "synaptic transmission" (Enrichment Score = 15.7). This enrichment included 21 genes, and we plotted $\log _{2}$ fold change values for each gene at the protein and transcript level in both repo $>$ Dube $3 a$ and elav $>$ Dube $3 a$ (Figure 5-3C) all of which are downregulated in repo $>$ Dube $3 a$ but relatively unchanged in elav $>$ Dube $3 a$ flies. These data suggest cell non-autonomous effects of Dube $3 a$ overexpression in glia, whereby glial elevation of Dube $3 a$ causes downregulation of neuronal synaptic transmission proteins.

Next, we tried to validate the downregulation of synaptic proteins observed through RNAseq and proteomics via western blot (Figure 5-3D). We observed a significant effect of genotype on synapsin levels (One-way ANOVA, $\mathrm{F}_{(2,6)}=35.91$, $\mathrm{p}<$ 0.005 ), a vesicle protein that regulates neurotransmitter release (Winther, Vorontsova et al. 2015), and found that synapsin is downregulated at the protein level in repo $>$ Dube $3 a$ compared to control UAS-Dube3a flies (Tukey's multiple comparisons; $\mathrm{p}<0.005$ ). We found a downregulation of synapsin in elav $>$ Dube $3 a$ compared to UAS-Dube $3 a$ flies (Tukey's multiple comparisons; $p<0.01$ ) that was not detected during our proteomic analysis. In addition to synapsin, we confirmed the downregulation of Sap47, a vesicle associated protein (Saumweber, Weyhersmuller et al. 2011), at the protein level with genotype displaying a significant effect on Sap47 levels (One-way ANOVA, $\mathrm{F}_{(2,6)}=$ $49.38, \mathrm{p}<0.005)$. We observed reduced Sap47 levels in repo $>$ Dube3a compared to UAS-Dube3a flies (Tukey's multiple comparisons; $\mathrm{p}<0.005$ ) and in elav $>$ Dube $3 a$ flies (Tukey's multiple comparisons; $\mathrm{p}<0.005$ ). Although not detected in our proteomics dataset, we tested levels of the neuronal microtubule protein futsch and detected a significant downregulation of futsch protein levels in repo $>$ Dube $3 a$ compared to UASDube $3 a$ alone (One-way ANOVA, $\mathrm{F}_{(2,6)}=5.18, \mathrm{p}<0.05$, Tukey's multiple comparisons; $\mathrm{p}<0.05$ ). Syx1a mediates vesicle fusion (Schulze, Broadie et al. 1995), and Syx1a protein expression was also dependent upon genotype (One-way ANOVA, $\mathrm{F}_{(2,21)}=5.75$, $\mathrm{p}<0.05)$, and levels were significantly reduced only in repo $>$ Dube $3 a$ compared to UASDube3a controls (Tukey's multiple comparisons, $\mathrm{p}<0.05$ ). We also tested levels of Nwk, a protein that regulates synapse morphology and actin dynamics (Rodal, Motola-Barnes et al. 2008), however there was slightly more variation and no significant differences were detected between genotypes (One-way ANOVA, $\mathrm{F}_{(2,21)}=2.43, \mathrm{p}=0.11$ ). Taken as a whole, our Western blot data verifies the results from our proteomics data that in general, synaptic proteins are downregulated in repo $>$ Dube $3 a$ flies in a cell non-autonomous manner as we are manipulating Dube3a expression in glial cells.

\section{Glutathione S-transferase Upregulation is Common Among Gliopathic Seizure Flies}

We wanted to know whether any of the molecular changes that occur in our Dup15q fly model of epilepsy were common to other epilepsies studied in flies and if 
these "bang sensitive" genes had anything to do with glial cells. We identified and selected 54 different UAS lines that covered 34 genes which were associated with the term "bang sensitive" in FlyBase and crossed these UAS-RNAi or UAS- $c D N A$ lines to repo-GAL4 to manipulate gene expression within glial cells. Lethality was observed in 6 of these lines, including UAS-porin-RNAi (BDSC \#67873), UAS-TetxLC (BDSC \#28997), UAS-Jbug-RNAi (BDSC \#39070), UAS-Zyd-RNAi (BDSC \#25851), UASLetm 1-RNAi (BDSC \#37502), and UAS-nrv2-RNAi (BDSC \#28666). We identified 6 additional lines that cause a bang sensitive seizure phenotype when crossed to repoGAL4 (Figure 5-4A, One-Way ANOVA, $\mathrm{F}_{(48,532)}=46.17, \mathrm{p}<0.0001$; Dunnett's multiple comparison test $\mathrm{p}<0.05$ ). We called these flies "gliopathic" seizure flies because they have seizures driven by glial cell manipulations, most of which were RNAi knockdown of endogenous glial genes. Total head mRNA was collected from 5 of these gliopathic lines along with repo $>$ Dube3a, pooled into one group, and an mRNA microarray analysis was performed to look for shared transcriptional changes among all of the lines (repo $>C O X 7 A-R N A i$ was excluded from the microarray due to a less severe seizure phenotype, however it was included in subsequent qRT-PCR analysis). Upon comparing the gliopathic seizure group to a repo $>36303$-emptyvector-RNAi control line, we observed 120 significantly differentially expressed transcripts (FDR $<0.05$, Supplemental Microarray Data). DAVID analysis of these differentially expressed genes revealed a significant enrichment in the GO term "Detoxification" (ES = 2.45), comprising the GSTs GSTD2, GSTD5, and GSTS1. Additionally, we observed an enrichment in "glutathione S-transferase activity" $(\mathrm{ES}=2.31)$, including the additional gene GSTE7 (Supplemental Microarray Data). Upon performing qRT-PCR for GSTS1, GSTD2, GSTE7, and GSTD5 on each individual line, we confirmed an upregulation of these GST transcripts in 5 out of the 7 gliopathic seizure lines (Figure 5-4B). These data indicate that GST upregulation is a common occurrence in gliopathic seizure and is not a phenomenon specific to our fly model of Dup15q epilepsy.

To determine whether the upregulation of GSTs occurred cell autonomously within glia or non-autonomously within some other cell type in the brain, such as neurons, we used a previously published GSTD1-LacZ reporter line (Sykiotis and Bohmann 2008). In control repo-GAL4;GSTD1-LacZ flies staining for $\alpha-\beta G a l$ was relatively diffuse (Figure 5-4C, top row). However, in repo $>$ Dube3a;GSTDI-LacZ seizure flies we observed a marked increase in $\alpha-\beta \mathrm{Gal}$ immunoreactivity which colocalized strongly with the glial-specific transcription factor repo (Figure 5-4C, bottom row). Finally, we asked whether inducing seizure-like activity alone through pharmacological means would similarly result in GST upregulation. We fed GSTD1$\mathrm{LacZ}$ flies $2.5 \mathrm{mM}$ picrotoxin (PTX) or DMSO (control) overnight to induce seizure-like behavior, and upregulation of GSTD1-LacZ did not occur PTX fed flies (Figure 5-5). Taken together, these data suggest that GST upregulation is common among gliopathic seizure flies and occurs cell autonomously within glial cells. 
A

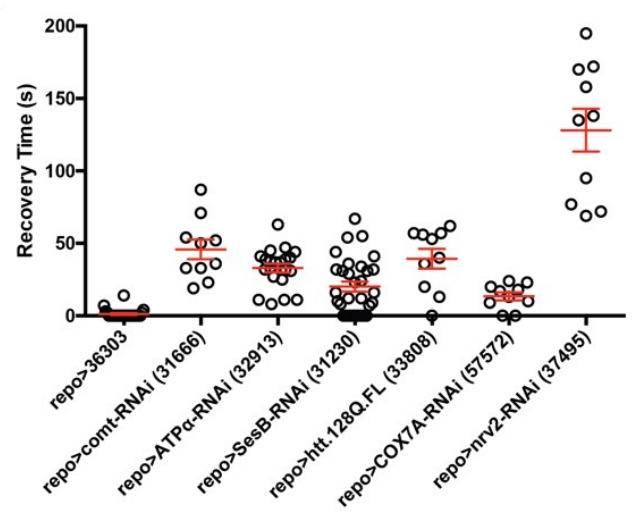

C

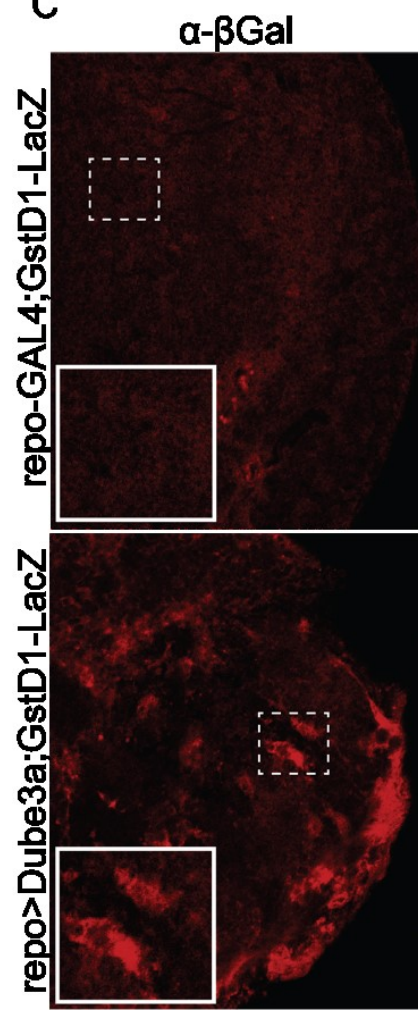

B

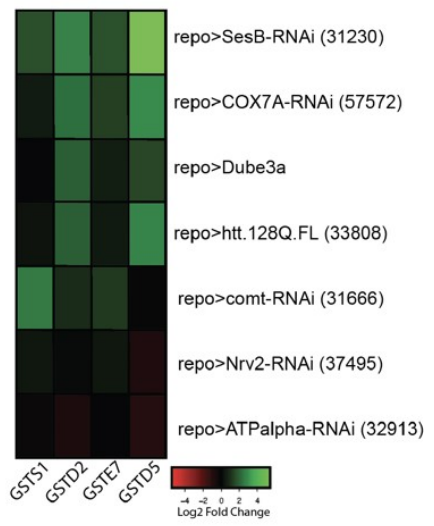

Merged
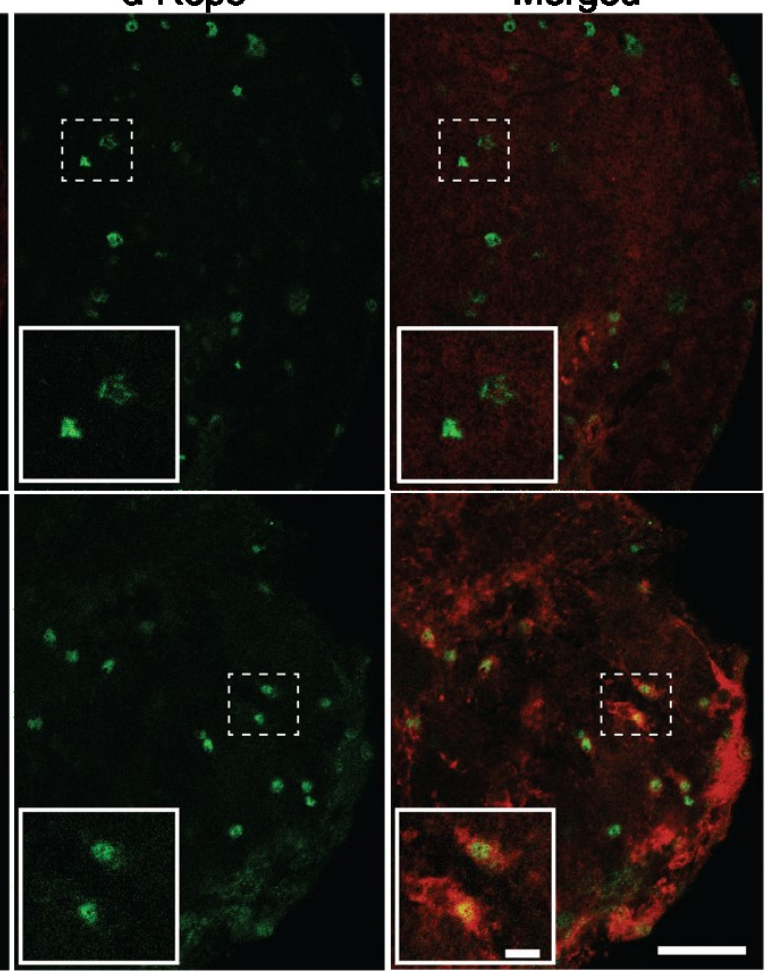

Figure 5-4. Glutathione S-transferase upregulation is common among multiple "gliopathic" seizure lines and occurs cell autonomously within glial cells.

A) Recovery times in the bang sensitivity seizure assay for 6 additional gliopathic seizure lines identified. B) Heatmap of glutathione S-transferase expression levels in each gliopathic seizure line indicates upregulation of glutathione S-transferases in 5/7 lines. Genes were originally identified by microarray as differentially expressed in a pooled gliopathic seizure group compared to a control group. C) Immunohistochemistry in repoGAL4;GstD1-LacZ or repo $>$ Dube $3 a ; G s t D 1-L a c Z$ for the glutathione S-transferase reporter GstDI-LacZ (red) reveals an upregulation of glutathione S-transferases cell autonomously within glial cells. All glial cells are marked with an $\alpha$-repo antibody (green). Error bars represent S.E.M. and scale bars represent $25 \mu \mathrm{m}$ or $5 \mu \mathrm{m}$ (inset). 


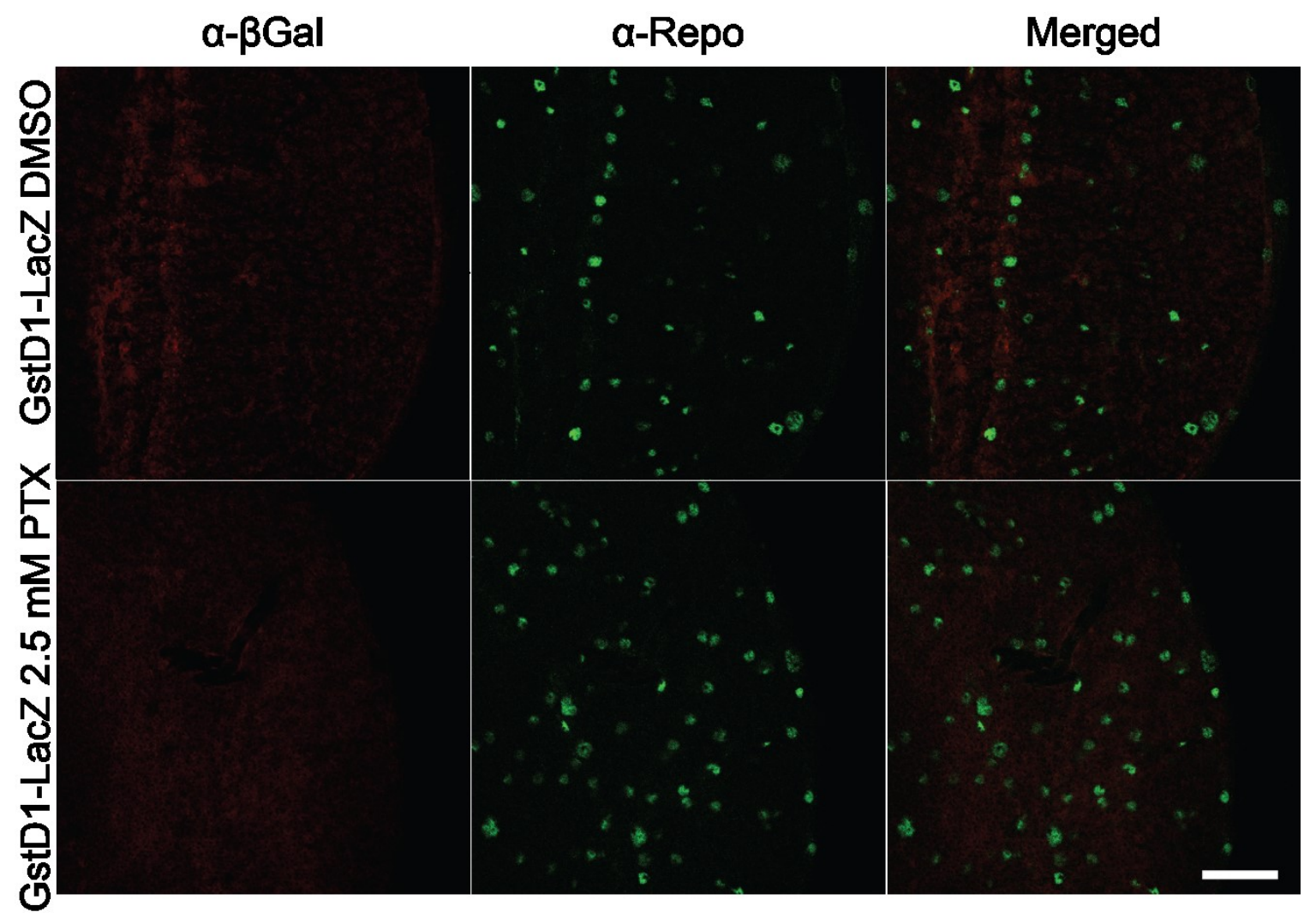

Figure 5-5. Glutathione S-transferase upregulation is not observed in flies fed PTX.

Overnight feeding of PTX (bottom row) did not induce expression of the GstD1-LacZ reporter (red) compared to DMSO control (top row). Glial cells are marked with $\alpha$-repo antibody (green). Scale bar is $25 \mu \mathrm{m}$. 


\section{Discussion}

In this study we show that glial Dube3a overexpression causes downregulation of synaptic transmission genes at the transcript and protein levels. We also demonstrate a cell autonomous upregulation of GSTs that was shared among multiple gliopathic seizure lines that we identified. In contrast, Dube3a overexpression in neurons resulted in fewer overall changes in the transcriptome and proteome, and fewer biologically relevant enrichment groups compared to Dube $3 a$ overexpression in glia. This study demonstrates the utility of Drosophila for identifying global changes in the transcriptome and proteome depending on the cell type overexpressing Dube $3 a$ (glia or neurons). We went on to show shared molecular changes across multiple genes causing gliopathic seizure.

Our proteomic experiments identified a sizeable portion of the Drosophila head proteome. While not exhaustive, we reliably measured peptides corresponding to 2,503 proteins. Recent efforts to completely identify the Drosophila head proteome revealed peptides corresponding to 4,559 proteins (Aradska, Bulat et al. 2015). Despite the lower coverage, we observed synaptic transmission protein downregulation in repo $>$ Dube $3 a$ flies which we confirmed via western blot. Importantly, these experiments were not designed to detect direct ubiquitin ligase substrates of Dube $3 a$, only the consequences of Dube $3 a$ overexpression in different cell types. As such, the changes observed at the protein level could be secondary, tertiary, or even quaternary effects due to Dube $3 a$ overexpression. We observed an enrichment of downregulated proteins in the gene ontology term "synaptic transmission" in repo $>$ Dube3a flies, suggesting cell nonautonomous effects of Dube $3 a$ overexpression in glia. Some of these genes are expressed in glia and contribute to the synaptic transmission process, such as glutamine synthetase 2 (Gs2) which converts glutamate to glutamine (Sinakevitch, Grau et al. 2010). However, genes including futsch (Hummel, Krukkert et al. 2000), synapsin (Oland, Biebelhausen et al. 2008), Sap47 (Reichmuth, Becker et al. 1995, Saumweber, Weyhersmuller et al. 2011), bruchpilot (Wagh, Rasse et al. 2006), and nervous wreck (Rodal, Motola-Barnes et al. 2008) are predominantly expressed in neurons. Therefore, our results show that repo $>$ Dube $3 a$ has a cell non-autonomous effect on gene expression in neurons.

Human orthologs of some of the synaptic transmission proteins downregulated in repo $>$ Dube $3 a$ flies are associated with human epilepsy. Nonsense variants in Synapsin I, the human ortholog of Drosophila synapsin, were identified in two separate families segregating epilepsy and in an ASD/epilepsy cohort (Garcia, Blair et al. 2004, Fassio, Patry et al. 2011). Drosophila Syxla is orthologous to human STX1B, and mutations in $S T X 1 B$ are associated with febrile seizures and epilepsy (Schubert, Siekierska et al. 2014). The data from our fly model suggests these proteins may be implicated in Dup15q epilepsy, and alterations in these synaptic proteins in the Dup15q brain remain to be explored.

Glial GST upregulation may broadly underlie treatment resistant epilepsies. GSTs are upregulated in the human brain in cases of treatment resistant epilepsy, particularly in astrocytes surrounding blood vessels (Shang, Liu et al. 2008). While the factor initiating

GST upregulation in seizure remains unknown, we show here that chemical induction of 
seizure activity did not result in GST up regulation, implying the neuronal activity alone is not the cause. GST upregulation occurred within glia in response to oxidative stress in a fly model of Alexander disease (Wang, Colodner et al. 2011, Wang, Hagemann et al. 2016) and in a mammalian in vitro culture system during increased neuronal activity (McGann and Mandel 2018). One hypothesis surrounding GSTs in treatment resistant epilepsy is glia of the blood brain barrier upregulate GSTs, whether from oxidative stress or increased neuronal activity during seizure, causing antiepileptic drug metabolism before they enter the brain. Indeed, in cancer GST upregulation is associated with increased drug metabolism and chemoresistance (Sau, Pellizzari Tregno et al. 2010, Allocati, Masulli et al. 2018), suggesting GST upregulation as a cause of treatment resistant epilepsy as well.

In conclusion, we found cell non-autonomous downregulation of synaptic transmission proteins in repo $>$ Dube $3 a$ flies combined with GST upregulation not only in Dup15q related repo $>$ Dube $3 a$ animals but across gliopathic epilepsies we tested. Our results highlight the use of Drosophila for investigating cell-type-specific genetic manipulations and provides novel avenues to investigate in Dup15q and treatment resistant epilepsy. 


\section{CHAPTER 6. DISCUSSION AND FUTURE DIRECTIONS}

\section{Imprinting Status of $U B E 3 A$ in Drosophila}

In 1970 reports of genomic imprinting came from observations that maize kernel coloring can be directly associated with the parent of origin of certain traits, rather than traditional dominant/recessive inheritance (Kermicle 1970). Genomic imprinting has since been observed in both flowering plants and placental mammals (Feil and Berger 2007), but not other lineages. $U B E 3 A$ demonstrates genomic imprinting in mature mammalian neurons where it is exclusively expressed from the maternal allele (Albrecht, Sutcliffe et al. 1997, Rougeulle, Glatt et al. 1997, LaSalle, Reiter et al. 2015). Despite a lack of evidence for any gene to be imprinted in insects, a report suggested imprinted or preferential expression of Dube3a in Drosophila (Chakraborty, Paul et al. 2015). In chapter 2 we present data demonstrating that Dube $3 a$ is not imprinted nor preferentially expressed from either allele in Drosophila (Hope, LeDoux et al. 2016). While the emergence of the $U B E 3 A$ gene is ancient, appearing after amoebozoa and before fungi, the emergence PWS-AS imprinting domain is not, and is found in only animal lineages after the opossum (Sato 2017). Our data indicating bi-allelic expression of Dube $3 a$ in Drosophila neurons is consistent with the current dogma that only flowering plants and placental mammals undergo genomic imprinting (Feil and Berger 2007).

\section{Interaction Between UBE3A and HERC2}

Until now, the interaction between UBE3A and HERC2 was predominantly studied using in vitro biochemical methods (Kuhnle, Kogel et al. 2011, Galligan, Martinez-Noel et al. 2015). Although in vitro evidence is clear that the RLD2 domain of HERC2 can directly interact with UBE3A causing an increase in UBE3A ubiquitin ligase activity, these interactions had not been shown to be occurring in vivo. In Chapter 3 we showed that the interaction between Drosophila Dube $3 a$ and HERC2 confirms the interaction observed with the mammalian homologs in vitro by increasing the ubiquitin ligase activity of Dube3a. This interaction also has measurable behavioral outcomes that appear to be the consequence of this interaction. We observed a synergistic interaction between Dube $3 a$ and $H E R C 2$ in social interaction and circadian rhythms where defects were only observed when both genes were overexpressed, implying that both proteins must interact for these phenotypes. These data suggest that genes located within the 15q11.2-q13.1 other than $U B E 3 A$ are contributing to Dup15q phenotypes and that these genes should not be overlooked when investigating Dup15q syndrome.

Recent work demonstrates that the binding of E6 to UBE3A acts as an allosteric modulator, altering the conformational shape of the UBE3A protein and through this steric change alters the ubiquitin ligase substrates identified by UBE3A (Sailer, Offensperger et al. 2018). Through these conformational changes, in the presence of E6 viral protein UBE3A targets p53 for degradation, while in the absence of E6, p53 is not a UBE3A substrate (Scheffner, Huibregtse et al. 1993). It is possible that when bound to 
HERC2 similar conformational changes occur, altering substrate recognition leading to the phenotypes we observed in our fly model that only emerged upon simultaneous overexpression of both proteins. Future studies should address whether HERC2, specifically the RLD2 domain that is known to bind UBE3A (Kuhnle, Kogel et al. 2011), alters the structural conformation of UBE3A and changes its substrate proteins in addition to increasing the ubiquitin ligase activity. Reducing the activity of UBE3A could be an effective and targeted treatment for Dup15q syndrome. The identification of individuals carrying $H E R C 2$ loss of function mutations mimicking AS suggests that HERC2 may be necessary for the normal function of UBE3A in vivo (Puffenberger, Jinks et al. 2012, Harlalka, Baple et al. 2013, Morice-Picard, Benard et al. 2016). Understanding this interaction could lead to the development of Dup15q therapeutics since compounds that disrupt the interaction between HERC2 and UBE3A may be an effective way to reduce UBE3A activity without the requirement of dramatically reducing UBE3A levels in Dup15q syndrome.

\section{A Possible Role for Glia in Dup15q Epilepsy}

Our fly model using glial overexpression of $U B E 3 A$ is the first animal model of Dup15q that recapitulates a clear seizure phenotype (Hope, LeDoux et al. 2017). We constructed a unified working model based on the data presented in Chapters 4 and 5 (Figure 6-1). The tripartite synapse consists of a presynaptic neuron, a postsynaptic neuron, and a glial cell (Perea, Navarrete et al. 2009). Upon elevation of UBE3A in glial cells we observed reduced levels of the $\mathrm{Na}^{+} / \mathrm{K}^{+}$ATPase, ATP $\alpha$, and reduced levels of intracellular $\mathrm{K}^{+}$within glial cells. We hypothesize that this leads to a buildup of $\mathrm{K}^{+}$in the extracellular space because $\mathrm{K}^{+}$is not being actively pumped into glial cells from the synaptic space. Elevated levels of $\mathrm{K}^{+}$surrounding neurons reduces the driving force for positively charged $\mathrm{K}^{+}$ions to leave the cell through $\mathrm{K}^{+}$leak channels, resulting in a depolarized resting membrane potential that is closer to action potential threshold (Figure 4-10). This could result in hyperexcitable neurons, as less excitatory input is needed to drive firing which tips networks into a hyperexcitable, seizure prone state. Proteomic and RNAseq studies we performed revealed reduced levels of synaptic signaling proteins predominantly located in the presynaptic compartment in a cell nonautonomous manner. The mechanism underlying the downregulation of these synaptic proteins remains to be explored, however it is possible that reduction in synaptic proteins is a compensatory mechanism to reduce the increased neuronal excitability caused by the elevated levels of extracellular $\mathrm{K}^{+}$. Finally, during our analysis we found a broad upregulation of GSTs cell autonomously within glia in response to Dube $3 a$ overexpression that is not dependent upon elevated neuronal activity alone. This upregulation of GSTs may partially explain the pharmacoresistance of Dup15q syndrome.

In the majority of the gliopathic seizure lines identified in Chapter 5 we observed an induction of GST gene expression. GstDl is regulated by the transcription factor $C n c$ and the repressor Keapl in Drosophila (NRF2 and KEAP1 in humans, respectively). Under oxidative stress conditions $C n c$ is relieved from Keapl repression and binds to 


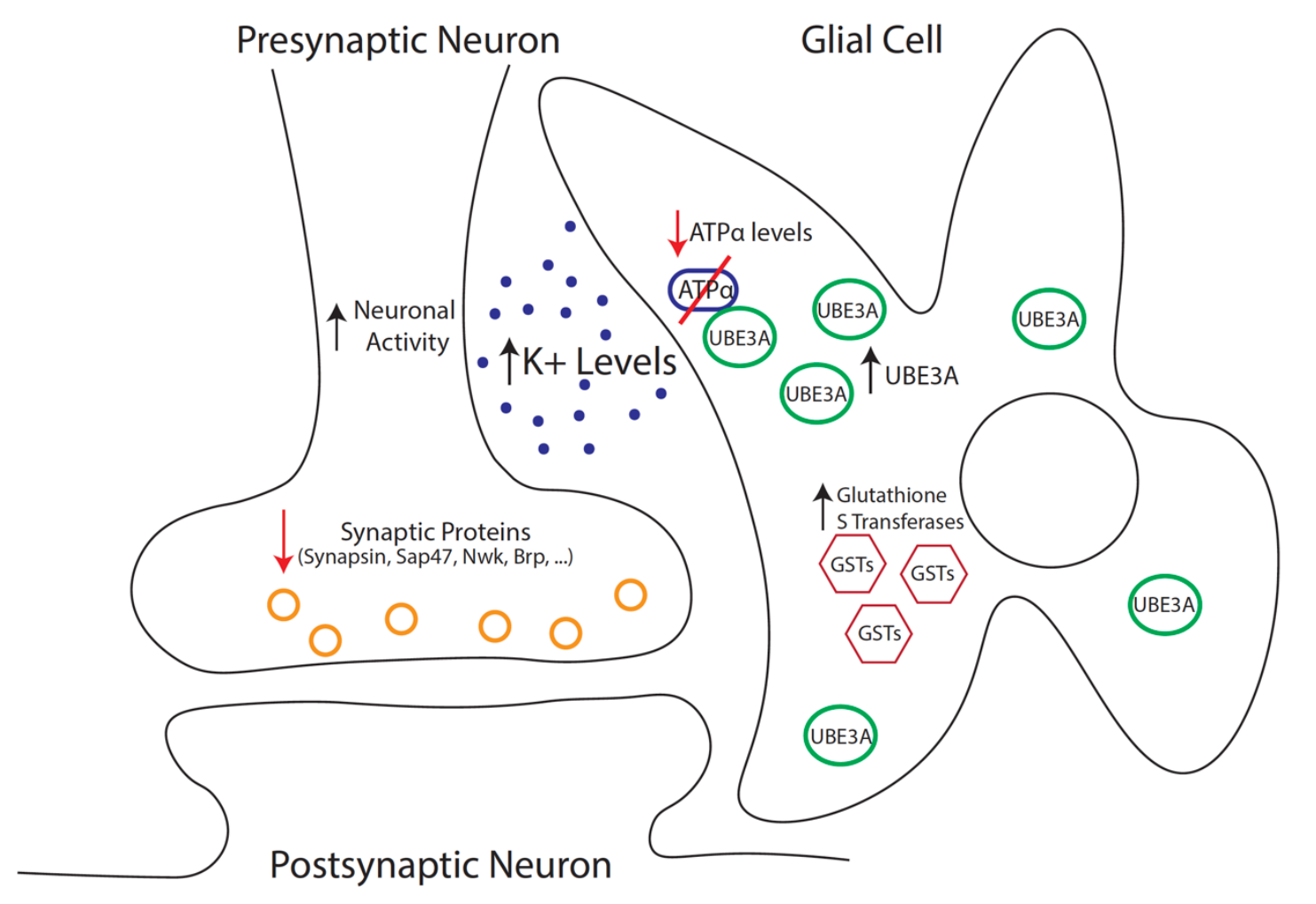

Figure 6-1. Unified working model of how elevated Dube3a in glia impacts the tripartite synapse.

In Chapter 4 we demonstrated that elevation of $D u b e 3 a$ in glia causes reduced levels of the $\mathrm{Na}^{+} / \mathrm{K}^{+}$ATPase ATP $\alpha$ leading to a buildup of $\mathrm{K}^{+}$in the extracellular space, and increased extracellular $\mathrm{K}^{+}$causes increased neuronal activity, leading to seizure-like activity. In Chapter 5 we found a cell non-autonomous downregulation of synaptic proteins in neurons. We also observed an upregulation of GSTs cell autonomously within glial cells. One or more of these changes may be responsible for the seizure susceptibility of repo $>$ Dube $3 a$ flies. 
antioxidant response elements throughout the genome to induce oxidative stress response genes, including GstD1 (Sykiotis and Bohmann 2008). Future experiments should investigate whether glial cells are undergoing a generalized oxidative stress response, as is suggested by the upregulation of GstD1, and whether this is the cause of seizures or a secondary response.

Data presented in Chapter 5 suggests that glial cell dysfunction itself may underlie epileptic pathologies other than Dup15q syndrome. We observed seizures in flies with knockdown of both the $\alpha$ and $\beta$ subunits of the $\mathrm{Na}^{+} / \mathrm{K}^{+}$pump. Potassium dysregulation in glia has been proposed as a mechanism that underlies seizure biogenesis before (Devinsky, Vezzani et al. 2013). Additionally, glial-specific knockdown of the mitochondrial related genes $C O X 7 A$ or $\operatorname{Ses} B$ resulted in seizure phenotypes, as did glialspecific knockdown of comatose (comt), the gene encoding N-ethylmaleimide-sensitive factor 1. Mitochondrial dysfunction has also been proposed as a cause of epilepsy (Khurana, Valencia et al. 2013), and vesicle release machinery and exocytosis occurs in astrocytes (reviewed in Vardjan, Parpura et al. 2016). Our findings suggest that altering mitochondrial or vesicle function in glia results in seizure susceptibility and causes the induction of GSTs.

In these experiments we used the pan-glial driver repo-GAL4 to drive expression of constructs specifically within glial cells. However, Drosophila have multiple glial subtypes within the central nervous system including astrocyte-like glia that are functionally similar to mammalian astrocytes, cortex glia that are intimately associated with neuronal cell bodies, and ensheathing glia that partition off portions of the fly brain into discrete compartments (Freeman 2015). Preliminary data from our lab suggests that elevated levels of Dube $3 a$ in astrocyte-like and cortex glia in flies contributes to the seizure phenotype. Indeed, knockdown of the $\mathrm{Na}^{+} / \mathrm{Ca}^{2+}$ exchanger zydeco in Drosophila cortex glia resulted in seizure and hyperexcitability (Melom and Littleton 2013) as did the cation-chloride cotransporter kazachoc (Rusan, Kingsford et al. 2014). Multiple proteins expressed within astrocyte-like glia critically regulate neuronal excitability (Cho, Muthukumar et al. 2018). Further studies should establish the specific glial subtypes responsible for seizure production in each gliopathic seizure line, as this may provide insight into the mechanism of glial driven seizures.

\section{Insights into Treatment Strategies}

Data presented in Chapter 4 indicates that overexpression of Dube $3 a$ in glia prior to the adult stage results in seizure susceptibility, however limiting overexpression of Dube $3 a$ in glia during adulthood was not sufficient to cause seizures (Figure 4-5). These data are consistent with findings in AS mouse models that indicate reinstating Ube $3 a$ in neurons in adulthood does not rescue phenotypes observed in AS mice (Silva-Santos, van Woerden et al. 2015). These data suggest some fixed change occurs in the brain during early development caused by too little or too much UBE3A that results in long lasting effects on behavior, including seizure susceptibility, which are not able to be rescued by normalizing UBE3A levels. The earlier during development that a treatment strategy can 
be implemented, the better the likelihood it will be effective in controlling Dup15q seizures.

The finding that GSTs are upregulated in several of our gliopathic seizure fly lines is significant since upregulation of GSTs occurs in cases of idiopathic treatment resistant epilepsy in astrocytes surrounding blood vessels and may contribute to the treatment resistant nature of the seizures (Shang, Liu et al. 2008). GST enzymes conjugate glutathione to small molecules facilitating their metabolism. GST upregulation is hypothesized to underlie anti-cancer drug resistance in some instances, and may contribute to the metabolism of AEDs (Townsend and Tew 2003, Allocati, Masulli et al. 2018). We are currently investigating whether GST upregulation occurs in the human brain in Dup15q syndrome. If GSTs are elevated in astrocytes, as is predicted by our Drosophila model, particularly in astrocytes surrounding blood vessels, as has been observed in idiopathic treatment resistant epilepsies (Shang, Liu et al. 2008), then it may be necessary to use a combination therapy approach which combines an AED with a GST inhibitor, such as ethacrynic acid or Terrapin 199 (Tew, Dutta et al. 1997), to reduce metabolism of the AED.

All of the experiments performed in this work were conducted in the model organism Drosophila melanogaster. Both Drosophila Dube $3 a$ and human UBE3A overexpression in fly glia causes a seizure phenotype, suggesting that this E3 ubiquitin ligase can cause seizures when elevated in glia in humans as well. However, further research needs to be conducted to determine whether glial $U B E 3 A$ overexpression in mammalian glia also causes seizures, and whether glial $U B E 3 A$ overexpression ultimately causes seizures in Dup15q syndrome. Mammalian glial cells can be broadly broken down into three classes consisting of astrocytes, oligodendrocytes, and microglia (Jakel and Dimou 2017). Drosophila astrocyte-like glia and cortex glia appear to be the primary glial subtypes that underlie Drosophila gliopathic seizures, and these glia are also the most similar in structure and function to mammalian astrocytes (Freeman 2015). Future work will determine if overexpression of $U B E 3 A$ in mouse astrocytes generates seizures and if the molecular changes observed in our fly model of Dup15q epilepsy, such as GST upregulation, synaptic protein downregulation, and elevated levels of $U B E 3 A$ in astrocytes occur in the Dup $15 \mathrm{q}$ syndrome.

In summary, we demonstrated that Dube $3 a$ is not imprinted in the fly. We also found that Dube3a and HERC2 interact synergistically to cause ASD-like phenotypes in Drosophila that are similar to phenotypes observed in Dup15q. These data show that Dup15q should be studied more holistically and genes located in the 15q11.2-q13.1 region other that $U B E 3 A$ should not be ignored. Finally, during the course of my thesis work we constructed the first model of Dup $15 \mathrm{q}$ that recapitulates the seizure phenotype of Dup 15q syndrome. Our fly model suggests, for the first time, that glial cells may underlie Dup15q seizures. Efforts are now underway to translate our findings from the fly to mammalian systems. 


\section{LIST OF REFERENCES}

Al Ageeli, E., S. Drunat, C. Delanoe, L. Perrin, C. Baumann, Y. Capri, J. Fabre-Teste, A. Aboura, C. Dupont, S. Auvin, L. El Khattabi, D. Chantereau, A. Moncla, A. C. Tabet and A. Verloes (2014). "Duplication of the 15q11-q13 region: clinical and genetic study of 30 new cases." Eur J Med Genet 57(1): 5-14.

Al-Hakim, A. K., M. Bashkurov, A. C. Gingras, D. Durocher and L. Pelletier (2012). "Interaction proteomics identify NEURL4 and the HECT E3 ligase HERC2 as novel modulators of centrosome architecture." Mol Cell Proteomics 11(6): M111 014233.

Albrecht, U., J. S. Sutcliffe, B. M. Cattanach, C. V. Beechey, D. Armstrong, G. Eichele and A. L. Beaudet (1997). "Imprinted expression of the murine Angelman syndrome gene, Ube3a, in hippocampal and Purkinje neurons." Nat Genet 17(1): 75-78.

Allocati, N., M. Masulli, C. Di Ilio and L. Federici (2018). "Glutathione transferases: substrates, inihibitors and pro-drugs in cancer and neurodegenerative diseases." Oncogenesis 7(1): 8.

American Psychiatric Association (2013). Diagnostic and Statistical Manual of Mental Disorders: DSM-5.5th edn. Arlington, Virginia, USA.

Aradska, J., T. Bulat, F. J. Sialana, R. Birner-Gruenberger, B. Erich and G. Lubec (2015). "Gel-free mass spectrometry analysis of Drosophila melanogaster heads." Proteomics 15(19): 3356-3360.

Bailey, J. A., Z. Gu, R. A. Clark, K. Reinert, R. V. Samonte, S. Schwartz, M. D. Adams, E. W. Myers, P. W. Li and E. E. Eichler (2002). "Recent segmental duplications in the human genome." Science 297(5583): 1003-1007.

Baio, J., L. Wiggins, D. L. Christensen, M. J. Maenner, J. Daniels, Z. Warren, M. Kurzius-Spencer, W. Zahorodny, C. Robinson Rosenberg, T. White, M. S. Durkin, P. Imm, L. Nikolaou, M. Yeargin-Allsopp, L. C. Lee, R. Harrington, M. Lopez, R. T. Fitzgerald, A. Hewitt, S. Pettygrove, J. N. Constantino, A. Vehorn, J. Shenouda, J. HallLande, K. Van Naarden Braun and N. F. Dowling (2018). "Prevalence of Autism Spectrum Disorder Among Children Aged 8 Years - Autism and Developmental Disabilities Monitoring Network, 11 Sites, United States, 2014." MMWR Surveill Summ 67(6): 1-23.

Battaglia, A. (2008). "The inv dup (15) or idic (15) syndrome (Tetrasomy 15q)." Orphanet J Rare Dis 3: 30.

Bekker-Jensen, S., J. Rendtlew Danielsen, K. Fugger, I. Gromova, A. Nerstedt, C. Lukas, J. Bartek, J. Lukas and N. Mailand (2010). "HERC2 coordinates ubiquitin-dependent assembly of DNA repair factors on damaged chromosomes." Nat Cell Biol 12(1): 80-86; sup pp 81-12. 
Benzer, S. (1971). "From the gene to behavior." JAMA 218(7): 1015-1022.

Bhatt, R., A. Dickinson, C. Hyde, S. Rallipalli, K. Dahlerbruch, C. Rocha and S. Jeste (2018). "Quantitative Measures of Motor Function in children with Duplications of 15q11.3-13.1 (Dup15q Syndrome) and Typically Developing (TD) Children." Neurology 90.

Bischof, J., M. Bjorklund, E. Furger, C. Schertel, J. Taipale and K. Basler (2013). "A versatile platform for creating a comprehensive UAS-ORFeome library in Drosophila." Development 140(11): 2434-2442.

Boronat, S., W. A. Mehan, E. A. Shaaya, R. L. Thibert and P. Caruso (2015). "Hippocampal abnormalities in magnetic resonance imaging (MRI) of $15 \mathrm{q}$ duplication syndromes." J Child Neurol 30(3): 333-338.

Brand, A. H. and N. Perrimon (1993). "Targeted gene expression as a means of altering cell fates and generating dominant phenotypes." Development 118(2): 401-415.

Buiting, K., C. Williams and B. Horsthemke (2016). "Angelman syndrome - insights into a rare neurogenetic disorder." Nat Rev Neurol 12(10): 584-593.

Burette, A. C., M. C. Judson, A. N. Li, E. F. Chang, W. W. Seeley, B. D. Philpot and R. J. Weinberg (2018). "Subcellular organization of UBE3A in human cerebral cortex." $\underline{\text { Mol }}$ Autism 9: 54.

Butler, K. M., O. A. Moody, E. Schuler, J. Coryell, J. J. Alexander, A. Jenkins and A. Escayg (2018). "De novo variants in GABRA2 and GABRA5 alter receptor function and contribute to early-onset epilepsy." Brain.

Chakraborty, M., B. K. Paul, T. Nayak, A. Das, N. R. Jana and S. Bhutani (2015). "The E3 ligase ube3a is required for learning in Drosophila melanogaster." Biochem Biophys Res Commun 462(1): 71-77.

Chamberlain, S. J., P. F. Chen, K. Y. Ng, F. Bourgois-Rocha, F. Lemtiri-Chlieh, E. S. Levine and M. Lalande (2010). "Induced pluripotent stem cell models of the genomic imprinting disorders Angelman and Prader-Willi syndromes." Proc Natl Acad Sci U S A 107(41): 17668-17673.

Chan, N. C., W. den Besten, M. J. Sweredoski, S. Hess, R. J. Deshaies and D. C. Chan (2014). "Degradation of the deubiquitinating enzyme USP33 is mediated by p97 and the ubiquitin ligase HERC2." J Biol Chem 289(28): 19789-19798.

Cho, S., A. K. Muthukumar, T. Stork, J. C. Coutinho-Budd and M. R. Freeman (2018). "Focal adhesion molecules regulate astrocyte morphology and glutamate transporters to suppress seizure-like behavior." Proc Natl Acad Sci U S A 115(44): 11316-11321.

Chvatal, A. and E. Sykova (2000). "Glial influence on neuronal signaling." Prog Brain Res 125: 199-216. 
Clayton-Smith, J., T. Webb, X. J. Cheng, M. E. Pembrey and S. Malcolm (1993). "Duplication of chromosome 15 in the region 15q11-13 in a patient with developmental delay and ataxia with similarities to Angelman syndrome." J Med Genet 30(6): 529-531.

Conant, K. D., B. Finucane, N. Cleary, A. Martin, C. Muss, M. Delany, E. K. Murphy, O. Rabe, K. Luchsinger, S. J. Spence, C. Schanen, O. Devinsky, E. H. Cook, J. LaSalle, L. T. Reiter and R. L. Thibert (2014). "A survey of seizures and current treatments in 15q duplication syndrome." Epilepsia 55(3): 396-402.

Cook, E. H., Jr., V. Lindgren, B. L. Leventhal, R. Courchesne, A. Lincoln, C. Shulman, C. Lord and E. Courchesne (1997). "Autism or atypical autism in maternally but not paternally derived proximal 15q duplication." Am J Hum Genet 60(4): 928-934.

Cooper, E. M., A. W. Hudson, J. Amos, J. Wagstaff and P. M. Howley (2004). "Biochemical analysis of Angelman syndrome-associated mutations in the E3 ubiquitin ligase E6-associated protein." J Biol Chem 279(39): 41208-41217.

Copping, N., S. Christian, D. Ritter, M. Islam, N. Buscher, D. Zolkowska, M. Pride, E. Berg, J. LaSalle, J. Ellegood, J. Lerch, L. T. Reiter, J. Silverman and S. Dindot (2017). "Neuronal overexpression of Ube3a isoform 2 causes behavioral impairments and neuroanatomical pathology relevant to 15q11.2-q13.3 duplication syndrome." Human Molecular Genetics.

Coulter, D. A. and C. Steinhauser (2015). "Role of astrocytes in epilepsy." Cold Spring Harb Perspect Med 5(3): a022434.

Cubillos-Rojas, M., T. Schneider, O. Hadjebi, L. Pedrazza, J. R. de Oliveira, F. Langa, J. L. Guenet, J. Duran, J. M. de Anta, S. Alcantara, R. Ruiz, E. M. Perez-Villegas, F. J. Aguilar-Montilla, A. M. Carrion, J. A. Armengol, E. Baple, A. H. Crosby, R. Bartrons, F. Ventura and J. L. Rosa (2016). "The HERC2 ubiquitin ligase is essential for embryonic development and regulates motor coordination." Oncotarget 7(35): 56083-56106.

D'Ambrosio, R. (2004). "The role of glial membrane ion channels in seizures and epileptogenesis." Pharmacol Ther 103(2): 95-108.

De Fusco, M., R. Marconi, L. Silvestri, L. Atorino, L. Rampoldi, L. Morgante, A. Ballabio, P. Aridon and G. Casari (2003). "Haploinsufficiency of ATP1A2 encoding the $\mathrm{Na}+/ \mathrm{K}+$ pump alpha2 subunit associated with familial hemiplegic migraine type $2 . "$ Nat Genet 33(2): 192-196.

Depienne, C., D. Moreno-De-Luca, D. Heron, D. Bouteiller, A. Gennetier, R. Delorme, P. Chaste, J. P. Siffroi, S. Chantot-Bastaraud, B. Benyahia, O. Trouillard, G. Nygren, S. Kopp, M. Johansson, M. Rastam, L. Burglen, E. Leguern, A. Verloes, M. Leboyer, A. Brice, C. Gillberg and C. Betancur (2009). "Screening for genomic rearrangements and methylation abnormalities of the 15q11-q13 region in autism spectrum disorders." Biol Psychiatry 66(4): 349-359. 
Deprez, L., S. Weckhuysen, K. Peeters, T. Deconinck, K. G. Claeys, L. R. Claes, A. Suls, T. Van Dyck, A. Palmini, G. Matthijs, W. Van Paesschen and P. De Jonghe (2008). "Epilepsy as part of the phenotype associated with ATP1A2 mutations." Epilepsia 49(3): 500-508.

Desai-Shah, M. and R. L. Cooper (2009). "Different mechanisms of Ca2+ regulation that influence synaptic transmission: comparison between crayfish and Drosophila neuromuscular junctions." Synapse 63(12): 1100-1121.

Devinsky, O., A. Vezzani, S. Najjar, N. C. De Lanerolle and M. A. Rogawski (2013). "Glia and epilepsy: excitability and inflammation." Trends Neurosci 36(3): 174-184.

Dindot, S. V., B. A. Antalffy, M. B. Bhattacharjee and A. L. Beaudet (2008). "The Angelman syndrome ubiquitin ligase localizes to the synapse and nucleus, and maternal deficiency results in abnormal dendritic spine morphology." Hum Mol Genet 17(1): 111118.

DiStefano, C., A. Gulsrud, S. Huberty, C. Kasari, E. Cook, L. T. Reiter, R. Thibert and S. S. Jeste (2016). "Identification of a distinct developmental and behavioral profile in children with Dup15q syndrome." J Neurodev Disord 8: 19.

Dittrich, B., K. Buiting, B. Korn, S. Rickard, J. Buxton, S. Saitoh, R. D. Nicholls, A. Poustka, A. Winterpacht, B. Zabel and B. Horsthemke (1996). "Imprint switching on human chromosome 15 may involve alternative transcripts of the SNRPN gene." Nat Genet 14(2): 163-170.

Duffy, J. B. (2002). "GAL4 system in Drosophila: a fly geneticist's Swiss army knife." Genesis 34(1-2): 1-15.

Edwards, T. N. and I. A. Meinertzhagen (2010). "The functional organisation of glia in the adult brain of Drosophila and other insects." Prog Neurobiol 90(4): 471-497.

Fassio, A., L. Patry, S. Congia, F. Onofri, A. Piton, J. Gauthier, D. Pozzi, M. Messa, E. Defranchi, M. Fadda, A. Corradi, P. Baldelli, L. Lapointe, J. St-Onge, C. Meloche, L. Mottron, F. Valtorta, D. Khoa Nguyen, G. A. Rouleau, F. Benfenati and P. Cossette (2011). "SYN1 loss-of-function mutations in autism and partial epilepsy cause impaired synaptic function." Hum Mol Genet 20(12): 2297-2307.

Feil, R. and F. Berger (2007). "Convergent evolution of genomic imprinting in plants and mammals." Trends Genet 23(4): 192-199.

Ferdousy, F., W. Bodeen, K. Summers, O. Doherty, O. Wright, N. Elsisi, G. Hilliard, J. M. O'Donnell and L. T. Reiter (2011). "Drosophila Ube3a regulates monoamine synthesis by increasing GTP cyclohydrolase I activity via a non-ubiquitin ligase mechanism." Neurobiol Dis 41(3): 669-677. 
Fiest, K. M., K. M. Sauro, S. Wiebe, S. B. Patten, C. S. Kwon, J. Dykeman, T.

Pringsheim, D. L. Lorenzetti and N. Jette (2017). "Prevalence and incidence of epilepsy: A systematic review and meta-analysis of international studies." Neurology 88(3): 296303.

Finley, D. (2009). "Recognition and processing of ubiquitin-protein conjugates by the proteasome." Annu Rev Biochem 78: 477-513.

Finucane, B. M., L. Lusk, D. Arkilo, S. Chamberlain, O. Devinsky, S. Dindot, S. S. Jeste, J. M. LaSalle, L. T. Reiter, N. C. Schanen, S. J. Spence, R. L. Thibert, G. Calvert, K. Luchsinger and E. H. Cook (2016). 15q Duplication Syndrome and Related Disorders. GeneReviews(R). R. A. Pagon, M. P. Adam, H. H. Ardinger et al. Seattle (WA).

Freeman, M. R. (2015). "Drosophila Central Nervous System Glia." Cold Spring Harb Perspect Biol 7(11).

Frohlich, J., M. T. Miller, L. M. Bird, P. Garces, H. Purtell, M. C. Hoener, B. D. Philpot, M. S. Sidorov, W. H. Tan, M. C. Hernandez, A. Rotenberg, S. S. Jeste, M. Krishnan, O. Khwaja and J. F. Hipp (2019). "Electrophysiological Phenotype in Angelman Syndrome Differs Between Genotypes." Biol Psychiatry.

Frohlich, J., D. Senturk, V. Saravanapandian, P. Golshani, L. T. Reiter, R. Sankar, R. L. Thibert, C. DiStefano, S. Huberty, E. H. Cook and S. S. Jeste (2016). "A Quantitative Electrophysiological Biomarker of Duplication 15q11.2-q13.1 Syndrome." PLoS One 11(12): e0167179.

Gallanti, A., A. Tonelli, V. Cardin, G. Bussone, N. Bresolin and M. T. Bassi (2008). "A novel de novo nonsense mutation in ATP1A2 associated with sporadic hemiplegic migraine and epileptic seizures." J Neurol Sci 273(1-2): 123-126.

Galligan, J. T., G. Martinez-Noel, V. Arndt, S. Hayes, T. W. Chittenden, J. W. Harper and P. M. Howley (2015). "Proteomic analysis and identification of cellular interactors of the giant ubiquitin ligase HERC2." J Proteome Res 14(2): 953-966.

Ganetzky, B. and C. F. Wu (1982). "Drosophila mutants with opposing effects on nerve excitability: genetic and spatial interactions in repetitive firing." J Neurophysiol 47(3): 501-514.

Garcia, C. C., H. J. Blair, M. Seager, A. Coulthard, S. Tennant, M. Buddles, A. Curtis and J. A. Goodship (2004). "Identification of a mutation in synapsin I, a synaptic vesicle protein, in a family with epilepsy." J Med Genet 41(3): 183-186.

Golyala, A. and P. Kwan (2017). "Drug development for refractory epilepsy: The past 25 years and beyond." Seizure 44: 147-156.

Gorska-Andrzejak, J. (2013). "Glia-related circadian plasticity in the visual system of Diptera." Front Physiol 4: 36. 
Gossan, N. C., F. Zhang, B. Guo, D. Jin, H. Yoshitane, A. Yao, N. Glossop, Y. Q. Zhang, Y. Fukada and Q. J. Meng (2014). "The E3 ubiquitin ligase UBE3A is an integral component of the molecular circadian clock through regulating the BMAL1 transcription factor." Nucleic Acids Res 42(9): 5765-5775.

Grice, S. J., J. L. Liu and C. Webber (2015). "Synergistic interactions between Drosophila orthologues of genes spanned by de novo human CNVs support multiple-hit models of autism." PLoS Genet 11(3): e1004998.

Grier, M. D., R. P. Carson and A. H. Lagrange (2015). "Toward a Broader View of Ube3a in a Mouse Model of Angelman Syndrome: Expression in Brain, Spinal Cord, Sciatic Nerve and Glial Cells." PLoS One 10(4): e0124649.

Gu, W., F. Zhang and J. R. Lupski (2008). "Mechanisms for human genomic rearrangements." Pathogenetics 1(1): 4.

Guo, H., T. Wang, H. Wu, M. Long, B. P. Coe, H. Li, G. Xun, J. Ou, B. Chen, G. Duan, T. Bai, N. Zhao, Y. Shen, Y. Li, Y. Wang, Y. Zhang, C. Baker, Y. Liu, N. Pang, L. Huang, L. Han, X. Jia, C. Liu, H. Ni, X. Yang, L. Xia, J. Chen, L. Shen, Y. Li, R. Zhao, W. Zhao, J. Peng, Q. Pan, Z. Long, W. Su, J. Tan, X. Du, X. Ke, M. Yao, Z. Hu, X. Zou, J. Zhao, R. A. Bernier, E. E. Eichler and K. Xia (2018). "Inherited and multiple de novo mutations in autism/developmental delay risk genes suggest a multifactorial model." $\underline{\mathrm{Mol}}$ Autism 9: 64.

Harlalka, G. V., E. L. Baple, H. Cross, S. Kuhnle, M. Cubillos-Rojas, K. Matentzoglu, M. A. Patton, K. Wagner, R. Coblentz, D. L. Ford, D. J. Mackay, B. A. Chioza, M. Scheffner, J. L. Rosa and A. H. Crosby (2013). "Mutation of HERC2 causes developmental delay with Angelman-like features." J Med Genet 50(2): 65-73.

Hatfield, I., I. Harvey, E. R. Yates, J. R. Redd, L. T. Reiter and D. Bridges (2015). "The role of TORC1 in muscle development in Drosophila." Sci Rep 5: 9676.

Hogart, A., K. N. Leung, N. J. Wang, D. J. Wu, J. Driscoll, R. O. Vallero, N. C. Schanen and J. M. LaSalle (2009). "Chromosome 15q11-13 duplication syndrome brain reveals epigenetic alterations in gene expression not predicted from copy number." J Med Genet 46(2): 86-93.

Hogart, A., D. Wu, J. M. LaSalle and N. C. Schanen (2010). "The comorbidity of autism with the genomic disorders of chromosome 15q11.2-q13." Neurobiol Dis 38(2): 181-191.

Hope, K. A., M. S. LeDoux and L. T. Reiter (2016). "The Drosophila melanogaster homolog of UBE3A is not imprinted in neurons." Epigenetics 11(9): 637-642.

Hope, K. A., M. S. LeDoux and L. T. Reiter (2017). "Glial overexpression of Dube3a causes seizures and synaptic impairments in Drosophila concomitant with down regulation of the $\mathrm{Na}+/ \mathrm{K}+$ pump ATPalpha." Neurobiol Dis 108: 238-248. 
Huang da, W., B. T. Sherman and R. A. Lempicki (2009). "Bioinformatics enrichment tools: paths toward the comprehensive functional analysis of large gene lists." Nucleic Acids Res 37(1): 1-13.

Huang da, W., B. T. Sherman and R. A. Lempicki (2009). "Systematic and integrative analysis of large gene lists using DAVID bioinformatics resources." Nat Protoc 4(1): 4457.

Huibregtse, J. M., M. Scheffner and P. M. Howley (1993). "Cloning and expression of the cDNA for E6-AP, a protein that mediates the interaction of the human papillomavirus E6 oncoprotein with p53." Mol Cell Biol 13(2): 775-784.

Hummel, T., K. Krukkert, J. Roos, G. Davis and C. Klambt (2000). "Drosophila Futsch/22C10 is a MAP1B-like protein required for dendritic and axonal development." Neuron 26(2): 357-370.

Jakel, S. and L. Dimou (2017). "Glial Cells and Their Function in the Adult Brain: A Journey through the History of Their Ablation." Front Cell Neurosci 11: 24.

Jensen, L., M. F. Farook and L. T. Reiter (2013). "Proteomic profiling in Drosophila reveals potential Dube3a regulation of the actin cytoskeleton and neuronal homeostasis." PLoS One 8(4): e61952.

Jiang, X., R. Bomgarden, J. Brown, D. L. Drew, A. M. Robitaille, R. Viner and A. R. Huhmer (2017). "Sensitive and Accurate Quantitation of Phosphopeptides Using TMT Isobaric Labeling Technique." J Proteome Res 16(11): 4244-4252.

Judson, M. C., J. O. Sosa-Pagan, W. A. Del Cid, J. E. Han and B. D. Philpot (2014). "Allelic specificity of Ube3a expression in the mouse brain during postnatal development." J Comp Neurol 522(8): 1874-1896.

Jurkat-Rott, K., T. Freilinger, J. P. Dreier, J. Herzog, H. Gobel, G. C. Petzold, P. Montagna, T. Gasser, F. Lehmann-Horn and M. Dichgans (2004). "Variability of familial hemiplegic migraine with novel A1A2 Na+/K+-ATPase variants." Neurology 62(10): 1857-1861.

Kelsey, G. and R. Feil (2013). "New insights into establishment and maintenance of DNA methylation imprints in mammals." Philosophical Transactions of the Royal Society B-Biological Sciences 368(1609).

Kent, W. J., C. W. Sugnet, T. S. Furey, K. M. Roskin, T. H. Pringle, A. M. Zahler and D. Haussler (2002). "The human genome browser at UCSC." Genome Res 12(6): 996-1006.

Kermicle, J. L. (1970). "Dependence of the R-mottled aleurone phenotype in maize on mode of sexual transmission." Genetics 66(1): 69-85.

Khurana, D. S., I. Valencia, M. J. Goldenthal and A. Legido (2013). "Mitochondrial dysfunction in epilepsy." Semin Pediatr Neurol 20(3): 176-187. 
Kim, H. C. and J. M. Huibregtse (2009). "Polyubiquitination by HECT E3s and the determinants of chain type specificity." Mol Cell Biol 29(12): 3307-3318.

Kishino, T., M. Lalande and J. Wagstaff (1997). "UBE3A/E6-AP mutations cause Angelman syndrome." Nat Genet 15(1): 70-73.

Knoll, J. H., R. D. Nicholls, R. E. Magenis, J. M. Graham, Jr., M. Lalande and S. A. Latt (1989). "Angelman and Prader-Willi syndromes share a common chromosome 15 deletion but differ in parental origin of the deletion." Am J Med Genet 32(2): 285-290.

Krishnan, V., D. C. Stoppel, Y. Nong, M. A. Johnson, M. J. Nadler, E. Ozkaynak, B. L. Teng, I. Nagakura, F. Mohammad, M. A. Silva, S. Peterson, T. J. Cruz, E. M. Kasper, R. Arnaout and M. P. Anderson (2017). "Autism gene Ube3a and seizures impair sociability by repressing VTA Cbln1." Nature 543(7646): 507-512.

Kuhnle, S., U. Kogel, S. Glockzin, A. Marquardt, A. Ciechanover, K. Matentzoglu and M. Scheffner (2011). "Physical and functional interaction of the HECT ubiquitin-protein ligases E6AP and HERC2." J Biol Chem 286(22): 19410-19416.

Kwan, P. and M. J. Brodie (2000). "Early identification of refractory epilepsy." N Engl J Med 342(5): 314-319.

LaPenna, P. and L. M. Tormoehlen (2017). "The Pharmacology and Toxicology of ThirdGeneration Anticonvulsant Drugs." J Med Toxicol 13(4): 329-342.

LaSalle, J. M., L. T. Reiter and S. J. Chamberlain (2015). "Epigenetic regulation of UBE3A and roles in human neurodevelopmental disorders." Epigenomics 7(7): 12131228.

Lee, S. Y., J. Ramirez, M. Franco, B. Lectez, M. Gonzalez, R. Barrio and U. Mayor (2014). "Ube3a, the E3 ubiquitin ligase causing Angelman syndrome and linked to autism, regulates protein homeostasis through the proteasomal shuttle Rpn10." Cell Mol Life Sci 71(14): 2747-2758.

Lehman, A. L., Y. Nakatsu, A. Ching, R. T. Bronson, R. J. Oakey, N. Keiper-Hrynko, J. N. Finger, D. Durham-Pierre, D. B. Horton, J. M. Newton, M. F. Lyon and M. H. Brilliant (1998). "A very large protein with diverse functional motifs is deficient in rjs (runty, jerky, sterile) mice." Proc Natl Acad Sci U S A 95(16): 9436-9441.

Lewis, M. W., J. O. Brant, J. M. Kramer, J. I. Moss, T. P. Yang, P. J. Hansen, R. S. Williams and J. L. Resnick (2015). "Angelman syndrome imprinting center encodes a transcriptional promoter." Proc Natl Acad Sci U S A 112(22): 6871-6875.

Lu, Y., F. Wang, Y. Li, J. Ferris, J. A. Lee and F. B. Gao (2009). "The Drosophila homologue of the Angelman syndrome ubiquitin ligase regulates the formation of terminal dendritic branches." Hum Mol Genet 18(3): 454-462. 
Mackay, T. F., S. Richards, E. A. Stone, A. Barbadilla, J. F. Ayroles, D. Zhu, S. Casillas, Y. Han, M. M. Magwire, J. M. Cridland, M. F. Richardson, R. R. Anholt, M. Barron, C. Bess, K. P. Blankenburg, M. A. Carbone, D. Castellano, L. Chaboub, L. Duncan, Z. Harris, M. Javaid, J. C. Jayaseelan, S. N. Jhangiani, K. W. Jordan, F. Lara, F. Lawrence, S. L. Lee, P. Librado, R. S. Linheiro, R. F. Lyman, A. J. Mackey, M. Munidasa, D. M. Muzny, L. Nazareth, I. Newsham, L. Perales, L. L. Pu, C. Qu, M. Ramia, J. G. Reid, S. M. Rollmann, J. Rozas, N. Saada, L. Turlapati, K. C. Worley, Y. Q. Wu, A. Yamamoto, Y. Zhu, C. M. Bergman, K. R. Thornton, D. Mittelman and R. A. Gibbs (2012). "The Drosophila melanogaster Genetic Reference Panel." Nature 482(7384): 173-178.

Malhotra, D. and J. Sebat (2012). "CNVs: harbingers of a rare variant revolution in psychiatric genetics." Cell 148(6): 1223-1241.

Marshall, C. R., A. Noor, J. B. Vincent, A. C. Lionel, L. Feuk, J. Skaug, M. Shago, R. Moessner, D. Pinto, Y. Ren, B. Thiruvahindrapduram, A. Fiebig, S. Schreiber, J. Friedman, C. E. Ketelaars, Y. J. Vos, C. Ficicioglu, S. Kirkpatrick, R. Nicolson, L. Sloman, A. Summers, C. A. Gibbons, A. Teebi, D. Chitayat, R. Weksberg, A. Thompson, C. Vardy, V. Crosbie, S. Luscombe, R. Baatjes, L. Zwaigenbaum, W. Roberts, B. Fernandez, P. Szatmari and S. W. Scherer (2008). "Structural variation of chromosomes in autism spectrum disorder." Am J Hum Genet 82(2): 477-488.

McGann, J. C. and G. Mandel (2018). "Neuronal activity induces glutathione metabolism gene expression in astrocytes." Glia 66(9): 2024-2039.

McGrail, K. M., J. M. Phillips and K. J. Sweadner (1991). "Immunofluorescent localization of three $\mathrm{Na}, \mathrm{K}-\mathrm{ATPase}$ isozymes in the rat central nervous system: both neurons and glia can express more than one Na,K-ATPase." J Neurosci 11(2): 381-391.

Melom, J. E. and J. T. Littleton (2013). "Mutation of a NCKX eliminates glial microdomain calcium oscillations and enhances seizure susceptibility." J Neurosci 33(3): 1169-1178.

Minassian, B. A., T. M. DeLorey, R. W. Olsen, M. Philippart, Y. Bronstein, Q. Zhang, R. Guerrini, P. Van Ness, M. O. Livet and A. V. Delgado-Escueta (1998). "Angelman syndrome: correlations between epilepsy phenotypes and genotypes." Ann Neurol 43(4): 485-493.

Moreno-De-Luca, D., S. J. Sanders, A. J. Willsey, J. G. Mulle, J. K. Lowe, D. H. Geschwind, M. W. State, C. L. Martin and D. H. Ledbetter (2013). "Using large clinical data sets to infer pathogenicity for rare copy number variants in autism cohorts." Mol Psychiatry 18(10): 1090-1095.

Morice-Picard, F., G. Benard, H. R. Rezvani, E. Lasseaux, D. Simon, S. Moutton, C. Rooryck, D. Lacombe, C. Baumann and B. Arveiler (2016). "Complete loss of function of the ubiquitin ligase HERC2 causes a severe neurodevelopmental phenotype." Eur J Hum Genet 25(1): 52-58. 
Morin, X., R. Daneman, M. Zavortink and W. Chia (2001). "A protein trap strategy to detect GFP-tagged proteins expressed from their endogenous loci in Drosophila." Proc Natl Acad Sci U S A 98(26): 15050-15055.

Nakai, N., M. Nagano, F. Saitow, Y. Watanabe, Y. Kawamura, A. Kawamoto, K. Tamada, H. Mizuma, H. Onoe, Y. Watanabe, H. Monai, H. Hirase, J. Nakatani, H. Inagaki, T. Kawada, T. Miyazaki, M. Watanabe, Y. Sato, S. Okabe, K. Kitamura, M. Kano, K. Hashimoto, H. Suzuki and T. Takumi (2017). "Serotonin rebalances cortical tuning and behavior linked to autism symptoms in 15q11-13 CNV mice." Sci Adv 3(6): e1603001.

Nakatani, J., K. Tamada, F. Hatanaka, S. Ise, H. Ohta, K. Inoue, S. Tomonaga, Y. Watanabe, Y. J. Chung, R. Banerjee, K. Iwamoto, T. Kato, M. Okazawa, K. Yamauchi, K. Tanda, K. Takao, T. Miyakawa, A. Bradley and T. Takumi (2009). "Abnormal behavior in a chromosome-engineered mouse model for human 15q11-13 duplication seen in autism." Cell 137(7): 1235-1246.

Nawaz, Z., D. M. Lonard, C. L. Smith, E. Lev-Lehman, S. Y. Tsai, M. J. Tsai and B. W. O'Malley (1999). "The Angelman syndrome-associated protein, E6-AP, is a coactivator for the nuclear hormone receptor superfamily." Mol Cell Biol 19(2): 1182-1189.

Ng, F. S., S. Sengupta, Y. Huang, A. M. Yu, S. You, M. A. Roberts, L. K. Iyer, Y. Yang and F. R. Jackson (2016). "TRAP-seq Profiling and RNAi-Based Genetic Screens Identify Conserved Glial Genes Required for Adult Drosophila Behavior." Front Mol Neurosci 9: 146.

Noh, H. J., C. P. Ponting, H. C. Boulding, S. Meader, C. Betancur, J. D. Buxbaum, D. Pinto, C. R. Marshall, A. C. Lionel, S. W. Scherer and C. Webber (2013). "Network topologies and convergent aetiologies arising from deletions and duplications observed in individuals with autism." PLoS Genet 9(6): e1003523.

Nuber, U., S. E. Schwarz and M. Scheffner (1998). "The ubiquitin-protein ligase E6associated protein (E6-AP) serves as its own substrate." Eur J Biochem 254(3): 643-649.

Oland, L. A., J. P. Biebelhausen and L. P. Tolbert (2008). "Glial investment of the adult and developing antennal lobe of Drosophila." J Comp Neurol 509(5): 526-550.

Palladino, M. J., J. E. Bower, R. Kreber and B. Ganetzky (2003). "Neural dysfunction and neurodegeneration in Drosophila $\mathrm{Na}+\mathrm{K}+$ ATPase alpha subunit mutants." J Neurosci 23(4): 1276-1286.

Pelc, K., S. G. Boyd, G. Cheron and B. Dan (2008). "Epilepsy in Angelman syndrome." Seizure 17(3): 211-217.

Perea, G., M. Navarrete and A. Araque (2009). "Tripartite synapses: astrocytes process and control synaptic information." Trends Neurosci 32(8): 421-431. 
Pinto, D., A. T. Pagnamenta, L. Klei, R. Anney, D. Merico, R. Regan, J. Conroy, T. R. Magalhaes, C. Correia, B. S. Abrahams, J. Almeida, E. Bacchelli, G. D. Bader, A. J. Bailey, G. Baird, A. Battaglia, T. Berney, N. Bolshakova, S. Bolte, P. F. Bolton, T. Bourgeron, S. Brennan, J. Brian, S. E. Bryson, A. R. Carson, G. Casallo, J. Casey, B. H. Chung, L. Cochrane, C. Corsello, E. L. Crawford, A. Crossett, C. Cytrynbaum, G. Dawson, M. de Jonge, R. Delorme, I. Drmic, E. Duketis, F. Duque, A. Estes, P. Farrar, B. A. Fernandez, S. E. Folstein, E. Fombonne, C. M. Freitag, J. Gilbert, C. Gillberg, J. T. Glessner, J. Goldberg, A. Green, J. Green, S. J. Guter, H. Hakonarson, E. A. Heron, M. Hill, R. Holt, J. L. Howe, G. Hughes, V. Hus, R. Igliozzi, C. Kim, S. M. Klauck, A. Kolevzon, O. Korvatska, V. Kustanovich, C. M. Lajonchere, J. A. Lamb, M. Laskawiec, M. Leboyer, A. Le Couteur, B. L. Leventhal, A. C. Lionel, X. Q. Liu, C. Lord, L. Lotspeich, S. C. Lund, E. Maestrini, W. Mahoney, C. Mantoulan, C. R. Marshall, H. McConachie, C. J. McDougle, J. McGrath, W. M. McMahon, A. Merikangas, O. Migita, N. J. Minshew, G. K. Mirza, J. Munson, S. F. Nelson, C. Noakes, A. Noor, G. Nygren, G. Oliveira, K. Papanikolaou, J. R. Parr, B. Parrini, T. Paton, A. Pickles, M. Pilorge, J. Piven, C. P. Ponting, D. J. Posey, A. Poustka, F. Poustka, A. Prasad, J. Ragoussis, K. Renshaw, J. Rickaby, W. Roberts, K. Roeder, B. Roge, M. L. Rutter, L. J. Bierut, J. P. Rice, J. Salt, K. Sansom, D. Sato, R. Segurado, A. F. Sequeira, L. Senman, N. Shah, V. C. Sheffield, L. Soorya, I. Sousa, O. Stein, N. Sykes, V. Stoppioni, C. Strawbridge, R. Tancredi, K. Tansey, B. Thiruvahindrapduram, A. P. Thompson, S. Thomson, A. Tryfon, J. Tsiantis, H. Van Engeland, J. B. Vincent, F. Volkmar, S. Wallace, K. Wang, Z. Wang, T. H. Wassink, C. Webber, R. Weksberg, K. Wing, K. Wittemeyer, S. Wood, J. Wu, B. L. Yaspan, D. Zurawiecki, L. Zwaigenbaum, J. D. Buxbaum, R. M. Cantor, E. H. Cook, H. Coon, M. L. Cuccaro, B. Devlin, S. Ennis, L. Gallagher, D. H. Geschwind, M. Gill, J. L. Haines, J. Hallmayer, J. Miller, A. P. Monaco, J. I. Nurnberger, Jr., A. D. Paterson, M. A. Pericak-Vance, G. D. Schellenberg, P. Szatmari, A. M. Vicente, V. J. Vieland, E. M. Wijsman, S. W. Scherer, J. S. Sutcliffe and C. Betancur (2010). "Functional impact of global rare copy number variation in autism spectrum disorders." Nature 466(7304): 368372.

Puffenberger, E. G., R. N. Jinks, H. Wang, B. Xin, C. Fiorentini, E. A. Sherman, D. Degrazio, C. Shaw, C. Sougnez, K. Cibulskis, S. Gabriel, R. I. Kelley, D. H. Morton and K. A. Strauss (2012). "A homozygous missense mutation in HERC2 associated with global developmental delay and autism spectrum disorder." Hum Mutat 33(12): 16391646.

Ramirez, J., B. Lectez, N. Osinalde, M. Siva, N. Elu, K. Aloria, M. Prochazkova, C. Perez, J. Martinez-Hernandez, R. Barrio, K. G. Saskova, J. M. Arizmendi and U. Mayor (2018). "Quantitative proteomics reveals neuronal ubiquitination of Rngo/Ddi1 and several proteasomal subunits by Ube3a, accounting for the complexity of Angelman syndrome." Hum Mol Genet 27(11): 1955-1971.

Reichmuth, C., S. Becker, M. Benz, K. Debel, D. Reisch, G. Heimbeck, A. Hofbauer, B. Klagges, G. O. Pflugfelder and E. Buchner (1995). "The sap47 gene of Drosophila melanogaster codes for a novel conserved neuronal protein associated with synaptic terminals." Brain Res Mol Brain Res 32(1): 45-54. 
Reiter, L. T., L. Potocki, S. Chien, M. Gribskov and E. Bier (2001). "A systematic analysis of human disease-associated gene sequences in Drosophila melanogaster." Genome Res 11(6): 1114-1125.

Reiter, L. T., T. N. Seagroves, M. Bowers and E. Bier (2006). "Expression of the RhoGEF Pbl/ECT2 is regulated by the UBE3A E3 ubiquitin ligase." Hum Mol Genet 15(18): 2825-2835.

Renn, S. C., J. H. Park, M. Rosbash, J. C. Hall and P. H. Taghert (1999). "A pdf neuropeptide gene mutation and ablation of PDF neurons each cause severe abnormalities of behavioral circadian rhythms in Drosophila." Cell 99(7): 791-802.

Robinson, W. P., F. Binkert, R. Gine, C. Vazquez, W. Muller, W. Rosenkranz and A. Schinzel (1993). "Clinical and molecular analysis of five inv dup(15) patients." Eur J Hum Genet 1(1): 37-50.

Rodal, A. A., R. N. Motola-Barnes and J. T. Littleton (2008). "Nervous wreck and Cdc42 cooperate to regulate endocytic actin assembly during synaptic growth." J Neurosci 28(33): 8316-8325.

Rougeulle, C., H. Glatt and M. Lalande (1997). "The Angelman syndrome candidate gene, UBE3A/E6-AP, is imprinted in brain." Nat Genet 17(1): 14-15.

Rusan, Z. M., O. A. Kingsford and M. A. Tanouye (2014). "Modeling glial contributions to seizures and epileptogenesis: cation-chloride cotransporters in Drosophila melanogaster." PLoS One 9(6): e101117.

Sailer, C., F. Offensperger, A. Julier, K. M. Kammer, R. Walker-Gray, M. G. Gold, M. Scheffner and F. Stengel (2018). "Structural dynamics of the E6AP/UBE3A-E6-p53 enzyme-substrate complex." Nat Commun 9(1): 4441.

Sanders, S. J., A. G. Ercan-Sencicek, V. Hus, R. Luo, M. T. Murtha, D. Moreno-DeLuca, S. H. Chu, M. P. Moreau, A. R. Gupta, S. A. Thomson, C. E. Mason, K. Bilguvar, P. B. Celestino-Soper, M. Choi, E. L. Crawford, L. Davis, N. R. Wright, R. M. Dhodapkar, M. DiCola, N. M. DiLullo, T. V. Fernandez, V. Fielding-Singh, D. O. Fishman, S. Frahm, R. Garagaloyan, G. S. Goh, S. Kammela, L. Klei, J. K. Lowe, S. C. Lund, A. D. McGrew, K. A. Meyer, W. J. Moffat, J. D. Murdoch, B. J. O'Roak, G. T. Ober, R. S. Pottenger, M. J. Raubeson, Y. Song, Q. Wang, B. L. Yaspan, T. W. Yu, I. R. Yurkiewicz, A. L. Beaudet, R. M. Cantor, M. Curland, D. E. Grice, M. Gunel, R. P. Lifton, S. M. Mane, D. M. Martin, C. A. Shaw, M. Sheldon, J. A. Tischfield, C. A. Walsh, E. M. Morrow, D. H. Ledbetter, E. Fombonne, C. Lord, C. L. Martin, A. I. Brooks, J. S. Sutcliffe, E. H. Cook, Jr., D. Geschwind, K. Roeder, B. Devlin and M. W. State (2011). "Multiple recurrent de novo CNVs, including duplications of the 7q11.23 Williams syndrome region, are strongly associated with autism." Neuron 70(5): 863-885.

Sandin, S., P. Lichtenstein, R. Kuja-Halkola, C. Hultman, H. Larsson and A. Reichenberg (2017). "The Heritability of Autism Spectrum Disorder." JAMA 318(12): 1182-1184. 
Sato, M. (2017). "Early Origin and Evolution of the Angelman Syndrome Ubiquitin Ligase Gene Ube3a." Front Cell Neurosci 11: 62.

Sau, A., F. Pellizzari Tregno, F. Valentino, G. Federici and A. M. Caccuri (2010). "Glutathione transferases and development of new principles to overcome drug resistance." Arch Biochem Biophys 500(2): 116-122.

Saumweber, T., A. Weyhersmuller, S. Hallermann, S. Diegelmann, B. Michels, D. Bucher, N. Funk, D. Reisch, G. Krohne, S. Wegener, E. Buchner and B. Gerber (2011). "Behavioral and synaptic plasticity are impaired upon lack of the synaptic protein SAP47." J Neurosci 31(9): 3508-3518.

Scharfman, H. E. (2007). "The neurobiology of epilepsy." Curr Neurol Neurosci Rep 7(4): 348-354.

Scheffner, M., J. M. Huibregtse, R. D. Vierstra and P. M. Howley (1993). "The HPV-16 E6 and E6-AP complex functions as a ubiquitin-protein ligase in the ubiquitination of p53." Cell 75(3): 495-505.

Scheffner, M. and S. Kumar (2014). "Mammalian HECT ubiquitin-protein ligases: biological and pathophysiological aspects." Biochim Biophys Acta 1843(1): 61-74.

Schmid, B., C. Helfrich-Forster and T. Yoshii (2011). "A new ImageJ plug-in "ActogramJ" for chronobiological analyses." J Biol Rhythms 26(5): 464-467.

Schneider, C. A., W. S. Rasband and K. W. Eliceiri (2012). "NIH Image to ImageJ: 25 years of image analysis." Nat Methods 9(7): 671-675.

Schroer, R. J., M. C. Phelan, R. C. Michaelis, E. C. Crawford, S. A. Skinner, M. Cuccaro, R. J. Simensen, J. Bishop, C. Skinner, D. Fender and R. E. Stevenson (1998). "Autism and maternally derived aberrations of chromosome 15q." Am J Med Genet 76(4): 327 336.

Schubert, J., A. Siekierska, M. Langlois, P. May, C. Huneau, F. Becker, H. Muhle, A. Suls, J. R. Lemke, C. G. de Kovel, H. Thiele, K. Konrad, A. Kawalia, M. R. Toliat, T. Sander, F. Ruschendorf, A. Caliebe, I. Nagel, B. Kohl, A. Kecskes, M. Jacmin, K. Hardies, S. Weckhuysen, E. Riesch, T. Dorn, E. H. Brilstra, S. Baulac, R. S. Moller, H. Hjalgrim, B. P. Koeleman, E. R. E. S. C. Euro, K. Jurkat-Rott, F. Lehman-Horn, J. C. Roach, G. Glusman, L. Hood, D. J. Galas, B. Martin, P. A. de Witte, S. Biskup, P. De Jonghe, I. Helbig, R. Balling, P. Nurnberg, A. D. Crawford, C. V. Esguerra, Y. G. Weber and H. Lerche (2014). "Mutations in STX1B, encoding a presynaptic protein, cause fever-associated epilepsy syndromes." Nat Genet 46(12): 1327-1332.

Schubiger, M., Y. Feng, D. M. Fambrough and J. Palka (1994). "A mutation of the Drosophila sodium pump alpha subunit gene results in bang-sensitive paralysis." Neuron 12(2): 373-381. 
Schulze, K. L., K. Broadie, M. S. Perin and H. J. Bellen (1995). "Genetic and electrophysiological studies of Drosophila syntaxin-1A demonstrate its role in nonneuronal secretion and neurotransmission." Cell 80(2): 311-320.

Scoles, H. A., N. Urraca, S. W. Chadwick, L. T. Reiter and J. M. Lasalle (2011). "Increased copy number for methylated maternal 15q duplications leads to changes in gene and protein expression in human cortical samples." Mol Autism 2(1): 19.

Sebat, J., B. Lakshmi, D. Malhotra, J. Troge, C. Lese-Martin, T. Walsh, B. Yamrom, S. Yoon, A. Krasnitz, J. Kendall, A. Leotta, D. Pai, R. Zhang, Y. H. Lee, J. Hicks, S. J. Spence, A. T. Lee, K. Puura, T. Lehtimaki, D. Ledbetter, P. K. Gregersen, J. Bregman, J. S. Sutcliffe, V. Jobanputra, W. Chung, D. Warburton, M. C. King, D. Skuse, D. H. Geschwind, T. C. Gilliam, K. Ye and M. Wigler (2007). "Strong association of de novo copy number mutations with autism." Science 316(5823): 445-449.

Seifert, G. and C. Steinhauser (2013). "Neuron-astrocyte signaling and epilepsy." Exp Neurol 244: 4-10.

Shaaya, E. A., O. R. Grocott, O. Laing and R. L. Thibert (2016). "Seizure treatment in Angelman syndrome: A case series from the Angelman Syndrome Clinic at Massachusetts General Hospital." Epilepsy Behav 60: 138-141.

Shang, W., W. H. Liu, X. H. Zhao, Q. J. Sun, J. Z. Bi and Z. F. Chi (2008). "Expressions of glutathione S-transferase alpha, mu, and pi in brains of medically intractable epileptic patients." BMC Neurosci 9: 67.

Shi, S. Q., T. J. Bichell, R. A. Ihrie and C. H. Johnson (2015). "Ube3a imprinting impairs circadian robustness in Angelman syndrome models." Curr Biol 25(5): 537-545.

Shih, J., R. Hodge and M. A. Andrade-Navarro (2015). "Comparison of inter- and intraspecies variation in humans and fruit flies." Genom Data 3: 49-54.

Shishido, E., B. Aleksic and N. Ozaki (2014). "Copy-number variation in the pathogenesis of autism spectrum disorder." Psychiatry Clin Neurosci 68(2): 85-95.

Sievers, F., A. Wilm, D. Dineen, T. J. Gibson, K. Karplus, W. Li, R. Lopez, H. McWilliam, M. Remmert, J. Soding, J. D. Thompson and D. G. Higgins (2011). "Fast, scalable generation of high-quality protein multiple sequence alignments using Clustal Omega." Mol Syst Biol 7: 539.

Silva-Santos, S., G. M. van Woerden, C. F. Bruinsma, E. Mientjes, M. A. Jolfaei, B. Distel, S. A. Kushner and Y. Elgersma (2015). "Ube3a reinstatement identifies distinct developmental windows in a murine Angelman syndrome model." J Clin Invest 125(5): 2069-2076.

Simon, A. F., M. T. Chou, E. D. Salazar, T. Nicholson, N. Saini, S. Metchev and D. E. Krantz (2012). "A simple assay to study social behavior in Drosophila: measurement of social space within a group." Genes Brain Behav 11(2): 243-252. 
Sinakevitch, I., Y. Grau, N. J. Strausfeld and S. Birman (2010). "Dynamics of glutamatergic signaling in the mushroom body of young adult Drosophila." Neural Dev 5: 10 .

Smith, S. E., Y. D. Zhou, G. Zhang, Z. Jin, D. C. Stoppel and M. P. Anderson (2011). "Increased gene dosage of Ube3a results in autism traits and decreased glutamate synaptic transmission in mice." Sci Transl Med 3(103): 103 ra197.

Somjen, G. G. (2002). "Ion regulation in the brain: implications for pathophysiology." Neuroscientist 8(3): 254-267.

Spadaro, M., S. Ursu, F. Lehmann-Horn, L. Veneziano, G. Antonini, P. Giunti, M. Frontali and K. Jurkat-Rott (2004). "A G301R Na+/K+ -ATPase mutation causes familial hemiplegic migraine type 2 with cerebellar signs." Neurogenetics 5(3): 177-185.

Stiles, J. and T. L. Jernigan (2010). "The basics of brain development." Neuropsychol Rev 20(4): 327-348.

Stone, B., B. Burke, J. Pathakamuri, J. Coleman and D. Kuebler (2014). "A low-cost method for analyzing seizure-like activity and movement in Drosophila." J Vis Exp (84): e51460.

Sun, B., P. Xu, W. Wang and P. M. Salvaterra (2001). "In vivo modification of $\mathrm{Na}(+), \mathrm{K}(+)$-ATPase activity in Drosophila." Comp Biochem Physiol B Biochem Mol Biol 130(4): 521-536.

Swoboda, K. J., E. Kanavakis, A. Xaidara, J. E. Johnson, M. F. Leppert, M. B. Schlesinger-Massart, L. J. Ptacek, K. Silver and S. Youroukos (2004). "Alternating hemiplegia of childhood or familial hemiplegic migraine? A novel ATP1A2 mutation." Ann Neurol 55(6): 884-887.

Sykiotis, G. P. and D. Bohmann (2008). "Keap1/Nrf2 signaling regulates oxidative stress tolerance and lifespan in Drosophila." Dev Cell 14(1): 76-85.

Tew, K. D., S. Dutta and M. Schultz (1997). "Inhibitors of glutathione S-transferases as therapeutic agents." Adv Drug Deliv Rev 26(2-3): 91-104.

Thibert, R. L., K. D. Conant, E. K. Braun, P. Bruno, R. R. Said, M. P. Nespeca and E. A. Thiele (2009). "Epilepsy in Angelman syndrome: a questionnaire-based assessment of the natural history and current treatment options." Epilepsia 50(11): 2369-2376.

Townsend, D. M. and K. D. Tew (2003). "The role of glutathione-S-transferase in anticancer drug resistance." Oncogene 22(47): 7369-7375.

Ugur, B., K. Chen and H. J. Bellen (2016). "Drosophila tools and assays for the study of human diseases." Dis Model Mech 9(3): 235-244. 
Urraca, N., J. Cleary, V. Brewer, E. K. Pivnick, K. McVicar, R. L. Thibert, N. C. Schanen, C. Esmer, D. Lamport and L. T. Reiter (2013). "The interstitial duplication 15q11.2-q13 syndrome includes autism, mild facial anomalies and a characteristic EEG signature." Autism Res 6(4): 268-279.

Urraca, N., K. Hope, A. K. Victor, T. G. Belgard, R. Memon, S. Goorha, C. Valdez, Q. T. Tran, S. Sanchez, J. Ramirez, M. Donaldson, D. Bridges and L. T. Reiter (2018). "Significant transcriptional changes in 15q duplication but not Angelman syndrome deletion stem cell-derived neurons." Mol Autism 9: 6.

Valdez, C., R. Scroggs, R. Chassen and L. T. Reiter (2015). "Variation in Dube3a expression affects neurotransmission at the Drosophila neuromuscular junction." Biol Open 4(7): 776-782.

Vanmolkot, K. R., E. E. Kors, J. J. Hottenga, G. M. Terwindt, J. Haan, W. A. Hoefnagels, D. F. Black, L. A. Sandkuijl, R. R. Frants, M. D. Ferrari and A. M. van den Maagdenberg (2003). "Novel mutations in the $\mathrm{Na}+, \mathrm{K}+$-ATPase pump gene ATP1A2 associated with familial hemiplegic migraine and benign familial infantile convulsions." Ann Neurol 54(3): 360-366.

Vardjan, N., V. Parpura and R. Zorec (2016). "Loose excitation-secretion coupling in astrocytes." Glia 64(5): 655-667.

Vilinsky, I. and K. G. Johnson (2012). "Electroretinograms in Drosophila: a robust and genetically accessible electrophysiological system for the undergraduate laboratory." J Undergrad Neurosci Educ 11(1): A149-157.

Wagh, D. A., T. M. Rasse, E. Asan, A. Hofbauer, I. Schwenkert, H. Durrbeck, S. Buchner, M. C. Dabauvalle, M. Schmidt, G. Qin, C. Wichmann, R. Kittel, S. J. Sigrist and E. Buchner (2006). "Bruchpilot, a protein with homology to ELKS/CAST, is required for structural integrity and function of synaptic active zones in Drosophila." Neuron 49(6): 833-844.

Wang, L., K. J. Colodner and M. B. Feany (2011). "Protein misfolding and oxidative stress promote glial-mediated neurodegeneration in an Alexander disease model." $\underline{\mathrm{J}}$ Neurosci 31(8): 2868-2877.

Wang, L., T. L. Hagemann, A. Messing and M. B. Feany (2016). "An In Vivo Pharmacological Screen Identifies Cholinergic Signaling as a Therapeutic Target in Glial-Based Nervous System Disease." J Neurosci 36(5): 1445-1455.

Wegiel, J., N. C. Schanen, E. H. Cook, M. Sigman, W. T. Brown, I. Kuchna, K. Nowicki, J. Wegiel, H. Imaki, S. Y. Ma, E. Marchi, T. Wierzba-Bobrowicz, A. Chauhan, V. Chauhan, I. L. Cohen, E. London, M. Flory, B. Lach and T. Wisniewski (2012). "Differences between the pattern of developmental abnormalities in autism associated with duplications 15q11.2-q13 and idiopathic autism." J Neuropathol Exp Neurol 71(5): 382-397. 
Weiss, L. A., Y. P. Shen, J. M. Korn, D. E. Arking, D. T. Miller, R. Fossdal, E. Saemundsen, H. Stefansson, M. A. R. Ferreira, T. Green, O. S. Platt, D. M. Ruderfer, C. A. Walsh, D. Altshuler, A. Chakravarti, R. E. Tanzi, K. Stefansson, S. L. Santangelo, J. F. Gusella, P. Sklar, B. Wu, M. J. Daly and A. Consortium (2008). "Association between microdeletion and microduplication at 16p11.2 and autism." New England Journal of Medicine 358(7): 667-675.

Wetherington, J., G. Serrano and R. Dingledine (2008). "Astrocytes in the epileptic brain." Neuron 58(2): 168-178.

Williams, C. A., A. L. Beaudet, J. Clayton-Smith, J. H. Knoll, M. Kyllerman, L. A. Laan, R. E. Magenis, A. Moncla, A. A. Schinzel, J. A. Summers and J. Wagstaff (2006). "Angelman syndrome 2005: updated consensus for diagnostic criteria." Am J Med Genet A 140(5): 413-418.

Winther, A. M., O. Vorontsova, K. A. Rees, T. Nareoja, E. Sopova, W. Jiao and O. Shupliakov (2015). "An Endocytic Scaffolding Protein together with Synapsin Regulates Synaptic Vesicle Clustering in the Drosophila Neuromuscular Junction." J Neurosci 35(44): 14756-14770.

Wu, Y., F. V. Bolduc, K. Bell, T. Tully, Y. Fang, A. Sehgal and J. A. Fischer (2008). "A Drosophila model for Angelman syndrome." Proc Natl Acad Sci U S A 105(34): 1239912404.

Wu, Y., D. Liu and Z. Song (2015). "Neuronal networks and energy bursts in epilepsy." Neuroscience 287: 175-186.

Xiong, W. C., H. Okano, N. H. Patel, J. A. Blendy and C. Montell (1994). "repo encodes a glial-specific homeo domain protein required in the Drosophila nervous system." Genes Dev 8(8): 981-994.

Yamasaki, K., K. Joh, T. Ohta, H. Masuzaki, T. Ishimaru, T. Mukai, N. Niikawa, M. Ogawa, J. Wagstaff and T. Kishino (2003). "Neurons but not glial cells show reciprocal imprinting of sense and antisense transcripts of Ube3a." Hum Mol Genet 12(8): 837-847.

Yoo, H. (2015). "Genetics of Autism Spectrum Disorder: Current Status and Possible Clinical Applications." Exp Neurobiol 24(4): 257-272.

Zheng, N. and N. Shabek (2017). "Ubiquitin Ligases: Structure, Function, and Regulation." Annu Rev Biochem. 


\section{VITA}

Kevin Hope was born in Portland, Oregon in 1991 and grew up on Bainbridge Island, Washington. He completed his undergraduate degree at Western Washington University and received his bachelor's degree in Behavioral Neuroscience in 2013. In 2013 he moved to Memphis, Tennessee and attended The University of Tennessee Health Science Center where he worked on attaining his Ph.D in the College of Graduate Health Sciences Integrated Program in Biomedical Sciences, Neuroscience Track with Dr. Lawrence Reiter. After graduation he plans to pursue a Postdoctoral Scholar position with Dr. Clement Chow at The University of Utah. 